University of Louisville

ThinkIR: The University of Louisville's Institutional Repository

Electronic Theses and Dissertations

$5-2015$

\title{
Glycine receptor alpha subunit (GlyRa) specific inhibition contributes to ganglion cell signaling in mouse retina.
}

Chi Zhang

University of Louisville

Follow this and additional works at: https://ir.library.louisville.edu/etd

Part of the Anatomy Commons, and the Neuroscience and Neurobiology Commons

\section{Recommended Citation}

Zhang, Chi, "Glycine receptor alpha subunit (GlyRa) specific inhibition contributes to ganglion cell signaling in mouse retina." (2015). Electronic Theses and Dissertations. Paper 2075.

https://doi.org/10.18297/etd/2075

This Doctoral Dissertation is brought to you for free and open access by ThinkIR: The University of Louisville's Institutional Repository. It has been accepted for inclusion in Electronic Theses and Dissertations by an authorized administrator of ThinkIR: The University of Louisville's Institutional Repository. This title appears here courtesy of the author, who has retained all other copyrights. For more information, please contact thinkir@louisville.edu. 
GLYCINE RECEPTOR ALPHA SUBUNIT (GlyRa) SPECIFIC INHIBITION CONTRIBUTES TO GANGLION CELL SIGNALING IN MOUSE RETINA

By

\author{
Chi Zhang \\ B.S., Xiamen University, 2006 \\ A Dissertation \\ Submitted to the Faculty of the \\ School of Medicine of the University of Louisville \\ in Partial Fulfillment of the Requirements \\ for the Degree of
}

Doctor of Philosophy in Anatomical Sciences and Neurobiology

Department of Anatomical Sciences and Neurobiology

University of Louisville

Louisville, Kentucky

May 2015 

GLYCINE RECEPTOR ALPHA SUBUNIT (GlyRa) SPECIFIC INHIBITION CONTRIBUTES TO GANGLION CELL SIGNALING IN MOUSE RETINA

By

Chi Zhang

B.S., Xiamen University, 2006

A Dissertation Approved on

March 12, 2015

by the following Dissertation Committee:

Maureen McCall, Ph.D.

Martha Bickford, Ph.D.

Bart Borghuis, Ph.D.

Ronald Gregg, Ph.D.

Robin Krimm, Ph.D.

Zhenmin Lei, M.D., Ph.D. 


\section{ACKNOWLEDGMENTS}

The first step of everything is always difficult, yet I believe it is more difficult to successfully accomplish what has been started. I owe a lot of gratitude to many people for their help which carry me through my graduate career and complete my work. I would like to thank my parents, Mr. Zhijian Zhang and Mrs. Jing Zhou, for their love and care ever since I came to this world. I'm grateful for their continuous support for my academic pursuits. I cherish very much the beautiful friendships built up along this six-and-a-half-year journey with many people in graduate school, especially Penny Cheng and Yanfang Peipei Zhu, who have been there with me through all the ups and downs. I have been very fortunate to have many excellent teachers. Dr. Robert Lundy helped me develop my interests and ability in scientific research in neuroscience when I started graduate school. All my committee members, Drs. Martha Bickford, Bart Borghuis, Nigel Cooper, Ronald Gregg, Robin Krimm and Zhenmin Lei, have been generously offering their constructive advices and invaluable help on my graduate study. Dr. Botond Roska made my graduate career fruitful and exciting by taking me to experience cutting-edge scientific research in his lab. Lastly, I could not have made it so far without my mentor, Dr. Maureen McCall. With the years of working with her, I have learned not only the necessary research skills, but more importantly the right attitude to be a good scientist, which is to always 
be passionate about science and positive to challenges. I am truly thankful for all these great things she has taught me. 


\begin{abstract}
GLYCINE RECEPTOR ALPHA SUBUNIT (GlyRa) SPECIFIC INHIBITION CONTRIBUTES TO GANGLION CELL SIGNALING IN MOUSE RETINA
\end{abstract}

Chi Zhang

March 12, 2015

In the retina, numerous types of neurons are wired together in a highly specific albeit complex pattern. This sophisticated retinal network allows extraction and encoding of more than 20 representations of the visual scene in its output neurons, the retinal ganglion cells (RGCs). Within the inner plexiform layer (IPL) of retina, glycine receptors (GlyRs) are expressed on different cell classes and modulate RGC visual activity to light onset (ON RGCs) and to light offset (OFF RGCs), for example, their temporal precision and gain control. There are four GlyR alpha subunits (GlyRa1-4) with differential expression patterns in IPL. Each mediates spontaneous inhibitory postsynaptic currents (sIPSCs) with different decay kinetics. Moreover, GlyR alpha subunit-specific expression was discovered across different RGC types. This evidence suggests subunit-specific roles for glycinergic inhibitory circuits to modulate the RGC visual outputs. However, the details remain largely unknown. 
To investigate glycinergic subunit-specific modulation, I used GlyRa subunit knockout (KO) mouse lines, which lack GlyRa2 (Glra2 $\left.{ }^{-/}\right)$, GlyRa3 (Glra3 $\left.{ }^{-/}\right)$ or both $($ Glra2/3/- $)$. I found that GlyRa2 and GlyRa3 enhance ON RGCs visual responses whereas only GlyRa2 enhances OFF RGCs visual responses. Second, I used viral tools to manipulate the expression of the GlyRa1 subunit on RGCs to examine its role in visual processing. Adeno-associated viruses (AAVs) were injected into dorsal lateral geniculate nucleus and transported retrogradely to infect RGCs and generate shRNA to selectively knockdown GlyRa1 expression. In OFFa $\alpha_{\text {Transient }} R G C s$, which predominantly express GlyRa1, shRNA almost completely eliminated all glycinergic input and I showed that this input increases signal to noise ratio of OFF $\alpha_{\text {Transient }} R G C$ visual responses. I expanded our understanding of subunit-specificity by surveying subunit specific expression and currents across eight identified RGCs in the $P V^{\text {cre }}$ mouse. By comparing colocalization of GlyR a subunit puncta on identified RGC dendrites with the decay kinetics of their sIPSCs, I showed that there is subunit-specific expression of GlyRs.

My data not only support the hypothesis of subunit-specific glycinergic inhibitory modulation in retinal signaling, but provide new tools to further explore their individual roles in shaping RGC visual function. 


\section{TABLE OF CONTENTS}

PAGE

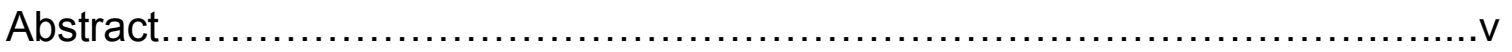

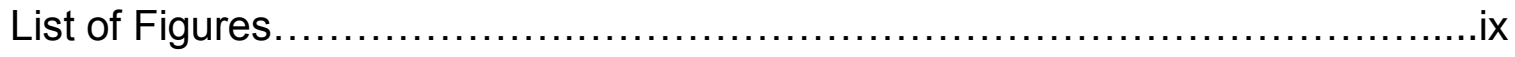

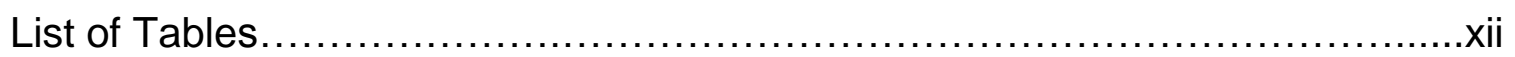

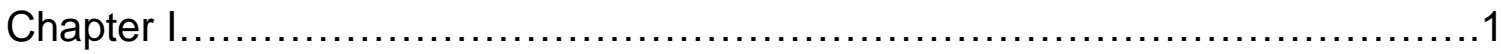

Introduction

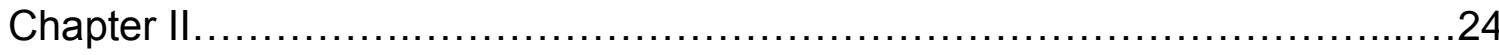

Experimental Materials and Methods

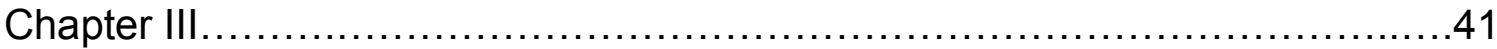

The role of GlyRa2- and GlyRa3-mediated Inhibition in the Receptive Field Surround in Retinal Ganglion Cells

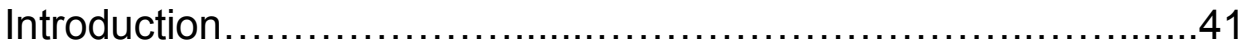

Results.......................................................... 47

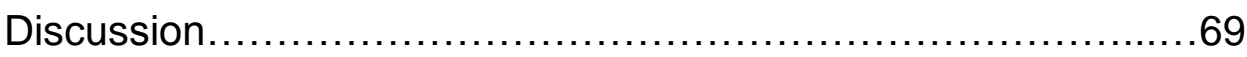

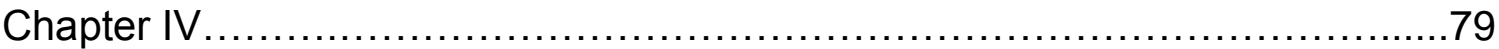

Adeno-Associated Virus-RNAi of GlyRa1 and Characterization of Its

Synapse-specific Inhibition in OFF alpha Transient Retinal Ganglion Cells

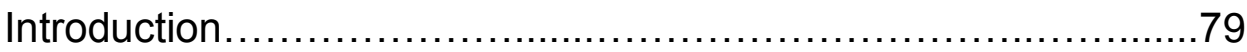

Results.............................................................. 82

Discussion........................................................ 102

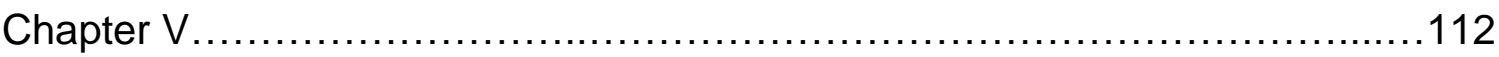

Subunit Specific Expression of Glycine Receptor in Mouse Retinal

Ganglion Cells 
Introduction

Results.

Discussion

Chapter VI.

General Discussion and Future Directions

References......

Curriculum Vitae.
112

115

140

149

157

168 


\section{LIST OF FIGURES}

FIGURE

PAGE

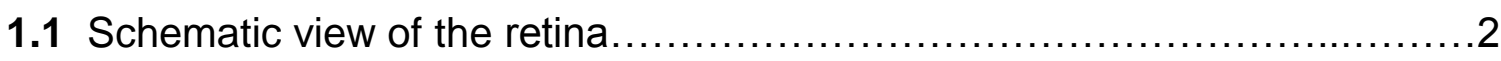

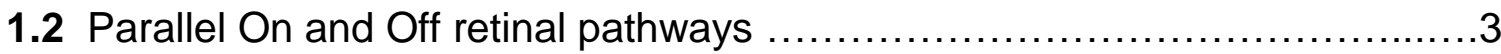

1.3 Three rod signaling pathways in the mammalian retina....................5

1.4 ON and OFF RGCs RF center summation...............................

1.5 Pentameric structure of ionotropic inhibitory receptors and their subunits...12

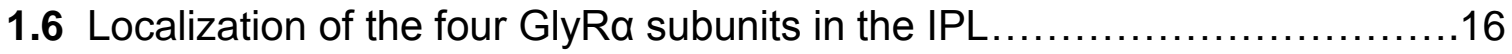

1.7 Vertical sections of a mouse retina double labeled for GlyRa4 and ChAT...16

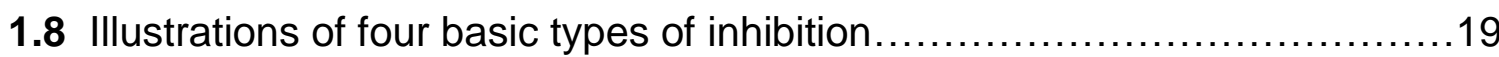

2.1 PSTH response quantification during receptive field surround stimulation...29

2.2 A schematic diagram of the AAV-RNAi vector backbone, pAAV-Ef1a-

NLStdTomato-H1

3.1 Vertical cross sections of mouse retina labeled for the four GlyR a

subunits.

3.2 Representative PSTHs from an WT ON sustained RGC in the presence of

bright annulus with varying inner diameter....

3.3 RF optimal surround suppressive responses of WT ON and OFF RGCs...53

3.4 RF optimal surround suppressive responses of GlyR KO ON and OFF

RGCs 
3.5 GlyRa2- and GlyRa3 does not contribute to the receptive field surround suppression of ON RGCs

3.6 Only GlyRa2-mediated inhibition modulates the receptive field suppression of OFF RGCs .58

3.7 The post-stimulus peak amplitude of the OFF RGC is affected by the absence of GlyRa2 expression.

3.8 A Difference of Gaussians model for RF center/surround spatial organization 64

3.9 A model of altered balanced-response inner diameter of ON but not OFF RGCs.

3.10 The absence of GlyRa2 or GlyRa3 alters the balanced-response inner diameter of ON but not OFF RGCs

3.11 Schematic diagrams of the proposed roles of GlyRa2 in the WT Off mouse retinal pathways .75

4.1 The GlyRa1 subunit is expressed on PV $5_{W T} R G C$ dendrites.

4.2 GlyRa1-meditated sIPSC and glycinergic elPSCs are present in PV5WT RGCs. 86

4.3 AAV-mediated RNAi significantly knockdowns GlyRa1 expression on PV5 RGC dendrites 88

4.4 Knockdown of Glra1 eliminates GlyRa1 sIPSCs in PV5 RGCs.

4.5 Knockdown of Glra1 eliminates GlyRa1 input to PV5 RGCs at luminance increments. 
4.6 GlyRa1 input modulates PV5 RGC spontaneous activity and the excitatory rebound post stimulus suppression to a luminance increment 96

4.7 Stimulus evoked responses to luminance decrement is modulated by glycinergic and $\mathrm{GABA}_{A}$ ergic inputs 98

4.8 The light evoked responses of PV5 ${ }_{W T}$ RGCs are modulated by both GlyRa1 and $\mathrm{GABA}_{\mathrm{A}}$ ergic inputs 107

5.1 Summary of morphological quantification of $P V^{\text {cre }}$ RGCs..................116

5.2 The GlyRa1 subunit is expressed on PV1, PV6 and PV2 RGCs. 118

5.3 PV1, 2, 5 and 6 RGCs receive GlyRa1-mediated spontaneous inhibitory postsynaptic currents 121

5.4 PV4 RGCs significantly express GlyRa1 subunits on their dendrites.......125

5.5 PV4 receive sIPSCs that are mediated both by GlyRa1 and GlyRa3..... 126

5.6 PV7 RGCs significantly express GlyRa3 subunits on their dendrites........129

5.7 PV7 receive sIPSCs mediated by GlyRa3 and GlyRa2 ..................132

5.8 The morphology of PVO and TRHR RGCs and expression of different GlyRa subunits on PVO dendrites. 135

5.9 PV0 and TRHR RGCs receive sIPSC with slow decay constant. 139

5.10 Cumulative frequency distribution of $T_{\text {decay }}$ of glycinergic sIPSCs in PV RGCs. 147 


\section{LIST OF TABLES}

TABLE

PAGE

1.1 Parameters for GABAergic sIPSCs and elPSCs from ON cone BCs ........14

1.2 Different cell types express specific GlyRa subunits with distinct kinetics...17

2.1 List of mouse lines used for experiments .................................25

3.1 Numbers of RGC with any surround suppression across genotypes .........48

3.2 Summary of ON RGCs with three different maintained response profiles

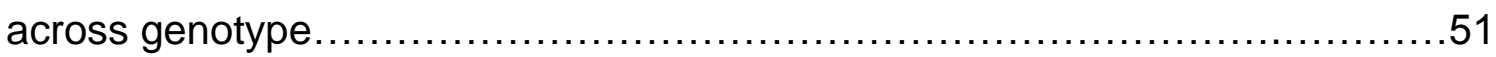

3.3 Summary of OFF RGCs with sustained and transient maintained response

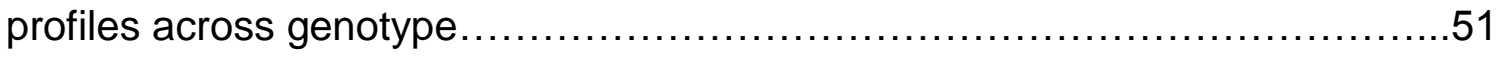

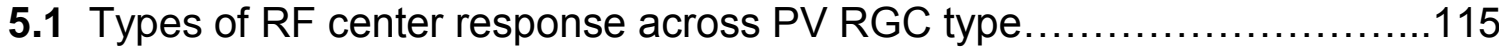

5.2 Summary of GlyR subunit-specific sIPSC properties in PV RGCs.........142 


\section{CHAPTER I}

\section{INTRODUCTION}

\section{Retinal Cell Classes and Basic Circuitry}

\subsection{Retinal Laminar Structure and Their Cell Classes}

The retina is a laminar structure that is a part of the central nervous system. It is organized into three nuclear and two synaptic layers and five cell classes are selectively distributed across these layers (Figure 1.1). The outer nuclear layer (ONL) contains the cell bodies of the photoreceptors (PRs), the cones and rods. The inner nuclear layer (INL) contains the cell bodies of bipolar (BCs), horizontal (HCs) and amacrine cells (ACs). The ganglion cell layer (GCL) contains the cell bodies of ganglion cells (RGCs) and displaced ACs.

The visual signal arises in the PRs and is relayed via synapses in the outer plexiform layer (OPL) to the BCs and HCs. The output of processing in the ONL is transmitted to the RGCs via synapses in the inner plexiform layer (IPL). The excitatory signal is transmitted vertically from the PRs through the BCs to RGCs. These excitatory processes are modulated by lateral inhibitory mechanisms that involve the HCs and ACs within OPL and IPL, respectively. This modulation shapes both spatial and temporal aspects of the signal. Throughout most of the retina, processing occurs via analog signaling and slow 
potentials. At the level of some of the ACs and all of the RGCs the signal is converted to digital in the form of spiking activity, which forms the output to the rest of the brain.

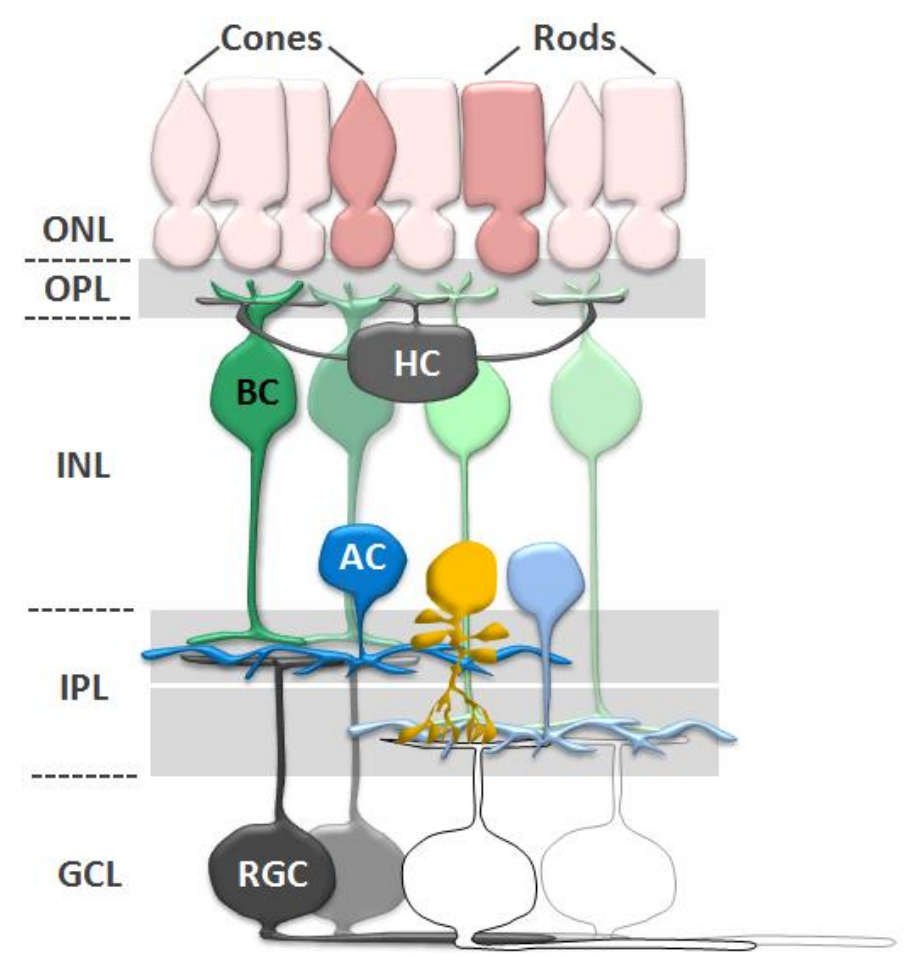

Figure 1.1. Schematic view of the retina. Two types of photoreceptors (rods and cones), horizontal cells (HCs), bipolar cells (BCs), amacrine cells (ACs) and retinal ganglion cells (RGCs) are arranged in a laminar fashion within the retina. Light signals arriving at rods and cones are transmitted across all the layers from $B C$ s to RGCs. HCs and ACs act to provide lateral information (ONL, outer nuclear layer; OPL, outer plexiform layer; INL, inner nuclear layer; IPL, inner plexiform layer; GCL, ganglion cell layer). 


\subsection{Parallel Pathways Are a Basic Feature of Retinal Signaling}

The retina operates over a very wide range of lighting conditions $\left(10^{-6}\right.$ to $\left.10^{6} \mathrm{~cd} / \mathrm{m}^{2}\right)$. To accomplish this versatility, the retina utilizes parallel On and Off pathways to encode luminance increments and decrements (Figure 1.2).

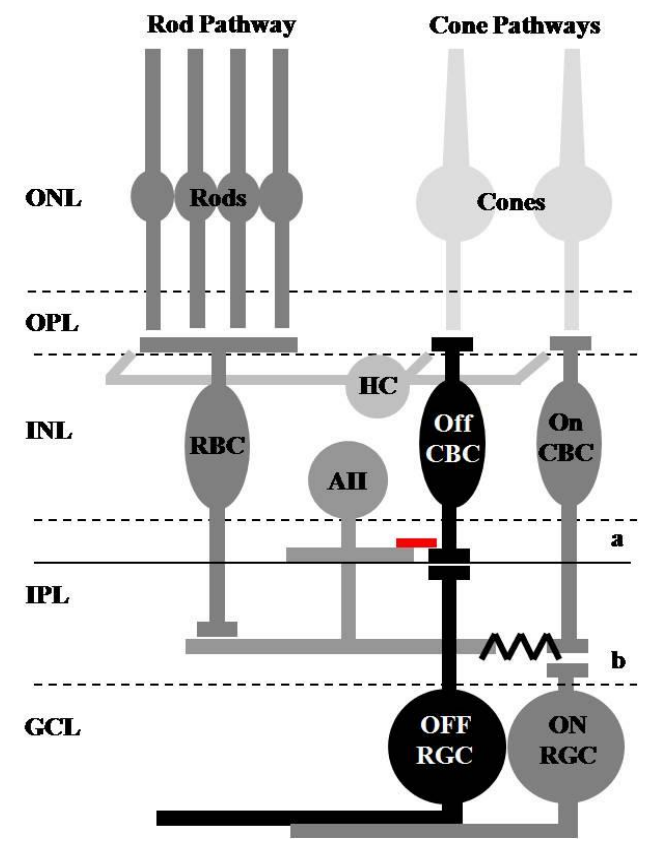

Figure 1.2. Parallel On and Off retinal pathways. Cones contact ON and OFF cone BCs (CBCs), which in turn directly synapse upon their corresponding ON or OFF RGCs. Multiple rods contact a single rod BC (RBC), which uses the All AC as interneuron to relay information to the parallel ON and OFF cone pathways. The All uses a sign-inverting glycinergic synapse ( $($ ) to transmit information to the OFF CBCs and OFF RGCs, and a sign-conserving electrical synapse (M) to pass information to the ON CBCs and ON RGCs. 
Rods are sensitive to very low luminance levels and are responsible for scotopic vision $\left(10^{-6}\right.$ to $\left.10^{-2} \mathrm{~cd} / \mathrm{m}^{2}\right)$. They are so sensitive that they can signal a single photon (Hecht et al., 1941). Cones are less sensitive and are responsible for photopic vision (1 to $\left.10^{6} \mathrm{~cd} / \mathrm{m}^{2}\right)$.

\subsubsection{The Depolarizing (ON) and Hyperpolarizing (OFF) Cone Pathways}

Cones synapse onto two different general types of BCs and establish separate pathways to signal luminance increments (ON) and decrements (OFF). Depolarizing BCs (both rod and cone DBCs) express the metabotropic glutamate receptor 6 (mGluR6 receptor or GRM6 protein) on their dendrites in the OPL (Masu et al., 1995). In the dark, PRs (rods and cones) constantly release glutamate, which keeps the mGluR6 receptor occupied and inactivates its Gprotein coupled cascade. This keeps the TRPM1 cation channel closed and DBCs relatively hyperpolarized (Koike et al., 2010; Morgans et al., 2009; Snellman et al., 2008). At light onset, PRs reduce glutamate release, which activates the mGluR6 G-protein signaling cascade, opens the TRPM1 cation channel and depolarizes the DBC. Hyperpolarizing BCs (only cone HBCs) express ionotropic glutamate receptors on their dendrites. Currently conflicting results indicate that either AMPA and Kainate (DeVries, 2000; Puller et al., 2013) or Kainate alone (Borghuis et al., 2014) are responsible for signaling. In contrast to the ON pathway, the binding of glutamate onto ionotropic glutamate receptors keeps the HBCs relatively depolarized in the dark. At light offset, when glutamate levels increase the HBC is depolarized (Kaneko and Saito, 1983; Saito and Kaneko, 1983). In mouse retina, there are five types of DBCs and four types of 
HBCs. They are differentiated by their morphology and by the termination patterns of their axons at that define the On and Off sublaminae of the IPL (Ghosh et al., 2004). DBC axons terminate and make glutamatergic synapses with ON ACs and RGCs within the On sublaminae. HBC axons terminate and make glutamatergic synapses with OFF ACs and RGCs within the Off sublaminae. The glutamatergic BC-to-RGC inputs represent vertical excitatory transmission, whereas $\mathrm{BC}$-to-AC inputs drive diverse lateral inhibitory pathways and modulate the outputs of BCs and RGCs.

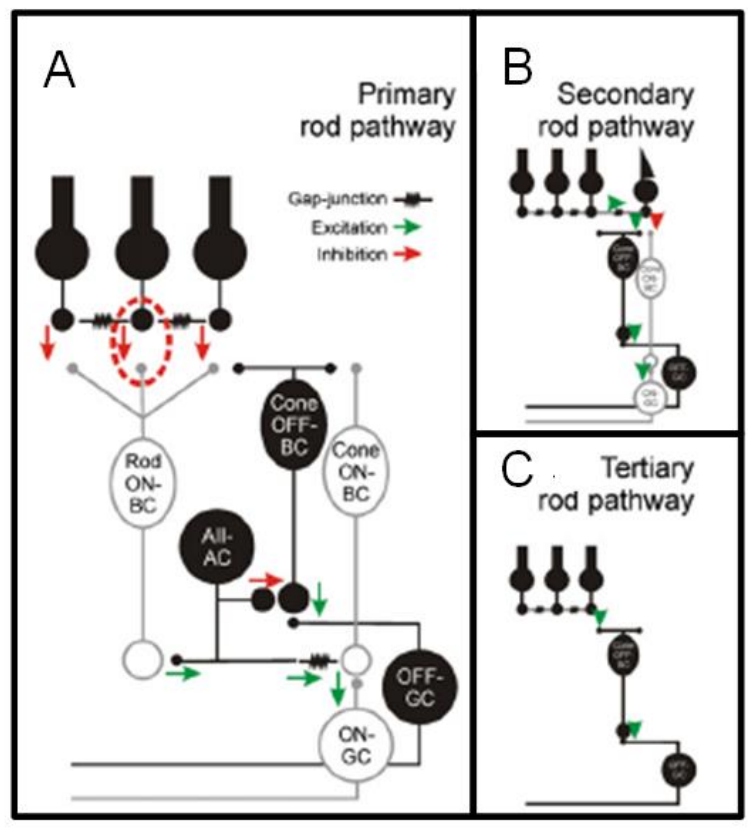

Figure 1.3. Three rod signaling pathways in the mammalian retina. (A) The primary rod pathway. Rods drive rod ON BCs to relay visual information to All $\mathrm{AC}$, which are electrically coupled with $\mathrm{ON}$ cone BCs and inhibit OFF cone BCs via a glycinergic synapse. (B) The secondary rod pathway. Rods are electrically coupled to cones and allow the rod signal into ON and OFF cone BC pathways to 
the RGCs. (C) The tertiary rod pathway. Rods provide a direct glutamatergic input to OFF cone BCs. (modified from van Genderen et al., 2009)

\subsubsection{The Rod Pathways}

Compared to the cone pathway, the rod pathway is more specialized. Rods synapse almost exclusively with rod DBCs, which utilize a single glycinergic narrow-field bistratified AC, called the All, to relay signals to both sublaminae and through cone DBCs and HBCs onto RGCs (Famigliette and Kolb, 1975; Strettoi et al., 1992; Tsukamoto et al., 2001; Völgyi et al., 2004). At light onset, rod DBCs provide a glutamatergic excitatory drive to the All ACs (Figure 1.2, 1.3A). All ACs make a sign-inverting glycinergic synapse with cone HBCs or OFF RGCs. They also make a sign-conserving gap junction (electrical synapse) with ON cone BCs (Famiglietti and Kolb, 1975; Strettoi et al., 1990). To broaden the retinal operating range to higher light levels (van Genderen et al., 2009; Völgyi et al., 2004)., rods use a secondary pathway to initiate visual signaling via gap junctions between rod spherules and cone pedicles or a tertiary pathway via a glutamateric synapse with OFF cone BCs (Figure 1.3B \& C; Raviola and Gilula, 1973; Soucy et al., 1998; Tsukamoto et al., 2001). 


\subsection{The Diversity of Amacrine Cells}

The retina encodes more than just light increments and decrements and it is hypothesized that the 30 morphologically different AC types and their synaptic organization plays this role. In fact, it is clear that one AC type, the starburst AC (SAC) is critical to the direction selectivity found in several RGC types (Yoshida et al., 2001; Werblin, 2011). With this and a few other examples, the remaining ACs are characterized primarily by their morphology, e.g., dendritic field size, shape and lamination pattern within the IPL (MacNeil and Masland, 1998; MacNeil et al., 1999) and the neurotransmitter/neuromodulators that they express (Karten and Brecha, 1983; Vaney, 1991; Zhu et al., 2014). Wide-field ACs (WF-ACs) have large dendritic arbors $(\geq 400 \mu \mathrm{m})$ and are primarily GABAergic (Pourcho and Goebel, 1983). Most have narrow lamination patterns and stratify within a single IPL laminae or within multiple On or Off sublaminae. They are likely to provide widespread lateral inhibition restricted to either the On or Off pathway (MacNeil and Masland, 1998; Pérez de Sevilla Müller et al., 2007; Pourcho and Goebel, 1983). Narrow-field ACs (NF-ACs) have small dendritic arbors $(<125 \mu \mathrm{m})$ and are primarily glycinergic. Most have processes that stratify within more than one IPL sublamina (Menger et al., 1998). They are likely to provide local inhibition between the On and Off pathways (MacNeil and Masland, 1998; MacNeil et al, 1999). Medium-field ACs have dendritic arbors that extend $\sim 200 \mu \mathrm{m}$, and include starburst and DAPI3 ACs, which are involved in directional selectivity, lateral inhibition and gain control, respectively (Werblin, 2011; Yoshida et al., 2001). 


\subsection{ON and OFF RGCs and Their Receptive Field Organization}

There are 15-20 different morphological/functional RGCs types in the mammalian retina. Each type is thought to represent a unit that specifically processes an aspect of the visual image and the fundamental building blocks of all subsequent visual processing. Like the ACs, RGCs are characterized morphologically on the basis of their soma diameter, dendritic field size and IPL stratification pattern (Rockhill et al., 2002; Sun et al., 2002).

\subsubsection{ON and OFF RGCs}

Most RGCs have receptive fields (RFs) with specific spatial organization; an excitatory center and a coextensive antagonistic surround (Rodieck and Stone, 1965). Functionally, RGCs have been grouped as ON or OFF, based on their excitatory response to light increments and decrements presented within their RF center. ON RGCs increase their firing rate in response to a luminance increment within their RF center; whereas OFF RGCs increase their firing rate in response to a luminance decrement in their RF center (Kuffler, 1953). ON RGCs have an OFF surround and OFF RGCs have an ON surround (Kuffler, 1953; Wiesel, 1959; Rodieck and Stone, 1965; Stone and Pinto, 1993). The RGC RF excitatory center represents the summation of excitatory signals from BCs as well as local lateral inhibition via ACs. The recruitment of BCs is limited spatially by the span of the RGC dendritic arbor (Werblin, 1991) and the RF center response represents a relatively linear summation that can be quantified using spots of increasing diameter (Kuffler, 1953; Rodieck and Stone, 1965; 
Sagdullaev and McCall, 2005). For example, small spots of increasing diameter and of preferred contrast (positive contrast for ON, and negative contrast for OFF RGCs) evoke increasing excitatory responses that reaches a maximum when the spot diameter matches the dendritic field size (Figure 1.4). As the spot diameter increases beyond the RF center, the excitatory response declines as a result of surround inhibition. This functional distinction was subsequently correlated with their dendritic stratification in the IPL On and Off sublaminae (Nelson et al., 1978; Figure 1.2).

\subsubsection{Transient and Sustained RGCs within the ON and OFF Classes}

Both ON and OFF RGCs can be divided into two subgroups based on the temporal properties of their excitatory response within RF center (Cleland et al, 1971, 1973; Kuffler, 1953). To a light stimulus of appropriate sign (light increment for ON and decrement for OFF), sustained RGCs respond with an initial high frequency component (peak) followed by a steady-state component (maintained) that is matched to the duration of the stimulus. Transient RGCs respond with high frequency firing rate at stimulus onset that quickly returns to the level of spontaneous activity, usually in less than one second when the stimulus is at

least $1.5 \mathrm{sec}$. The basis for RGC temporal specificity may originate from intrinsic properties of sustained and transient BCs that provide their excitatory drive (Awatramani and Slaughter, 2000), although inhibitory circuits clearly also modulate RGC temporal responses (Caldwell and Daw, 1978; Eggers and Lukasiewicz, 2011). 


\subsubsection{Inhibitory Surround of RGC Receptive Field}

The inhibitory surround is spatially concentric with the RF center, although it extends beyond it. The surround results from a combination of local and widespread lateral interactions among the BCs, ACs and RGCs. In the OPL, HCs exert inhibitory influences on PRs and BCs to generate BC surround antagonism (Sterling, 1995; Thibos and Werblin, 1978; Thoreson and Mangel, 2012). The exact synaptic mechanism of HC-mediated inhibition remains highly debated. The diverse mechanisms that are potentially responsible include: modulation of $\mathrm{PR} \mathrm{Ca}^{2+}$ channels via hemichannels (ephaptic mechanism) and/or changes in $\mathrm{pH}$ at these same synapses (chemical mechanism) to regulate PR glutamate release (Hirasawa and Kaneko, 2003; Kamermans and Spekreijse, 1999; Klaassen et al., 2011; Thoreson and Mangel, 2012; Vroman et al., 2013). A recent study shows that at cone synapses, HCs use both ephaptic and chemical mechanisms that play distinct temporal kinetics to adjust the output of cones, and GABA release at GABA autoreceptors on HCs to modulate the two mechanisms (Kemmler et al., 2014).

In the IPL, ACs release neurotransmitters to mediate complex operations including not only temporal and spatial tuning of RF center-surround (Cook and McReynolds, 1998; Murphy and Rieke, 2006; Nobles et al., 2012; Roska et al., 2006; van Wyk et al., 2009), but also contrast enhancement, motion detection and direction selectivity (Manookin et al., 2008; Münch et el., 2009; Russell and Werblin, 2010; Yoshida et al., 2001). 


\section{Inhibitory Receptors and Inhibitory Circuits in the Inner Retina}

In the retina, GABA and glycine are both used as inhibitory neurotransmitters by ACs and in approximately equal proportions. Ionotropic GABA receptors ( $\left(A B A_{A} R s\right.$ and $\left.G A B A_{C} R s\right)$ and glycine receptors (GlyRs) belong to the pentameric acetylcholine receptor (nAchR) superfamily. They are ligand-gated transmembrane chloride channels which increase a chloride conductance and hyperpolarize cells (Cascio, 2006; Figure 1.5).

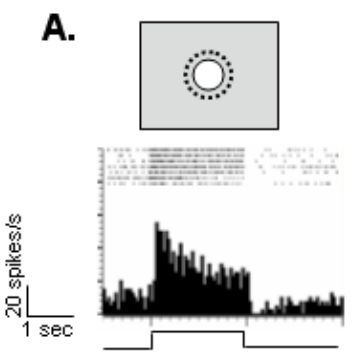

A.

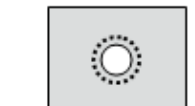

B.

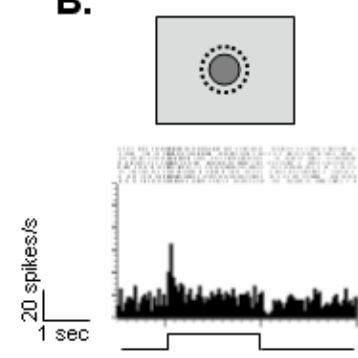

\section{ON-center RGCs}

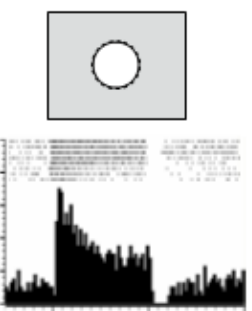

OFF-center RGCs
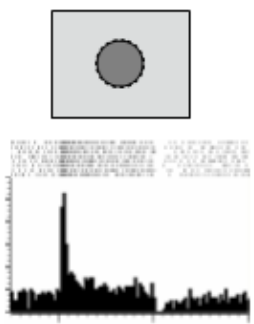
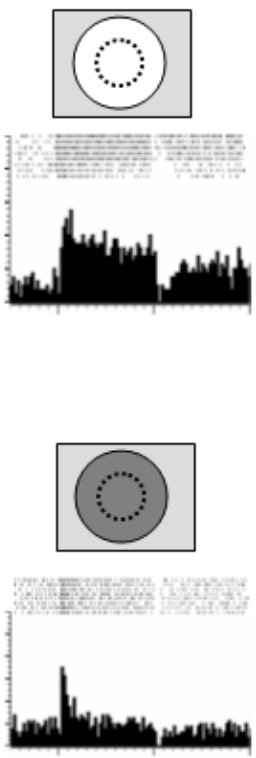

Figure 1.4. ON and OFF RGCs RF center summation. Representative lightevoked responses of wild type (WT) mouse ON- (A) and OFF- center (B) RGCs. Spot stimuli of varying diameter (light increment for ON and decrement for OFF RGCs are presented on the same adapting background) elicit excitatory response. Spots with RF center size evoke the optimal response. In the presence of larger spots, RF center and surround are stimulated simultaneously and the 
excitatory response is reduced. (modified from dissertation proposal by Nobles, 2009)

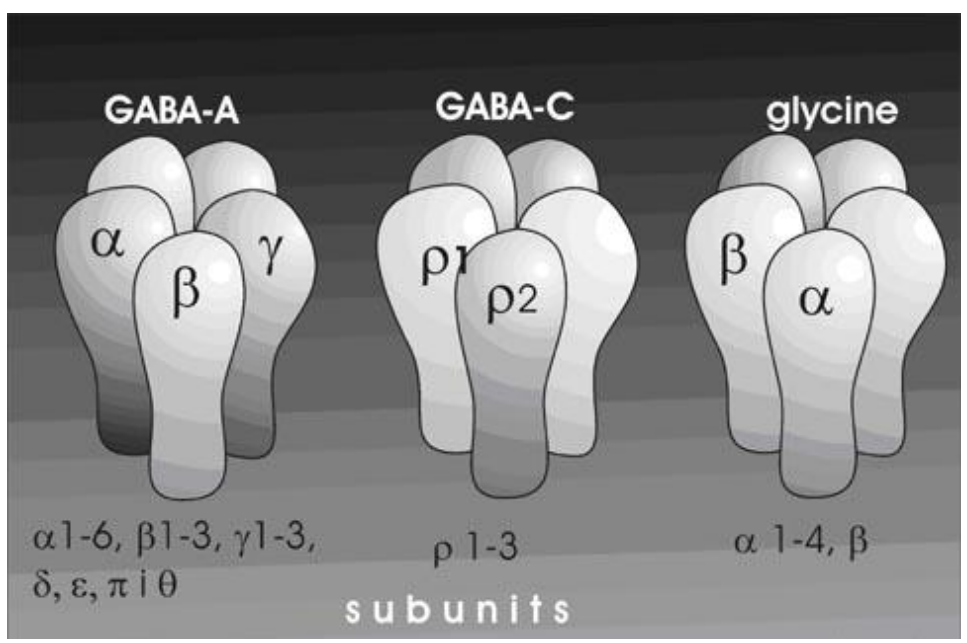

Figure 1.5. Pentameric structure of ionotropic inhibitory receptors and their subunits. The basic GABA $A_{A}$ receptor is composed of $\alpha, \beta$ and $y$ subunits. The $\rho 1$ subunit is critical for $\mathrm{GABA}_{C}$ receptor assembling while the composition of the receptor still needs to be determined. The synaptic glycine receptor is composed of $2 \alpha$ homomeric subunits and 3 homomeric $\beta$ subunits. (http://www.ifcc.org/ejifcc/vol15no3/ 150309200408. htm) 


\section{1 lonotropic GABA Receptors}

In mammalian retinas, the $\mathrm{GABA}_{A} R$ receptor is composed of $\alpha, \beta$ and $\gamma$ subunits (Figure 1.5), whereas the $G A B A_{C} R$ may be either homomeric for the $p 1$ subunit or heteromeric including both $\rho 1$ and $\rho 2$ subunits. If both are expressed, only the $\rho 1$ subunit is required for receptor assembly (Enz, 2001; McCall et al., 2002). The functional properties of $G A B A_{A} R s$ and $G A B A_{C} R s$ have been well studied, using their selective antagonists, e.g., picrotoxin, Bicuculline or SR95531, which block $\mathrm{GABA}_{\mathrm{A}} \mathrm{Rs}$ and 1,2,5,6-Tetrahydropyridin-4-yl) methylphosphinic acid (TPMPA) which blocks $\mathrm{GABA}_{C}$ Rs. $\mathrm{GABA}_{A}$ Rs and $G A B A_{C}$ Rs mediate spontaneous and light-evoked inhibitory postsynaptic currents (sIPSCs and elPSCs) with distinct kinetics. GABA ${ }_{A} R$ sIPSCs and elPSCs exhibit fast time to peak and fast decay; whereas GABA $R$ sIPSCs and elPSCs have slow time to peak and slow decay (Table 1.1; reviewed in Eggers and Lukasiewicz, 2011). Light-evoked GABAergic inputs have been recorded at the axon terminals across all types of $B C s$. These inputs are mediated by both $G A B A_{A} R s$ and $G A B A_{C} R s$ and their relative proportions are related to the $\mathrm{BC}$ type. Rod $\mathrm{BC}$ receive a large $\mathrm{GABA}_{C} \mathrm{R}$-mediated input and a smaller $\mathrm{GABA}_{\mathrm{A}} \mathrm{R}$-mediated input. Compared to rod $\mathrm{BCs}$, ON cone $\mathrm{BCs}$ have larger $\mathrm{GABA} \mathrm{A}_{\mathrm{A}} \mathrm{R}$-mediated input while $\mathrm{GABA} \mathrm{A}$ mediated input still dominates. In OFF cone $B C s, G A B A_{A} R s$ and $G A B A_{C} R s$ mediate the inputs in relatively equal amounts (Eggers et al., 2007; Eggers and Lukasiewicz, 2011). The differential distributions of $G A B A_{A} R-$ and $G A B A_{C} R-$ mediated currents on the $\mathrm{BC}$ axon terminals lead to distinct shaping of their excitatory output. Furthermore, $\mathrm{GABA}_{\mathrm{A}} \mathrm{Rs}$ are suggested to mediate inhibitory 
inputs onto ACs and RGCs to modulate spatial properties of RGC RF (Cook and McReynolds, 1998; Eggers and Lukasiewicz, 2010; Flores-Herr et al., 2001; O'Brien et al., 2003).

Table 1.1 Parameters for GABAergic sIPSCs and elPSCs from ON cone BCs (modified from Eggers et al., 2007)

\begin{tabular}{|c|c|c|c|}
\hline \multirow{2}{*}{ Receptor } & SIPSC & \multicolumn{2}{|c|}{ elPSC } \\
\cline { 2 - 4 } & $\mathrm{T}_{\text {decay time }}(\mathrm{ms})$ & Time to peak $(\mathrm{ms})$ & $\mathrm{T}_{\text {decay time }}(\mathrm{ms})$ \\
\hline GABA $_{\mathbf{A}}$ & $2.1 \pm 0.5$ & $136.3 \pm 5.8$ & $87.8 \pm 19.4$ \\
\hline GABA $_{\mathbf{C}}$ & $5.2 \pm 0.4$ & $207 \pm 21.3$ & $242.5 \pm 52.0$ \\
\hline
\end{tabular}

\subsection{Ionotropic Glycine Receptors}

While $\mathrm{GABA}_{\mathrm{A}} \mathrm{Rs}$ and $\mathrm{GABA} \mathrm{A}_{C} \mathrm{Rs}$ are the most well characterized inhibitory receptors in the retina; GlyRs are less well understood primarily because of the lack of selective antagonists to target specific subunits. Five different GlyR subunits have been identified with four $\alpha$ subunits ( $\alpha 1, \alpha 2, \alpha 3$, and $\alpha 4)$ and one $\beta$ subunit (Figure 1.5). Synaptic GlyRs also are pentameric receptors composed of three $\beta$ subunits and two $\alpha$ subunits of a single type (reviewed in Betz and Laube, 2006; Dutertre et al., 2012). GlyR $\alpha$ and $\beta$ subunits are required for assembly, ligand binding and gating of the receptor (Grudzinska et al., 2005; Kneussel and Betz, 2000). The $\beta$ subunit binds the structural protein, gephyrin, which is 
essential for postsynaptic clustering of GlyRs to the cell membrane (Vannier and Triller, 1997). All five GlyR subunits are expressed in mouse retina with most of the expression located in the IPL (Dutertre et al., 2012; Wässle et al., 2009). Consistent with their general role in all glycinergic receptors, GlyR $\beta$ subunits are expressed throughout the IPL. The four GlyRa subunits show distinct expression patterns across the IPL sublaminae (Figure 1.6). GlyRa1 is most prominent in the Off sublamina. GlyRa2 is ubiquitously distributed throughout the IPL. GlyRa3 is expressed in four distinct bands in the IPL with the highest expression within its Off sublamina. GlyRa4 is expressed throughout the IPL with highest expression in a small band at the border of ON sublamina $3 / 4$ and in close proximity to the choline acetyl transferase (ChAT) where ON starburst ACs processes ramify (Heinze et al., 2007; Figure 1.7).

Consistent with the differences in their expression patterns, the four different GlyR $\alpha$ subunits ( $\alpha 1-4)$ are expressed on different retinal cell classes. In addition, the subunit composition imposes unique kinetic properties on the sIPSCs (Table 1.2). Initial, characterizations of the kinetics of these subunits were conducted at $25^{\circ} \mathrm{C}$ and showed the fastest conducting GlyRs express the $\alpha 1$ subunit (decay time constant $\mathrm{\tau} \sim 4 \mathrm{~ms}$ ). GlyRs expressing the $\alpha 2$ and $\alpha 4$ subunits have significantly slower kinetics (т $\sim 27 \mathrm{~ms} ; ~ \sim 66 \mathrm{~ms}$, respectively). The a3 subunit mediates inputs with medium fast kinetics ( $~ \sim 11 \mathrm{~ms}$ ) (Majumdar et al., 2007; Wässle et al., 2009). 


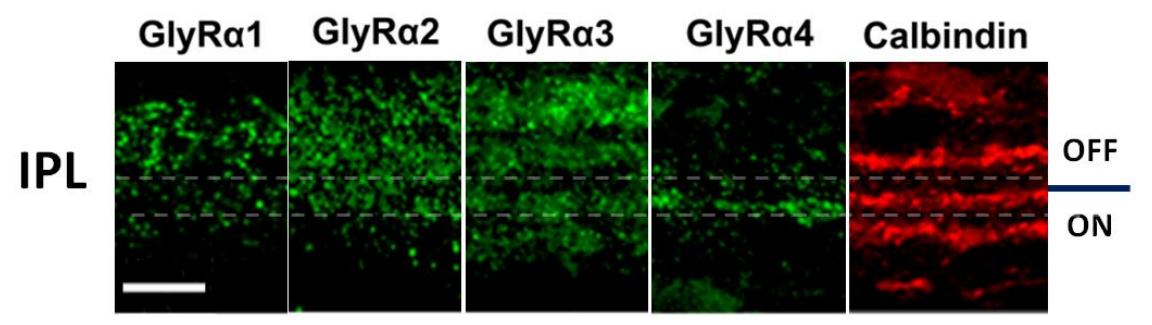

Figure 1.6. Localization of the four GlyRa subunits in the IPL. (A, D)

Normarski images of the five retinal layers. (B) GlyRa1 is predominantly

expressed in the Off sublamina of the IPL with sparse labeling in the OPL. (C)

GlyRa2 is evenly distributed throughout the IPL. (E) GlyRa3 is localized to four

distinct bands with the densest label in the Off sublamina. (F) GlyRa4 expression is highly concentrated between strata $3 / 4$ of the IPL. Scale bar $=50 \mu \mathrm{m}$.

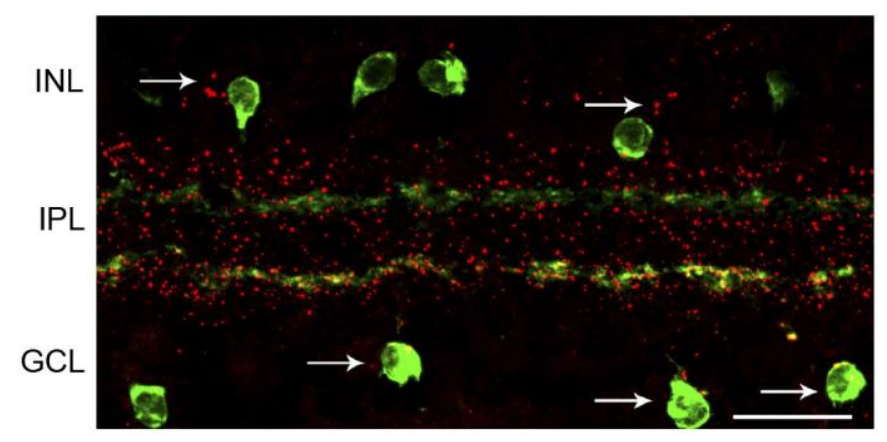

Figure 1.7. Vertical sections of a mouse retina double labeled for GlyRa4 (red) and ChAT (green). There is co-localization of GlyRa4 highly concentrated band and the ON-cholinergic stratum. Cholinergic cells in GCL are co-registered with GlyRa4-positive puncta (arrows), while cholinergic cells in INL are not (arrows). Scale bar= $20 \mu \mathrm{m}$. (Modified from Heinze et al., 2007) 
Table 1.2 Different cell types express specific GlyRa subunits with distinct kinetics.

\begin{tabular}{|l|c|l|l|}
\hline Subunit & Tdecay time(ms) & Kinetics & \multicolumn{1}{|c|}{ Cell Type } \\
\hline GlyRa1 & $3.9 \pm 2.5$ & Fast & OFF cone BCs; rod BCs; A-type RGCs \\
\hline GlyRa2 & $27 \pm 6.8$ & Slow & NF-ACs; WF GABAergic ACs \\
\hline GlyRa3 & $11.2 \pm 0.2$ & Mid-fast & All ACs \\
\hline GlyRa4 & $66.2 \pm 90$ & Very slow & ON starburst ACs \\
\hline
\end{tabular}

Electrophysiological studies in mouse retina have shown that the predominate inhibitory inputs onto OFF cone BC terminals are glycinergic and that they also participate in inhibition on rod BCs. No glycinergic inputs have been observed in ON cone BCs (Eggers et al., 2007; Ivanova et al., 2006). GlyRa1 is the only GlyRa subunit mediating glycinergic currents in OFF cone BCs (Ivanova et al., 2006). The distribution of subunit specificity among specific types of RGCs and ACs is less well described. GlyRa1 is the only GlyRa subunit mediating glycinergic currents in A-type ganglion cells in mouse (Majumdar et al., 2007). GlyRa2 has been reported to mediate glycinergic currents in type $5 / 6$ and 7 NF-ACs (Weiss et al., 2008) and in displaced GABAergic WF-ACs (Majumdar et al., 2009). GlyRa3 appears to be responsible for glycinergic currents in All ACs (Weiss et al., 2008). Although there is very little electrophysiological data on GlyRa4, Majumdar et al (2009) reported that glycine currents in ON starburst 
ACs had very slow kinetics ( 50 to $70 \mathrm{~ms}$ ), indicating a role for GlyRa4. With distinct kinetics and distribution within IPL, the four GlyRa subunits could play unique roles in the inhibitory network in the retina to modify different properties of RGC activities. However, the contribution and mechanisms of GlyR $\alpha$ subunitspecific mediated inhibition have not been fully studied due to the lack of selective antagonist.

\subsection{Different Inhibitory Circuits Mediated by GABA and Glycine Receptors}

Amacrine cells target different postsynaptic cell classes in the IPL (BCs, $A C s$ and RGCs) releasing either GABA or glycine to activate $G_{A B A} R, G A B A_{C} R$ and GlyR currents. This forms a highly complex inhibitory network, which fine tunes visual signaling that culminates in the spiking activity of RGCs. Four basic inhibitory mechanisms are found in these networks: feedback, feedforward, serial and crossover inhibition (Figure 1.8). The first two produce direct inhibition from the pre to the post cells, whereas the latter two produce disinhibition in their postsynaptic targets.

Reciprocal feedback inhibition (Figure 1.8A) occurs between a presynaptic excitatory cell that receives inhibitory input from its postsynaptic target. In the retina, there are two well-characterized reciprocal feedback inhibitory circuits. The A17 AC mediates GABAergic feedback inhibition onto its presynaptic rod DBC, which modulates the temporal properties of the rod BC output (Dong and Hare, 2003; Lukasiewicz and Werblin, 1994; Menger and Wässle, 2000; Zhang and McCall, 2012). Although the identity of the postsynaptic AC is unknown, there also is a GABAergic feedback inhibition onto the OFF BC. In both circuits, 
$\mathrm{GABA}_{C} \mathrm{Rs}$ are the primary postsynaptic target of the reciprocal inhibition (Eggers and Lukasiewicz, 2010, 2011; Shields et al, 2000).

Feedforward inhibition (Figure 1.8B) occurs between an inhibitory presynaptic cell whose output directly reduces excitation in its postsynaptic target. In the retina, there are many examples of this form of inhibition. GABAergic ACs use this mechanism to enhance the RF surround of RGCs, via GABA $A_{A}$ s (FloresHerr et al., 2001; O’Brien et al., 2003; Roska et al., 2000). Similarly, glycinergic inhibition from ACs to RGCs modulates excitation of their RF center (Menger et al., 1998; Nobles et al., 2012; O’Brien et al., 2003).

\section{A. Feedback inhibition}

\section{Serial inhibition}
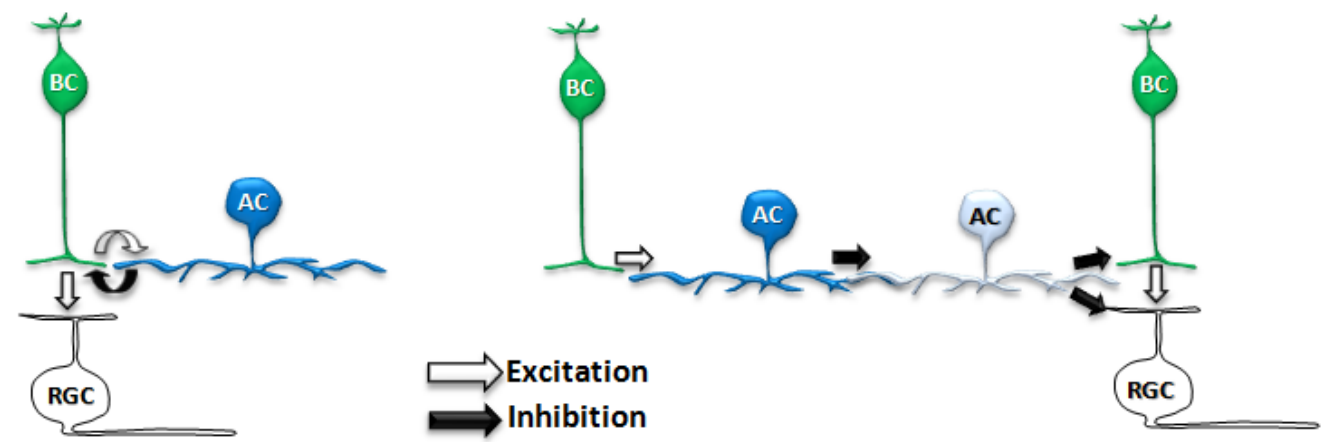

B. Feedforward inhibition

D. Crossover inhibition
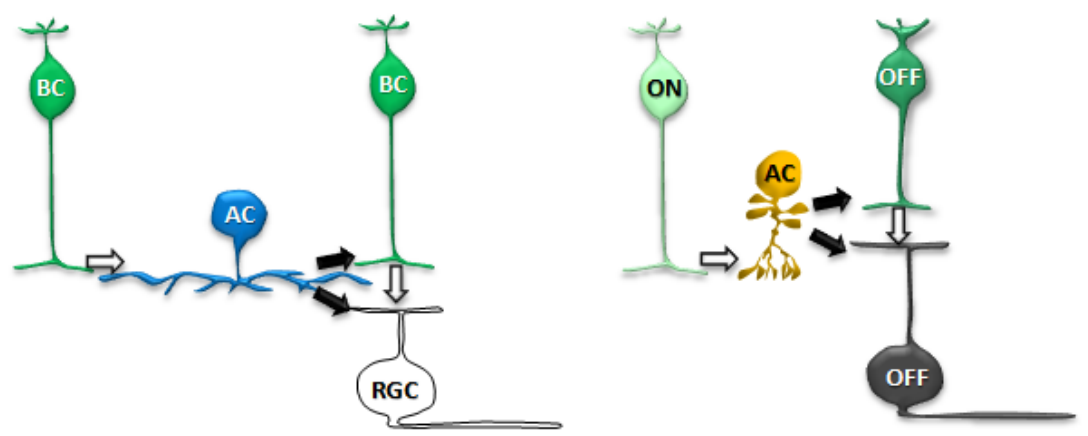

Figure 1.8. Illustrations of four basic types of inhibition. (A) Feedback inhibition modulates the release of glutamate from BCs to RGC. (B) Feedforward 
inhibition provides direct inhibition to BCs or RGCs. (C) Serial inhibition disinhibits the activities of BCs or RGCs. (D) Crossover inhibition enables reinforcement of signals by crosstalk between On and Off pathways.

Serial inhibition (Figure 1.8C), first observed in salamander retina (Zhang et al., 2007), has been observed in both BCs and RGCs in mammalian retina (Eggers et al., 2007; Eggers and Lukasiewicz, 2010; Russell and Werblin, 2010; Venkataramani and Taylor, 2010). Serial inhibition utilizes inhibition between ACs. The presynaptic $A C(A C 1)$ inhibitory output reduces excitation in its postsynaptic target (AC2), reducing its release of inhibitory neurotransmitter. The lowered inhibitory output of AC2 enhances excitation in its postsynaptic target producing what is called disinhibition. This mechanism has been observed in both BCs and RGCs. In $B C s$, serial inhibition spatially tunes the $B C$, using both $G_{A B} A_{A} R$ to $\mathrm{GABA}_{C} R$, and $G A B A_{A} R$ to GlyR inhibition (Eggers and Lukasiewicz, 2010, 2011). Direct evidence for serial glycinergic inhibition is not possible due to the lack of a selective GlyR antagonist. Although indirect, changes in signaling in the presence of the non-selective glycinergic antagonist, strychnine, are consistent with glycinergic control of GABAergic currents in RGCs (Caldwell and Daw, 1978; Stone and Pinto, 1992; Russell and Werblin, 2010). Finally, using subunit specific knockout mice, we have observed changes in inhibition that are consistent with 
subunit specific glycinergic control of GABAergic or other glycinergic subunit inhibition in BCs (Nobles et al., 2012).

Crossover inhibition mediates interactions between On and Off retinal parallel pathways (Figure 1.8D). Studies using electrophysiological strategies demonstrate that crossover inhibition can originate in either the On or the Off pathway and is usually glycinergic. Crossover inhibition is carried by NF-ACs via the bi- or multi-stratification of their processes in both On and Off IPL sublaminae and influences BCs, other ACs and RGCs (reviewed in Werblin, 2010). Because the excitatory input that initiates signaling occurs in the opposite pathway, stimulus onset removes inhibition and stimulus offset creates active inhibition in the postsynaptic cell. In this way, crossover inhibition enhances excitatory and inhibitory conductance in the postsynaptic cell, which creates more active antagonist surround within the IPL (Werblin, 2010). Furthermore, crossover inhibition is hypothesized to modify an inherently nonlinear synaptic current making it more linear to extend the operating range of RGC coding (Molnar et al., 2009). Many studies have shown modulation from On pathways to OFF BCs and RGCs and All cell is a candidate that mediates crossover interaction (Chen et al., 2010; Liang and Freed, 2010; Manookin et al., 2008; Murphy and Rieke, 2006, 2008; van Wyk et al., 2009). Although less studied, Off to On crossover pathway inhibition is also observed in the inner retina (Molnar et al., 2009).

New serial electron microscopy techniques describe other more complicated inhibitory synaptic organization (micronetworks) and indicate that nested feedback/feedforward and concatenated inhibition should be present 
within AC to AC synapses (Anderson et al., 2011; Marc and Liu, 2000). Whether or how these circuits translate into synaptic function and visual signaling remains to be determined. However, it is clear that there is diversity in AC inhibitory circuits that enables the retina to simultaneously process multiple aspects of visual signals in a highly complex manner.

\section{Specific Aims}

The preceding review demonstrates that we have made significant progress in understanding the retina's vertical circuits, but much less is known about the lateral inhibitory circuitry. The inner plexiform layer and the role of the diverse types of ACs is the new frontier in retinal research. Specifically, there remain many questions related to how the various types of lateral inhibition, as well as the various inhibitory receptor subunit composition contribute to processing the visual image to produce $20+$ representations at the level of RGC output. The focus of my dissertation was to begin to define the role of glycine receptor alpha subunit-specific inhibition in visual processing at RGC level using the mouse retina as a model. While the distinct distribution and decay kinetics of inhibitory postsynaptic currents of the four GlyR alpha subunits in the IPL suggest the possibility of subunit-specific inhibitory circuits in the IPL, we knew little about either subunit specific expression on RGCs or a subunit specific role in RGC visual processing. To begin to address these gaps in our understanding, I used subunit-specific knockout mouse lines and defined GlyRa2- and GlyRa3mediated inhibition in modulating RGC visual responses in circuits that formed 
their RF center (Nobles et al., 2012) and surround responses (Chapter III). I applied viral tool-mediated RNAi to define the role of GlyRa1-mediated inhibition in modulating visual activities of specific RGC, the OFFa ${ }_{\text {Transient }}$ cell (Zhang et al., 2014; Chapter IV). Finally, I surveyed the subunit-specific expression across 8 different RGC types (Chapter V). 


\section{CHAPTER II}

\section{EXPERIMENTAL MATERIALS AND METHODS}

\section{Animals}

Fifteen different mouse lines were used for the experiments (Table 2.1), including C57BL/6J wild type (WT) mice, GlyRa subunit KO mouse lines which lacked GlyRa2 (Glra2 ${ }^{--}$), or GlyRa3 (Glra3 $\left.{ }^{--}\right)$, or GlyRa4 (Glra4 ${ }^{-/}$), or both GlyRa2 and GlyRa3 (Glra2/3 $\left.{ }^{-/}\right), P V^{\text {cre }}$ mice which has YFP expressed in eight identified RGC types, TRHR mice which label a subset of DSGCs and ChATcre/Ai9 mouse line which labels SACs with tdTomato. The $P V^{C r e}$ or TRHR mice were crossed to GlyR a subunit single KO mouse lines to obtain several different mouse lines. All mice were maintained at the University of Louisville (all Chapters) or the Friedrich Miescher Institute (Chapter IV) on a 12:12h light/dark schedule. All experimental procedures were conducted in accordance with regulations described for the ethical care and treatment of animals in the Society for Neuroscience Policies on the Use of Animals in Neuroscience Research and with the approval of the individual Institutional Animal Care and Use Committees at the University of Louisville and the Friedrich Miescher Institute. 
Table 2.1 List of mouse lines used for experiments.

\begin{tabular}{|c|c|c|}
\hline Strain name & Source/Jackson lab stock\# & Chapter \\
\hline C57BL/6J & 000664 & III \\
\hline GIra2 $^{-/ 2}$ & Young-Pearse et al., 2006; 007065 & III \\
\hline GIra3 $^{-/-}$ & Harvey et al., 2004; 007065 & III \\
\hline GIra2/3/- & Glra2 $^{-/} \times$Glra3 $^{-/}$ & III \\
\hline GIra4 $^{-/-}$ & Created in our lab & V \\
\hline Pvalb & Hippenmeyer et al., 2005; 008069 & IV, V \\
\hline Thy $1^{\text {Stp-EYFP }}$ & Gift of J. Sanes; Buffelli et al., 2003; 005630 & IV, V \\
\hline$P V^{\text {cre }}$ & Pvalb ${ }^{\text {Cre }} \times$ Thy $1^{\text {Stp-EYFP }} ;$ Farrow et al., 2013; & IV, V \\
\hline $\mathrm{GIra2}^{-/} / \mathrm{PV}^{\mathrm{Cre}}$ & Glra2 $2^{-/} \times P^{\text {Cre }}$ & V \\
\hline Glra3 $^{-/} / P V^{\text {Cre }}$ & Glra3 ${ }^{-/} \times$PV Cre & V \\
\hline ChAT-cre/Aig & Gift of W. Guido; ChAT-cre (006410) X Aig(007905) & V \\
\hline TRHR-GFP & Gift of A. Huberman & V \\
\hline GIra2 ${ }^{-/ / T R H R}$ & Glra2 $^{-/} \times$TRHR-GFP & V \\
\hline GIra3'/TRHR & Glra3 $^{-/} \times$TRHR-GFP & V \\
\hline GIra4-/TRHR & Glra $4^{-/} \times$TRHR-GFP & V \\
\hline
\end{tabular}




\section{In Vivo Electrophysiology Recordings from Optic Nerve Fibers (Chapter III)}

\subsection{Surgical Preparation}

Surgical procedures were performed at light adapted levels and have been previously published (Nobles et al., 2012; Sagdullaev and McCall, 2005). Briefly, anesthesia was induced with an intraperitoneal injection of a Ringer's solution containing ketamine and xylazine. In WT mice the induction dose was $127: 12 \mathrm{mg} / \mathrm{kg}$. In G/ra2 ${ }^{-/}$and G/ra2/\% ${ }^{-/}$mice an increase of $12.5 \%$ was required to induce a surgical plane of anesthesia. Anesthesia was maintained throughout the experiments with supplemental subcutaneous injections $(50 \%$ of initial concentration) administered about every $45 \mathrm{~min}$. The head was secured in a stereotaxic frame (David Kopf Instruments) with ear cups and a bite bar. Body temperature was maintained at $37^{\circ} \mathrm{C}$ with a feedback controlled heating pad (TC1000; CWE). Topical Mydfrin (Phenylephrine hydrochloride ophthalmic solution 2.5\%) and Mydriacyl (Tropicamide ophthalmic solution 1\%; Alcon Labs, Inc.) were applied to dilate the pupils and paralyze accommodation. To prevent drying of the corneas, clear zero-powered lenses (Sagdullaev et al, 2004) moistened with artificial tears (Akwa Tears, Akorn, Inc.) were placed over the eyes. A craniotomy was performed anterior to the Bregma suture and the overlying cortex was removed using suction to expose the optic nerve. 


\subsection{Electrophysiology Recordings from Optic Nerve Fibers}

Action potentials were recorded extracellularly from single optic nerve axons using sharpened tungsten electrodes (A-M Systems, Inc.) with a final impedance between 70-100M $\Omega$. A reference electrode was inserted subcutaneously. Action potentials from single GC axons were isolated, amplified (X3+Cell, FHC), digitized at $15 \mathrm{kHz}$ (Power1401, CED) and stored for offline analysis. The isolated spikes were simultaneously displayed on an oscilloscope $(60 \mathrm{MHz}$, Tektronix Inc.) and computer monitor (Spike2, CED) and played over an audio monitor (AM7, Grass Instruments) to obtain direct feedback of the cell's response. Responses were analyzed offline using Spike2 software (Cambridge Electronic Design). Spikes were accumulated within a 50ms bin width and displayed as post-stimulus time histograms (PSTHs). Each average PSTH was smoothed by fitting it with a raised cosine function with a $50 \mathrm{~ms}$ smoothing interval to minimize alteration of the peak firing rate and maximize signal-to-noise ratio (Sagdullaev and McCall, 2005).

\subsection{Quantitative Characterization of RGC Visual Response Properties}

The position, spatial extent and RF center sign of each RGC were determined first using a handheld light source projected onto a screen located 25 $\mathrm{cm}$ from the anterior nodal point of the eye. All quantitative measures of RGC visual responses and receptive field properties were evoked by stimuli (VisionWorks; Vision Research) presented a CRT display monitor with a mean luminance of $20 \mathrm{~cd} / \mathrm{m}^{2}$ (Eizo E120 FlexScan FXC7). All stimulus and RF 
dimensions are presented in degrees of visual angle (corrected for monitor distance).

\subsection{Receptive Field Surround Analysis}

Annular stimuli were centered on the RF center and their inner diameter varied in eight steps $(0,270,372,544,754,1043,1587$ and $2616 \mu \mathrm{m}$ on the retina) while their outer diameter was fixed $(4425 \mu \mathrm{m})$. Each annulus inner diameter was presented eight times with a duration of 5 seconds and with 5 seconds inter-stimulus intervals. These trials were randomly interleaved with blank trials. For each inner diameter and for blank trials we computed an average PSTH (Figure 2.1). For ON RGCs the luminance of the annulus increased to $100 \mathrm{~cd} / \mathrm{m}^{2}$ above the mean luminance $\left(20 \mathrm{~cd} / \mathrm{m}^{2}\right)$ and for OFF RGCs the luminance decreased to $3 \mathrm{~cd} / \mathrm{m}^{2}$. This represents a $67 \%$ contrast change for both.

The mean spontaneous activity $(\bar{x} S A)$ was measured from blank trials where the monitor luminance was $20 \mathrm{~cd} / \mathrm{m}^{2}$ and a stimulus evoked response was considered significant if its amplitude was 3SEM above SA for excitatory or

3SEM below for suppressive responses. Figure 2.1 illustrates the quantitative measures that we derived from each average PSTHs. Each response was divided into a transient (within the first $0.4 \mathrm{sec}$ after stimulus onset; between cursors 1 and 2) and a maintained (the response that remained significantly different from the spontaneous activity from $0.4-5 \mathrm{sec}$; between cursors 2 and 3) component. The transient component is referred to as its peak firing rate when the response was excitatory or its minimum firing rate when the response was 
suppressive. The maintained component of the response is referred to as the mean firing rate (which can be either excitatory or suppressive). All responses were corrected for $\bar{X} S A$ to compute the response amplitude increments and decrements. For transient suppression, I excluded cells with a minimum firing rate equals to 0 spikes/s to avoid underestimating the magnitude. The inner diameter that evoked the maximal suppression is referred to as the optimal annulus. The duration of the response at the optimal inner diameter was determined as the time period when the response dropped below $\bar{x} S A$. The poststimulus peak amplitude at the optimal inner diameter was determined as the firing rate above $\bar{x} S A$ after stimulus offset
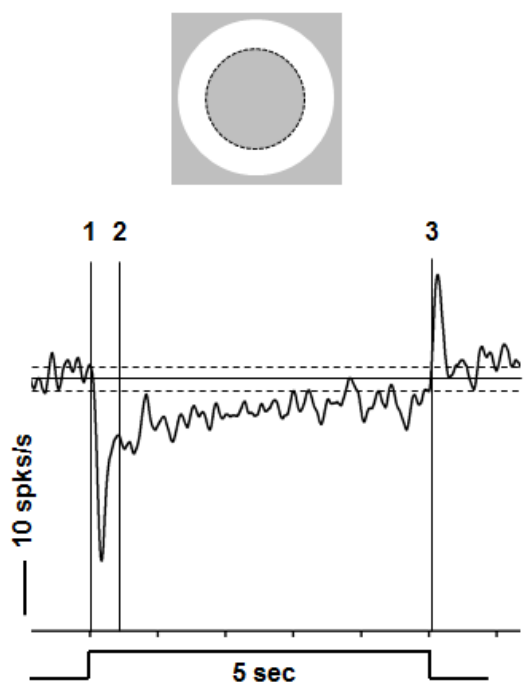

\section{Figure 2.1. PSTH response quantification during receptive field surround} stimulation. The spiking activity of an ON RGC is suppressed by a bright annulus (above) presented within its RF surround for a duration of 5 seconds. In the PSTH (below), the suppressive response begins when the firing rate crosses below -3SEM of $\bar{x} S A$ lower dotted line and ends when the response returns to 
the $\bar{x} S A$ (solid line). The vertical cursors define the transient response component (cursors 1-2, 0-0.4sec) and the maintained response component (cursors 2-3, 0.4-2.0sec).

\section{In Vitro Characterization of GlyR Subunit-specific Expression and Function (Chapter VI-V)}

\subsection{AAV Vector Construction}

The AAV vector plasmid AAV-Ef1a-NLStdTomato-H1 (Figure 2.2) was constructed by linearizing the viral vector AAV-Ef1a-NLStdTomato at its Pmll site and inserting an $\mathrm{H} 1$ promoter. A poly $(\mathrm{A})$ tail was added by seamless cloning using GeneArt ${ }^{\circledR} 2 x$ enzyme mix (Life technologies) and the vector was then linearized with Sall/Xbal. The synthesized shRNA cassettes (Sigma-Aldrich) were inserted after the $\mathrm{H} 1$ promoter (Figure 2.2). AAV was chosen because of its high efficiency and low immunogenicity (reviewed in McClements and MacLaren, 2013).

Two 21-mer and one 29-mer shRNA were designed and synthesized to target different regions of Glra1 mRNA. A scrambled shRNA, designed to no gene was used as a control. The efficiency of each of three Glra1 and a scrambled shRNA construct were assessed in cultured HEK293T cells after cotransfection with a plasmid expressing GlyRa1 (pCMV6-AC-GFP, carrying mouse Glra1 cDNA open reading frame (OriGene)). 


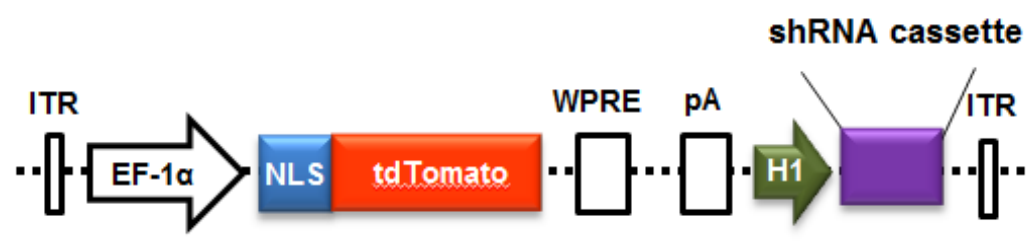

Figure 2.2. A schematic diagram of the AAV-RNAi vector backbone, pAAVEf1a-NLStdTomato- H1. The shRNA cassette (Scrambled or Glra1) was inserted after the $\mathrm{H} 1$ promoter. ITR, flanking inverted terminal repeats; EF-1 $\alpha$, elongation factor-1 alpha promoter; NLS, nuclear localization signal for the fluorescent protein tdTomato; WPRE, woodchuck hepatitis post-transcriptional regulatory element; $\mathrm{pA}$, poly $(\mathrm{A})$ tail.

\subsection{RNA Isolation and cDNA Preparation}

Forty eight hours after transfection of HEK293 cells, the mRNA level of Glra1 was measured. RNA was isolated with TRIzol ${ }^{\circledR}$ LS reagent (Invitrogen) according to a standard protocol including DNasel treatment (Promega) to remove residual genomic DNA. The cDNA was synthesized using $1 \mu \mathrm{g}$ RNA and random primers (Promega) according to SuperScript ${ }^{\mathrm{TM}}$ III Reverse Transcriptase kit (Invitrogen).

\subsection{RT-PCR}

RT-PCR was performed to determine mRNA levels of Glra1 using StepOne ${ }^{T M}$ Real-Time PCR System (Applied Biosystems). Each $20 \mu$ reaction 
mixture included: $2 \mu \mathrm{l}$ cDNA, $10 \mu$ SYBRGreen mix (Invitrogen) and $1 \mu \mathrm{l}$ Glra1 or 18s RNA primer set $(10 \mu \mathrm{M})$. For each cDNA sample, three PCR replicates were performed using each primer set. The PCR cycling conditions were: incubation at $50^{\circ} \mathrm{C}$ for $2 \mathrm{~min}$, denaturation at $95^{\circ} \mathrm{C}$ for $10 \mathrm{~min}$, and 40 cycles of $95^{\circ} \mathrm{C}$ for $15 \mathrm{sec}$ and $60^{\circ} \mathrm{C}$ for $1 \mathrm{~min}$. With $18 \mathrm{~s}$ RNA as internal control (Krol et al., 2010), the fold change of Glra1 in each cDNA sample was calculated using $\Delta \Delta \mathrm{C}_{\mathrm{T}}$ method.

Glra1 RT-PCR primer forward: 5'-CCGTCTGGCCTACAATGAAT-3'

Glra1 RT-PCR primer reverse: 5'-CACGTCTGTACATCCATCGG-3'

\subsection{AAV Production}

Recombinant AAVs (serotype 2/7) were made according to a standard triple-plasmid protocol, by co-transfection of HEK293T cells with the AAV vector plasmid, AAV helper plasmid (harboring Rep/Cap) and Ad-helper plasmid (pGHTI-adeno1). Transfected cells were lysed and treated with benzonase (Sigma-Aldrich Cat\# E8263). Packaged AAVs were concentrated and purified from total cell lysates by iodixanol gradient centrifugation (Sigma-Aldrich, Optiprep $^{\mathrm{TM}}$ ) and collected in the $40 \%$ iodixanol band. Genome copy (GC) number titration was evaluated using RT-PCR (Applied Biosystems, TaqMan ${ }^{\circledR}$ reagents). High titers were produced for both Scrambled shRNA: $1.36 \times 10^{12} \mathrm{GC} / \mathrm{ml}$ and Glra1 shRNA: $\left.1.14 \times 10^{12} \mathrm{GC} / \mathrm{ml}\right)$.

3.5 Viral Injections in the Dorsal Lateral Geniculate Nucleus (dLGN) 
Mice were sedated with Chlorprothixene $(5 \mathrm{mg} / \mathrm{kg})$. Anesthesia was induced and maintained throughout the procedure using Isoflurane administered through a mask mounted in front of the nose of the mouse. Body temperature was maintained at $37^{\circ} \mathrm{C}$ with a feedback-controlled heating pad. The head of mouse was secured in a stereotaxic frame with ear bars and a bite bar. The skull was exposed with a midline incision then leveled with reference to sagittal sutures. A craniotomy was performed between the Bregma and Lambdoid sutures to access to the left dLGN. Tips of borosilicate glass pipettes (inner diameter 50-100 $\mu \mathrm{m}$ ) were filled with 2-3 $\mu \mathrm{l}$ virus and positioned $2.25 \mu \mathrm{m}$ posterior and 2.25 $\mu \mathrm{m}$ left of Bregma and lowered 2.5-2.7 $\mu \mathrm{m}$ from the brain surface into the dLGN. Using pressure, 1-1.5 $\mu \mathrm{l}$ virus was injected into the dLGN. Four weeks post AAV injection RGC nuclei were positive for AAV induced tdTomato expression and expression was stable up to at least 10 weeks post injection. Animals, 4-10 weeks post injection of AAV-Glra1-shRNA and AAVScrambled-shRNA, were used for all experiments.

\subsection{Immunohistochemistry}

Using previously published techniques, retinas were dissected and fixed in 4\% paraformaldehyde in PBS for 15-20 min (Nobles et al., 2012). Whole mount retinas were incubated in blocking solution $(10 \%$ normal goat or donkey serum in PBX (0.5\% Triton-X 100 in PBS)) for 60 minutes, and then reacted with different combinations of antibodies in order to examine expression of GlyRa subunits on the dendrites of RGCs. The primary antibodies to label GlyRa subunits include a 
mouse monoclonal anti-GlyRa1 primary (1:500, Synaptic Systems Cat\# 146 111), goat polyclonal antibody to GlyRa2 (1:50, Santa Cruz Biotechnology Cat\# SC17279), rabbit polyclonal antibodies to GlyRa3 (1:100, Chemicon Cat\# AB5472) and GlyRa4 (1:100, Chemicon Cat\# AB9696). The secondary antibodies include donkey anti-mouse IgG Alexa 555 (1:200, Life Technologies Cat\# A31570), donkey anti-goat IgG Cy3 (1:200, Jackson ImmunoResearch Cat\# 115165003), goat anti-mouse IgG Cy3 (1:200, Jackson ImmunoResearch Cat\# 111165003), goat anti-rabbit Cy3 (1:200, Jackson mmunoResearch Cat\# 705165003). The IPL sublamination pattern was defined using goat anti-ChAT (1:200, Chemicon Cat\# AB144) primary and a donkey anti-goat lgG Alexa 405 (1:200, abcam Cat\# ab175664) secondary antibody. Alternatively, the boundaries of IPL were defined using Vectashield mounting medium with DAPI (Vector lab) or Hoescht (1:1000, Life technologies Cat\# H3570). To visualize their somatic and dendritic morphology, $P V^{C r e}$ RGCs filled with neurobiotin were reacted with Streptavidin-Alexa 633 (1: 200, Life Technologies Cat\# S21375) or StreptavidinCy2 (1:300, Jackson ImmunoResearch Cat\# 016220084).

\subsection{Confocal Image Acquisition and Co-localization Analysis}

Retinas were imaged both at the FMI (Chapter IV) and at the University of Louisville (Chapter V). At the FMI, images were acquired on a Zeiss LSM 700 confocal microscope. Z-stack images of neurobiotin-filled RGCs were acquired with $0.3 \mu \mathrm{m} z$-step using a $40 \times$ oil immersion lens, NA 1.2 ; or $0.17 \mu \mathrm{m}$ z-step using a $63 \times$ oil-immersion lens, NA 1.3. In Louisville, images were acquired on a 
Olympus FV1000 confocal microscope. Z-stack images of neurobiotin-filled RGCs were acquired with $0.6 \mu \mathrm{m}$ z-step using a $40 \times$ water immersion lens, NA 1.15; or $0.2 \mu \mathrm{m}$ z-step using a $60 \times$ oil-immersion lens, NA 1.4. For each RGC, 1 2 non-overlapping dendritic areas $(70 \times 70 \mu \mathrm{m})$ were randomly selected and imaged.

Prior to co-localization analyses, images were deconvolved using Huygens ${ }^{\circledR}$ software (Scientific volume imaging). For each RGC, we examined all the dendritic processes within the selected areas. Imaris (Bitplane) was used for analysis of GlyRa subunit expression. To evaluate co-localization a channel was built for each dendritic area (ImarisColoc tool), which contained only areas where GlyRa1 puncta were coincident with identified RGC dendrites. Thresholds were set to include most potential coincident pixels and exclude non-specific colocalization due to background noise. The total number of coincident GlyRa puncta ( $\left.P_{\text {Original }}\right)$ was counted (Imaris Spot function) and the total length of dendrite $\left(L_{\text {Dendrite}}\right)$ was measured (Imaris Filament function). To evaluate specific coincidence, I compared this coincidence rate to a coincidence rate $\left(P_{\text {Random }}\right)$ I measured when we when I flipped the GlyRa positive puncta channel 180 degree along its horizontal/vertical/diagonal axis. The corrected rate was computed as follows: Coincidence rate $=\left(P_{\text {Original }}-P_{\text {Random }}\right) / L_{\text {Dendrite }}$

\subsection{Whole Mount Retinal Preparation for Electrophysiological Recordings}

The mouse was sacrificed with $\mathrm{CO}_{2}$ followed by cervical dislocation. Eyes were removed and retinas were prepared and dissected under infrared 
illumination in Ringer's solution (in $\mathrm{mM}, 110 \mathrm{NaCl}, 2.5 \mathrm{KCl}, 1 \mathrm{CaCl}_{2}, 1.6 \mathrm{MgCl}_{2}$, $10 \mathrm{D}$-glucose and $22 \mathrm{NaHCO}_{3}$, bubbled with $5 \% \mathrm{CO}_{2} / 95 \% \mathrm{O}_{2}$, $\left.\mathrm{pH} 7.4\right)$. They were mounted in the center of a piece of filter paper (Millipore) with a $4 \times 4 \mathrm{~mm}$ square aperture and ganglion cell-side up in the microscope recording chamber. Alternatively, the retina was cut into four quarters and each quarter was mounted on cover slip separately for recording. During the experiment, the retinas were superfused with oxygenated Ringer's solution at $34-35^{\circ} \mathrm{C}$.

\subsection{Targeting RGCs Using Two-photon Microscopy}

In WT $P V^{\text {cre }}$ retina, RGCs with YFP-positive soma were targeted based on the soma size and the morphology of their dendrites (Farrow et al., 2013). AAV infected PV5 RGCs were targeted by their YFP-positive soma with tdTomatopositive nuclei.

\subsection{Electrophysiology and Pharmacology}

Electrophysiological recordings of both spontaneous currents and spiking as well as light-evoked currents and spiking were performed both at the FMI (Chapter IV) and at the University of Louisville (Chapter IV \& V). In both labs, whole cell recordings used an Axon Multiclamp 700B amplifier (Molecular Devices) and signals were digitized at $10 \mathrm{kHz}$ (National Instruments) and acquired using software written in LabVIEW (National Instruments). In Louisville, the acquisition rate for spiking activity and for spontaneous and evoked currents in WT $P V^{\text {cre }}$ RGCs was $10 \mathrm{kHz}$. Similarly spiking activity for WT PV5 (PV5WT), and RGCs infected with AAV-scrambled shRNA (PV5sc) or with AAV-Glra1- 
shRNA (PV5 $5_{\text {Glra1-KD }}$ ) were recorded at $10 \mathrm{kHz}$ at the FMI. However, the acquisition rate for RGC spontaneous and evoked currents was $1 \mathrm{kHz}$. PV5 RGC spiking activity was recorded in loose patch configuration using electrodes pulled from borosilicate glass electrodes (Sutter Instrument) with 3-5 $\mathrm{M} \Omega$ resistance and filled with Ringer's solution. RGC currents were recorded in under whole-cell voltage clamp mode, with 5-8 $\mathrm{M} \Omega$ electrodes filled with (in $\mathrm{mM}$ ) 112.5 $\mathrm{CsCH}_{3} \mathrm{SO}_{3}, 1 \mathrm{MgSO}_{4}, 7.8 \times 10^{-3} \mathrm{CaCl}_{2}, 0.5$ BAPTA, 10 HEPES, 4 ATP-Na $2,0.5$ GTP-Na3, 5 lidocaine $N$-ethyl bromide (QX314-Br), 7.5 neurobiotin chloride $(\mathrm{pH}$ 7.2 adjusted with $\mathrm{CsOH}$ ). All voltages were corrected for the measured liquid junction potential of $17 \mathrm{mV}$. The calculated $\mathrm{Cl}$ reversal potential is $-73 \mathrm{mV}$. EPSCs and IPSCs were isolated while holding the RGC membrane potential at $77 \mathrm{mV}$ and $0 \mathrm{mV}$, respectively. For pharmacological characterization of inhibitory inputs in RGCs, I blocked GABA $A_{A}$ ergic inputs by application of $15-20 \mu \mathrm{M}$ picrotoxin (Sigma-Aldrich), GABAcergic inputs by $50 \mu \mathrm{M}$ TPMPA and glycinergic inputs by $10 \mu \mathrm{M}$ strychnine.

\subsection{In Vitro Visual Stimulation}

Stimuli were generated using a DLP projector controlled by custom software. The light spectrum ranged from about 400 to $760 \mathrm{~nm}$ with a peak at $\sim 550 \mathrm{~nm}$. The sIPSCs of PV5 RGCs were recorded on a photopic background luminance of $24,000 R^{\star} \cdot \operatorname{rod}^{-1} \cdot s^{-1}$, while the ones of other PV RGCs were recorded under no illumination $\left(0 \mathrm{R}^{*} \cdot \operatorname{rod}^{-1} \cdot \mathrm{s}^{-1}\right)$. Stimulus-evoked responses of PV5 RGCs were recorded to a luminance decrement, a dark spot $\left(6,000 \mathrm{R}^{*}\right.$.rod 
${ }^{1} \cdot \mathrm{s}^{-1}$ ) on the same background or to a luminance increment, the offset of the dark spot back to background. The visual stimulation protocol began with 15 seconds at background luminance, followed by presentation of a stationary dark spot centered on the PV5 RGC soma. The duration of the stimulus was 2 seconds followed by a 5 second inter-stimulus interval and the outer diameter increased in six steps from $125 \mu \mathrm{m}$ to $1250 \mu \mathrm{m}$. Spiking responses were recorded first in loose patch mode to 5-8 repetitions of this stimulus protocol (25 PV5 RGCs) and eEPSCs and eIPSCs were recorded in whole cell voltage clamp mode to 3-5 repetitions of the protocol (15 PV5 RGCs). Eight PV5 RGCs were recorded using loose patch followed by whole cell recording.

\subsection{In Vitro Electrophysiological Data Analysis}

\subsubsection{Extracellular Recordings}

Spiking activity was analyzed using Spike2 as described previously (see chapter II, section 2.2). Spikes were accumulated within a $50 \mathrm{~ms}$ bin width and displayed as both raster plots of individual trials and PSTHs. Mean spontaneous activity was measured from the $15 \mathrm{~s}$ background illumination at the beginning of the stimulation protocol and this mean was subtracted from all measures of the stimulus evoked responses. Several aspects of the spiking response were measured from the stimulus-evoked average PSTH of the PV5 RGCs. To a luminance decrement, the transient peak amplitude ( $0-0.4 \mathrm{~s}$ of stimulus onset) and the sustained mean firing rate $(0.4-2 \mathrm{~s}$ of stimulus onset) were measured. To a luminance increment, the rebound peak firing rate was measured. 


\subsubsection{Whole Cell Patch Clamp Recordings}

Only RGCs with series resistance smaller than $25 \mathrm{M} \Omega$ were used for analysis. In Chapter V, 50\% series resistance compensation was applied to all the cells. sIPSCs were analyzed using Mini Analysis Program (Synaptosoft). Only sIPSCs that were well separated events with fast rise time, a single peak and an amplitude that exceeded 2 times of root mean square of the noise were used for analysis. The $10-90 \%$ rise time, frequency and interevent interval of these sIPSCs were measured and their decay kinetics were estimated from the time at which the sIPSC declined to $37 \%$ of its peak amplitude $\left(D_{37}\right.$; Chapter IV) or a least-square fit of $10-90 \%$ of the decay phase, using a single exponential decay function ( $\mathrm{T}$; Chapter V). The decay time $\left(\mathrm{D}_{37}\right)$ for PV5WT sIPSCs acquired using 10 and $1 \mathrm{kHz}$ ( $\mathrm{n}=5$ and 7 cells) did not differ $(2.74 \pm 0.04 \mathrm{~ms}$ vs. $2.6 \pm 0.1$ $\mathrm{ms}, p>0.05)$ and were combined for analysis. For comparisons to $\mathrm{PV} 5 \mathrm{sc}$ and

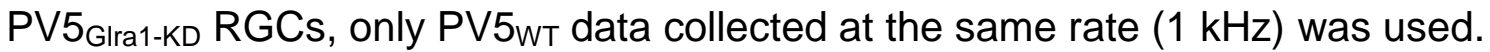

Stimulus-evoked eEPSCs and elPSCs of PV5 RGCs were analyzed using Clampfit (Axon Instruments). For each eEPSC and eIPSC, the transient peak (0$0.4 \mathrm{~s})$ and mean sustained current (0.4-2s) at stimulus onset and the charge transfer at stimulus offset were measured and corrected for average baseline, measured from 2 s before each stimulus onset.

\section{Statistical Analysis}

Statistical analyses were performed using Prism5 Software (GraphPad). Distributions of each parameter were tested for normality using the D'Agostino 
and Pearson omnibus test and appropriate parametric or non-parametric statistical analyses were used. For each morphological and electrophysiological measure, t-test with Welch's correction was used to compare RGCs with different genotypes or antagonist application, or infected with different AAVs. Student's paired $t$ tests were used to compare $\mathrm{P}_{\text {Original }}$ and $\mathrm{P}_{\text {Random }}$ of the same dendritic area, and responses of the same RGC in control and in the presence of antagonist. Two-way ANOVA with Bonferroni's post-hoc tests was used to compare response components across spot size across RGCs with different genotypes or antagonist application, or infected with different AAVs. The Kolmogorov-Smirnov test was used to determine whether the distributions of the decay time constants between two datasets were significantly different. In all figures, data are plotted as the mean \pm SEM and significance is indicated as ${ }^{*} p<0.05,{ }^{* *} p<0.01,{ }^{* * *} p<0.001$. 
CHAPTER III

\title{
THE ROLE OF GLYRa2- AND GLYRa3-MEDIATED INHIBITION IN THE RECEPTIVE FIELD SURROUND IN RETINAL GANGLION CELLS
}

\section{Introduction}

\begin{abstract}
At the first synapse in the retina between the photoreceptors and the bipolar cells, the On and Off pathways segregate and create the ability of the retina to respond to light increments and decrements. The culmination of these On and Off parallel information streams create RGCs with receptive fields that respond to light onset (ON), light offset (OFF) and both onset and offset (ON/OFF). As early as the bipolar cell, a receptive field exists with an excitatory RF center, and a coextensive inhibitory surround (Werblin and Dowling, 1969; Werblin, 1991). The same RF organization is found at the level of the retinal ganglion cell. Almost all RGCs have a RF center (Kuffler, 1953; Lukasiewicz and Werblin, 1990; Rodieck and Stone, 1965; Werblin, 1991), and a coextensive antagonistic surround (Kuffler, 1953; Rodieck and Stone, 1965; van Wyk et al., 2009). ON-center RGCs have OFF surrounds, whereas OFF-center RGCs have ON surrounds. This means that a stimulus of the same contrast that evokes an excitatory response within RF center at stimulus onset (light increment or light
\end{abstract}


decrement) produces a suppressive response within the isolated RF surround (Kuffler, 1953; Rodieck and Stone, 1965).

The RF surrounds of BCs arise from feedback inhibition via horizontal cell inputs to the photoreceptors, although the exact mechanism is not known (Sterling, 1995; Thibos and Werblin, 1978; Thoreson and Mangel, 2012; Werblin, 1974). The RF surrounds of RGCs are conveyed from the BC input but are augmented by AC-mediated inhibitory circuits (Eggers et al., 2007; Eggers and Lukasiewicz, 2010; Farrow et al., 2013; Flores-Herr et al., 2001; Protti et al., 2014). The AC-mediated circuits that contribute to the RF surround in the inner retina are poorly understood and we do not know if they vary between the On and Off pathways. As an added complication, within the On and Off pathways another pair of parallel pathways appears to be related to the temporal nature of the $R G C$ response and whether it is tuned to transient or sustained stimuli, which also are likely to be related to BC and AC-mediated circuits (Caldwell and Daw, 1978; Eggers and Lukasiewicz, 2011; Nobles et al., 2012).

Glycine and GABA are the primary inhibitory neurotransmitters utilized by ACs and help to divide the morphologically diverse ACs into two general classes: narrow field glycinergic ACs whose processes ramify across the On and Off strata of the inner plexiform layer (McNeil and Masland, 1998; Menger et al, 1998) and wide field GABAergic ACs whose processes ramify within the IPL On or Off sublaminae. As a consequence of the laminar location of their processes, narrow field ACs are thought to mediate local inhibition across the On/Off pathways (O'Brien et al, 2003; Chen et al, 2010; Nobles et al, 2012), whereas 
wide field GABAergic ACs mediate more widespread inhibition primarily confined within a lamina (Cook and McReynolds, 1998; Flores-Herr et al., 2001; Roska et al., 2000). This idea of local verses widespread inhibition has been translated into an RF surround that is formed by both local glycinergic inhibition, most likely coextensive with the RF center, and lateral GABAergic inhibition, most likely contributing primarily to the RF surround outside the RF center.

Several observations suggest, however, that glycinergic inhibition also might modulate more widespread GABAergic inhibition in RGCs using serial inhibitory circuits. For example, some WF-ACs express glycine receptors (Majumdar et al, 2009) and both NF and WF ACs participate in morphologically identified concatenated AC circuits (Anderson et al., 2011; Chen et al., 2011; Weiss et al, 2009). Glycinergic control of GABAergic currents in RGCs has been observed indirectly, using the glycinergic antagonist, strychnine. In the presence of strychnine, the light evoked RGC RF center excitatory and surround inhibitory response is shorten or abolished, indicating increased GABAergic inhibition within the RF center (Caldwell and Daw, 1978; Stone and Pinto, 1992). Glycinergic regulation of GABAergic feedback inhibition also has been observed in the RF center of a RGC type referred to as the local edge detector (Russell and Werblin, 2010). Moreover, we have observed changes in light evoked RGC responses in the absence of GlyR $\alpha$ subunits that only can be explained by glycinergic control of GABAergic or glycinergic inhibition at the level of the BC (Nobles et al., 2012). 
Both previous publications (Haverkamp et al, 2003; Young-Pearse et al., 2006) and our own work indicate that the retinas of GlyRa2 (Glra2 $\left.{ }^{-/}\right)$or GlyRa3 $\left(\right.$ Glra3 $\left.^{-/}\right)$subunit specific knockout mice develop without gross morphological abnormalities. ERG assays indicate that outer retinal function is normal in Glra2 $2^{-/-}$ mice (Young-Pearse et al., 2006) and that ACs normally express GlyRa2 or GlyRa3 retain excitatory synaptic inputs (Majumdar et al., 2009; Weiss et al., 2008). Expression of the calcium binding protein, calbindin, continues to show three characteristic WT AC bands in the IPL in Glra2 ${ }^{-/}$and $\mathrm{Glra3}^{-/}$and Glra2/3/retinas. We have confirmed that the expression of GlyR a subunits in the WT retina and their presence/absence in $\mathrm{KO}$ mouse retina using immunohistochemistry (reprinted from Nobles et al, 2012; Figure 3.1). Each single KO retina lacks expression of its targeted GlyRa subunit and the double KO retina lacks expression of both GlyRa2 and GlyRa3. In both single and the double KOs the expression pattern of the GlyRa1 and GlyRa4 remain the same as WT, consistent with an earlier publication (Heinze et al., 2007). Using RT-PCR we demonstrated that there is no upregulation at the transcriptional level of GlyRa2 in the absence of GlyRa3 (Nobles et al. 2012). All of the evidence is consistent and suggests that the absence of GlyRa2 and or GlyRa3 expression does not affect the gross retinal morphology or the expression of other subunits.

The pharmacological manipulation of glycine receptor mediated inputs is limited by available antagonists. Strychnine non-selectively blocks all alpha receptor mediated subunit inhibition. Therefore, GlyRa KO mice were used to more directly address the question of GlyR subunit specific contribution to the RF 
responses of ON- and OFF-center RGCs. The use of these mice can be thought of as a substitute for a subunit selective glycine antagonist. Our previous in vivo extracellular recordings from optic nerve axons of WT GIra2 ${ }^{-/}$, Glra3 ${ }^{-/}$and Glra2/3/- mice (Nobles et al., 2012) showed that both GlyRa2 and GlyRa3 containing receptors modulate the temporal response properties of RGC RF center, consistent with previous observations using strychnine (Caldwell and Daw, 1978; Stone and Pinto, 1992). GlyRa2 and GlyRa3 differentially contribute to visually-evoked RF center responses of RGCs that received RF center input from On and Off retinal pathways. In the On pathway, GlyRa2 and GlyRa3 work together to enhance the RF center excitatory responses at luminance increment and keep these responses more sustained, whereas only GlyRa2 contributes to suppressive response at luminance decrements. In the Off pathway, only GlyRa2 enhances the RF center responses. GlyRa2-mediated inhibition enhances the excitatory responses at luminance decrements and suppressive response at luminance increments. GlyRa3-mediated inhibition does not appear to contribute to Off pathway signaling in our RGC sample.

To study GlyR subunit specific contribution to the RF surround of ON- and OFF-center RGCs, I also used Glra2 ${ }^{-/}$, Glra3 ${ }^{-/}$and Glra2/3/- mouse lines. Using annular stimuli, I isolated the contribution of the RF surround and characterized the responses of ON and OFF WT RGCs that also were separated into sustained and transient subgroups. Similarly, I characterized these responses in G/ra2 ${ }^{-/}$, and GIra3 ${ }^{-/}$RGCs and compared them to WT. In addition, I compared the G/ra2 ${ }^{-/}$ or Glra3 ${ }^{-/}$RGCs to Glra2/3/ RGCs. My results define the contributions of 
GlyRa2 and GlyRa3 subunits to the suppressive RF surround of ON and OFF RGCs. My results strongly support the following conclusions: (1) neither GlyRa2 or GlyRa3 subunit-mediated inhibition modulates the RF surround of ON RGCs;

(2) GlyRa3 does not modulate the RF surround of OFF RGCs; and (3) only GlyRa2 modulates RF surround antagonism of OFF RGCs, where it enhances the suppressive surround at luminance decrements.

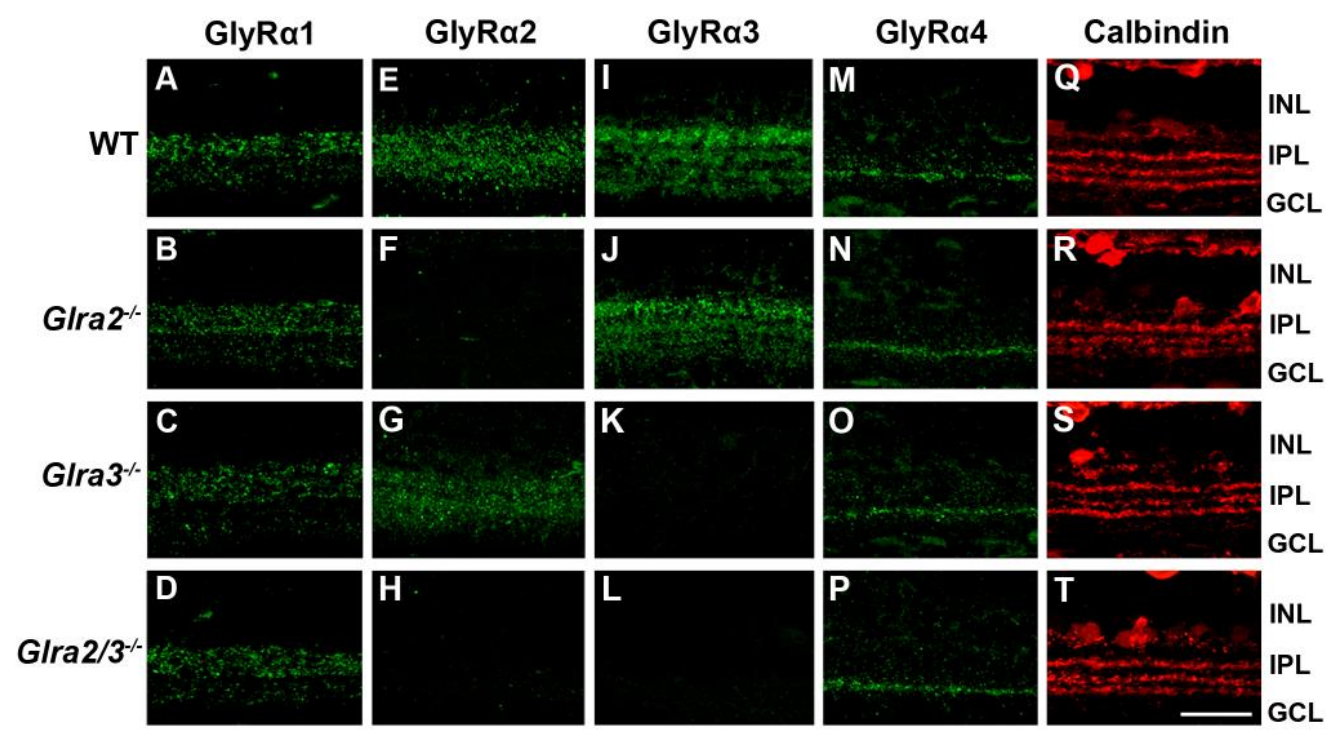

Figure 3.1. Vertical cross sections of mouse retina labeled for the four GlyR a subunits. (A-D) GlyRa1puncta are prominent in the outer strata of the inner plexiform layers across genotype. (E,G) GlyRa2 puncta are diffuse across all strata of the inner plexiform layer. $(\mathbf{F}, \mathbf{H})$ GlyRa3 puncta are absent in the Glra2 ${ }^{-1}$ and Glra2/3/-retina (I, J) GlyRa3 puncta are more prominent in the outer strata but are also evident to a lesser extent in the inner strata of the inner plexiform layer. (K, L) GlyRa3 puncta are absent in the G/ra3 ${ }^{-/}$and Glra2/3/- retina. (M-P) GlyRa4 puncta are localized to a distinct band within the inner plexiform layer and are evident across genotype. (Q-T) Retina from each genotype was labeled 
for calbindin (red) to illustrate normal IPL sublamination patterns for each genotype. Scale bar=20 $\mu \mathrm{m}$ (from Nobles et al., 2012).

\section{Results}

\section{Isolated RF Suppression is Found in Most WT ON and OFF RGCs}

To isolate and study RGC surround antagonism, I used annular stimuli with a fixed outer diameter of $4425 \mu \mathrm{m}$ on the retina and increasing inner diameter (ID; 0-2616 $\mu \mathrm{m}$, see also Chapter II, section 2.4). Annular stimuli with small IDs evoked excitatory responses from both WT ON and OFF RGCs, indicating that they result primarily from inputs to the RF center (Figure 3.2Ai). As annular inner diameter increased, the WT RGC excitatory response declined and passed through a null point (Figure 3.2Aii), where the annulus evoked little or no response. The null point occurs when the RF center excitatory input and the RF surround inhibitory input reach a balance. Further increases in annular inner diameter evoked a suppressive response, a significant reduction in the spontaneous activity level. This response indicates that the RF surround input

dominates and, in most cases, is the sole input to the RGC (Figure 3.2Aiii). There was usually an optimal annulus ID that evoked maximum suppression (Figure 3.2Aiii) and with further increase in the annulus ID, the depth of the response suppression declines because the area of surround stimulation decreases (Figure 3.2Aiv \& Av). The presence of a surround was observed in almost all WT ON and OFF RGCs (Table 3.1). In $9 \%$ of WT ON and $5 \%$ of WT OFF RGCs a surround was not recorded. In these RGCs, the RF center diameter was larger 
than the widest ID produced on my computer display. Thus, it is most likely that these RGCs had surround suppression but it could not be observed due to constraints of the stimulator.

In all WT RGCs, the suppressive surround response peaked $<400 \mathrm{msec}$ after stimulus onset. This transient response was followed by a maintained suppressive component whose duration varied compared to the $5 \mathrm{sec}$ stimulus duration. I defined the RGC RF surround optimal ID as the annulus that evoked the largest transient suppression (minimum transient firing rate). Although with the limited size of the display, the majority of WT RGCs, regardless of RF center sign, were examined with at least one ID greater than the optimal. By comparison, 45\% ON and $80 \%$ OFF WT RGCs were examined with two IDs greater than the optimal. In the remaining analyses I investigated the surround suppression at the optimal ID in all the RGCs that had suppression response, and the surround suppression as a function of the percent annular size compared to the optimal in RGCs examined with at least two annulus IDs greater than the optimal (Figure $3.2 \mathrm{~B})$.

Table 3.1 A comparison of RGC with surround suppression across genotype.

\begin{tabular}{c|cc|cc}
\hline & \multicolumn{2}{|c|}{ ON GC } & \multicolumn{2}{c}{ OFF GC } \\
\cline { 2 - 5 } & \# (\%) Suppression & Total & \# (\%) Suppression & Total \\
\hline WT & $51(91 \%)$ & 56 & $35(95 \%)$ & 37 \\
\hline Glra2 $^{-/}$ & $55(96 \%)$ & 57 & $51(93 \%)$ & 55 \\
\hline Glra3 $^{--}$ & $62(89 \%)$ & 70 & $43(88 \%)$ & 49 \\
\hline Glra2/3 $^{-/}$ & $29(85 \%)$ & 34 & $41(87 \%)$ & 47 \\
\hline
\end{tabular}



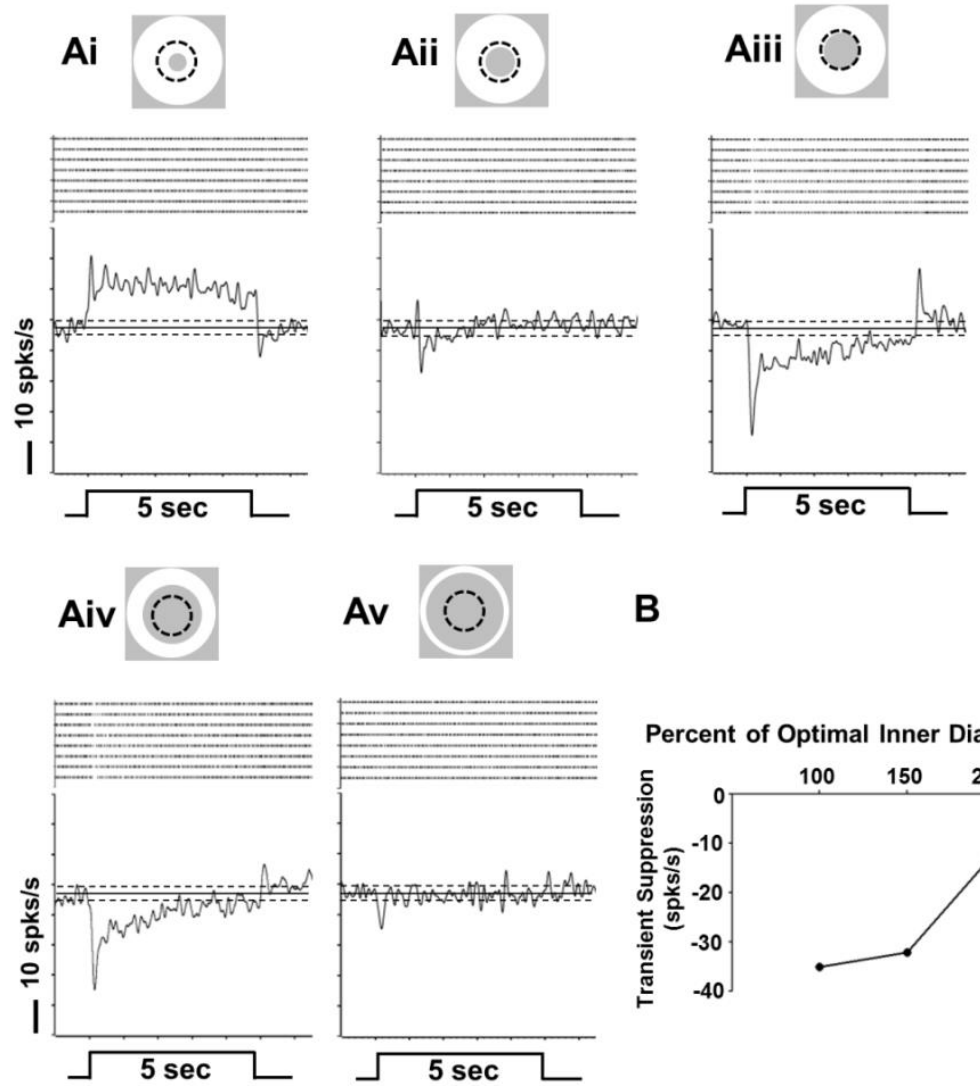

B
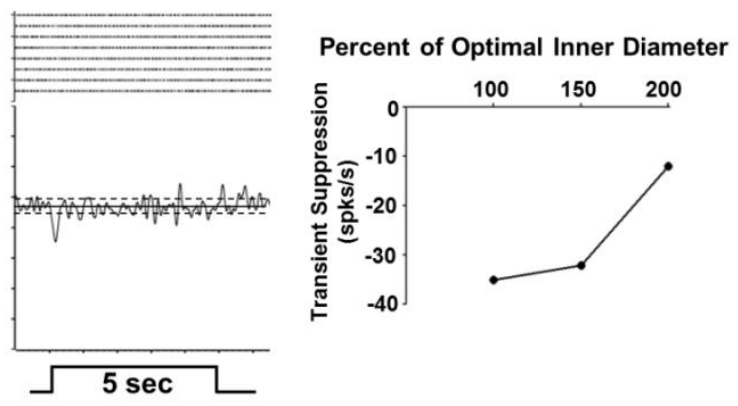

Figure 3.2. Representative PSTHs from an WT ON sustained RGC in the presence of bright annulus with varying inner diameter (ID). (Ai) An annulus with ID smaller than RF center (dotted circle) stimulates both RF center and surround and produces an excitatory response in the RGC (above mean spontaneous activity, SA). (Aii) With increased ID the annulus evokes a reduced excitation, which results from the balance between RF center excitation and surround suppression. (Aiii) When the annulus ID matches RF center and only stimulates RF surround the RGC response is suppressed (below the SA). (Aiv-v) The suppression changes as annulus ID gets larger and less RF surround is stimulated. (B) The transient suppression of the RGC in A as a function of ID indicated as a percentage of the optimal ID (100\%). 


\section{Isolated RF Suppression is Found in Most WT Sustained and Transient RGCs}

Annular stimulation evoked suppression in the majority $(51 / 56,91 \%)$ of WT ON RGCs (Figure 3.3A) and the transient response suppression occurred within $0-0.4 \mathrm{~s}$ of stimulus onset. The maintained suppression (0.4-5 s) of ON RGCs showed three temporal profiles (Table 3.2). In $43.1 \%$ of WT ON RGCs the duration of suppression matched the stimulus ( $5 \mathrm{~s}$ ) (Sustained; Figure 3.3Ai), indicating a maintained surround inhibitory input. In $27.5 \%$ of WT ON RGCs (Transient; Figure 3.3Aii) the duration of suppression was transient and returned to spontaneous activity levels $<4 \mathrm{~s}$ after stimulus onset, indicating a more transient surround inhibitory input. In $29.4 \%$ of WT ON RGCs (Mixed; Figure 3.3Aiii) the transient suppression was followed by a significant increase in excitatory activity above SA that occurred within $1 \mathrm{~s}$ of stimulus onset. The duration of this excitatory response varied between 0.5 and $5 \mathrm{~s}$. This indicates an interaction between a transient inhibitory input and a maintained excitatory input within the isolated surround.

Annular stimulation evoked suppression in the majority $(35 / 37,95 \%)$ of WT OFF RGCs (Figure 3.3B) and the transient response suppression also occurred within $0-0.4 \mathrm{~s}$ of stimulus onset. Their maintained suppression (0.4-5 s) showed two profiles (Table 3.3). In $20 \%$ of WT OFF RGCs the duration of suppression matched the stimulus (Figure 3.3Bi) and in the rest the duration of suppression was transient and returned to SA levels $<4 \mathrm{~s}$ after stimulus onset (Figure 3.3Bii). 
Table 3.2 Summary of ON RGCs with three different maintained response profiles across genotype

\begin{tabular}{l|c|ccl}
\hline & Total & Sustained & Transient & Mixed \\
\hline WT & 51 & $22(43.1 \%)$ & $14(27.5 \%)$ & $15(29.4 \%)$ \\
\hline GIra2 $^{-/}$ & 56 & $29(51.8 \%)$ & $22(39.3 \%)$ & $4(8.9 \%)$ \\
\hline GIra3 $^{-/}$ & 62 & $33(53.2 \%)$ & $15(24.2 \%)$ & $14(22.6 \%)$ \\
\hline GIra2/3/- $^{-/}$ & 29 & $11(37.9 \%)$ & $10(34.5 \%)$ & $8(27.6 \%)$ \\
\hline
\end{tabular}

Table 3.3 Summary of OFF RGCs with sustained and transient maintained response profiles across genotype.

\begin{tabular}{l|c|cc}
\hline & Total & Sustained & transient \\
\hline WT & 35 & $7(20.0 \%)$ & $28(80.0 \%)$ \\
\hline GIra2 $^{-/}$ & 51 & $3(5.9 \%)$ & $49(94.1 \%)$ \\
\hline GIra3 $^{-/}$ & 43 & $9(20.9 \%)$ & $34(79.1 \%)$ \\
\hline GIra2/3/ $^{-/}$ & 41 & $1(2.4 \%)$ & $40(97.6 \%)$ \\
\hline
\end{tabular}

ON and OFF WT RGCs have been separated into different classes based on the temporal nature of their RF center response into transient and sustained types (Cleland et al., 1971, 1973; Nobles et al., 2012). Published data have suggested that there are sustained and transient response channels that arise within the ON and OFF IPL sublaminae. However, comparisons between the 
temporal characteristics of the RF center and surround in mouse RGCs have not been made. I found that the majority of WT ON RGCs (48 of 51) in my sample had sustained RF center responses and only 3 ON RGCs were transient. Similarly, the majority of WT OFF RGCs (30 of 35) also had a sustained RF center response and only 5 were transient. This precluded a comparison of RF center and surround temporal characteristics among the transient ON and OFF RGCs. My comparison of WT ON and OFF RGCs with sustained RF center responses showed no correlation with surround temporal characteristics, the duration of the surround response varied widely (data not shown).

\section{Isolated RF Suppression is Found in Most Single and Double KO ON/OFF RGCs}

We previously noted differences in SA levels in Glra3 ${ }^{-/} \mathrm{ON}$ and $\mathrm{Glra2}^{-/-}$ OFF RGCs (Nobles et al., 2012). Similarly, I subtracted the mean SA from all responses prior to statistical comparisons.

Similar to WT, surround suppression was found in almost all Glra2 ${ }^{-/}$, Glra3 $^{-/}$and Glra2/3/ ON and OFF RGCs (Figure $3.3 \&$ 3.5). The percentage of RGCs with surround suppression was large and similar across genotype (Table 3.1; Chi-square, ON, $p=0.27$; OFF, $p=0.57$ ). At least one annulus ID evoked response suppression significantly below SA levels in all KO RGCs. As in WT, the RF center diameter of the KO RGCs that lacked a response suppression was larger than widest inner diameter produced on our computer display. Regardless of center sign, part of the KO RGCs were examined with two annulus IDs greater than the optimal (Glra2 ${ }^{-/}$, ON 62\%, OFF 59\%; Glra3 $3^{-/}$, ON 66\%, OFF 86\%; 
Glra2/3/\%, ON 55\%, OFF 68\%). All RGCs with significant surround suppression were included in my quantitative analyses of optimal RF surround suppression and comparisons across genotypes; except that the RGCs with a minimum firing rate equals to 0 spikes/s were excluded from comparisons of transient suppression to avoid underestimating the magnitude. Only RGCs examined under at least two annulus IDs greater than the optimal were included for comparisons across annulus ID.

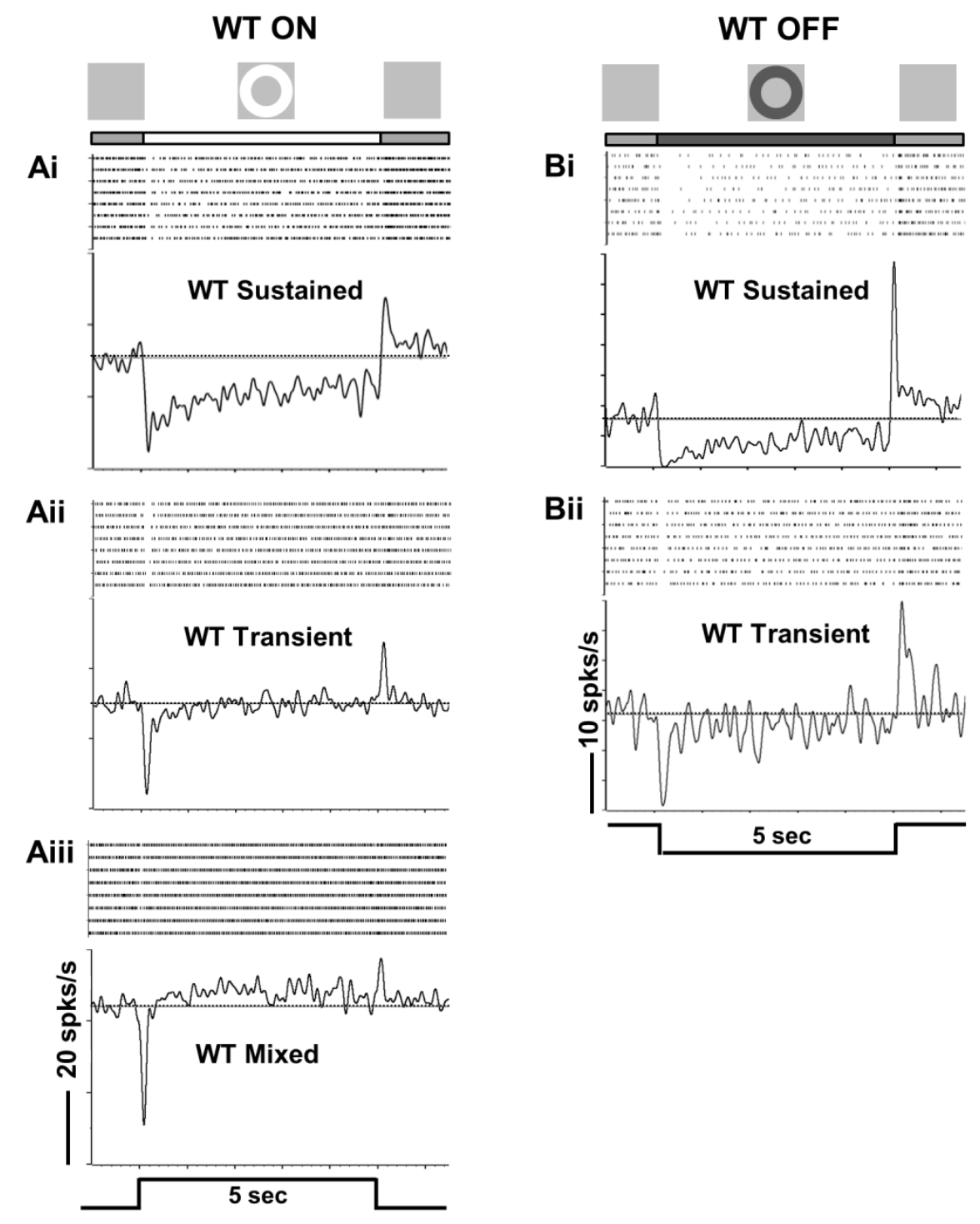


Figure 3.3. RF optimal surround suppressive responses of WT ON and OFF RGCs. Average PSTHs for ON and OFF RGCs evoked by their optimal annulus are shown along with raster plots (above) that show responses to individual presentations of the stimulus. The stimulus profile (5 second duration) is shown at the bottom of each column. A bright annulus $\left(100 \mathrm{~cd} / \mathrm{m}^{2}\right)$ or dark annulus $\left(0 \mathrm{~cd} / \mathrm{m}^{2}\right)$ was presented on a screen whose average luminance was $\left(20 \mathrm{~cd} / \mathrm{m}^{2}\right)$. (Ai \& Bi) The spiking activities of ON and OFF Sustained RGCs are significantly decreased compared to mean spontaneous activity (SA, solid line) in the presence of a bright annulus for the whole stimulation (5s). (Aii \& Bii) The spiking activities of ON and OFF Transient RGCs are significantly decreased to below mean SA at stimulus onset and returns to SA level before stimulus offset. (Aiii) The spiking activity of ON Mixed RGCs is significantly decreased to below mean SA at stimulus onset and increased to above SA level before the stimulation offset. 
GlyR KO ON

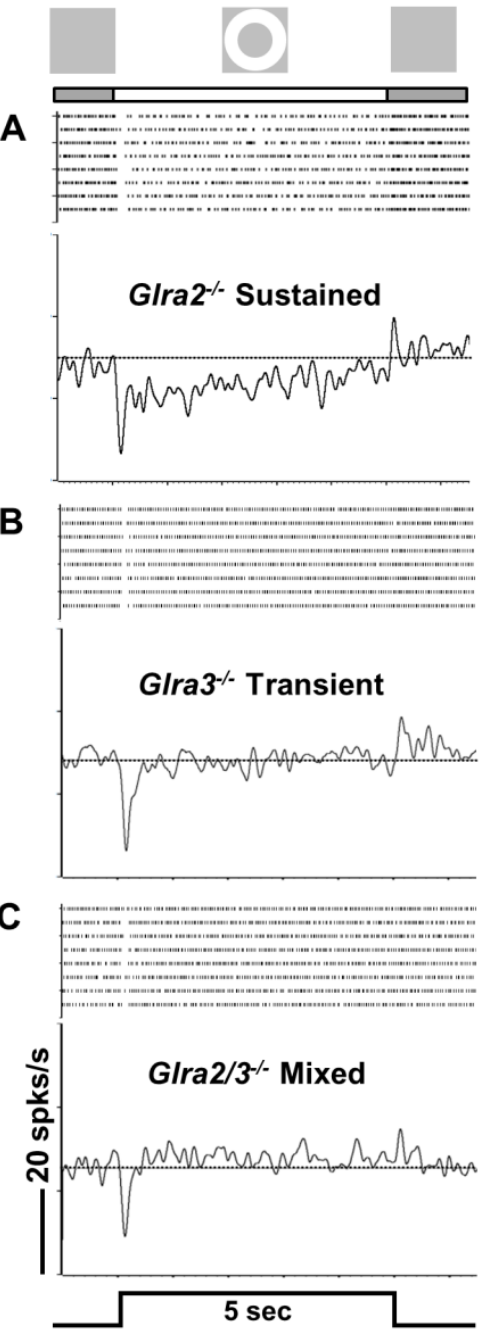

GlyR KO OFF
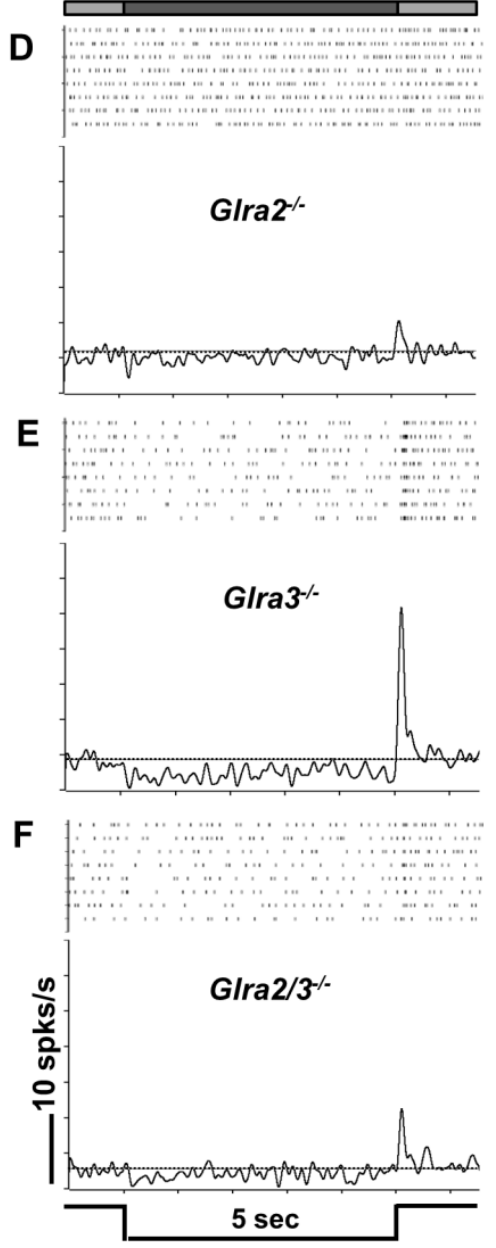

Figure 3.4. RF optimal surround suppressive responses of GlyR KO ON and OFF RGCs. Average PSTHs for ON and OFF RGCs evoked by their optimal annulus are shown along with raster plots (above) representing responses to individual presentations of the stimulus. The stimulus profile (5 second duration) is shown at the bottom of each column. A bright annulus $\left(100 \mathrm{~cd} / \mathrm{m}^{2}\right)$ or dark annulus $\left(3 \mathrm{~cd} / \mathrm{m}^{2}\right)$ was presented on a screen whose average luminance was (20 $\mathrm{cd} / \mathrm{m}^{2}$ ). (A-C) Representative ON RGCs with three different maintained response 
profiles in Glra2 ${ }^{-/}$Glra3 ${ }^{-/}$, and Glra2/3/- retina. (D-F) Representative OFF RGCs in Glra2 ${ }^{-/}$Glra3 ${ }^{-/}$, and Glra2/3/- retina. The mean spontaneous activity of each RGC is indicated with a solid line.

\section{Neither GlyRa2 nor GlyRa3 Modulates the RF Surround of WT ON RGCs}

Similar to WT, I found the same maintained surround suppression profiles in ON RGCs across Glra2 ${ }^{-/}$, Glra3 ${ }^{-/}$and Glra2/3/- and in numbers equivalent to WT (Chi-square; $p=0.07$ ). This result indicated that the absence of GlyRa2 or GlyRa3 did not contribute to the ON RGC RF surround suppression.

In ON Sustained, ON Transient and ON Mixed RGCs, transient suppression were similar between each single KO ( $\mathrm{Glra}^{-/}$and $\left.\mathrm{Glra3}^{-/}\right)$and WT, or and double KO $\left(\right.$ Glra2 $\left./ 3^{-/}\right)$at the optimal ID (Figure 3.5A) and at all larger IDs (data not shown). Similarly, there also were no differences in maintained suppression (0.4-5s) of ON sustained RGCs across these genotype at the optimal ID (Figure 3.5B) and at all larger IDs (data not shown). Taken together, my results suggest that neither GlyRa2 nor GlyRa3 contribute to surround suppression in the input pathways to WT ON RGCs. 
Ai
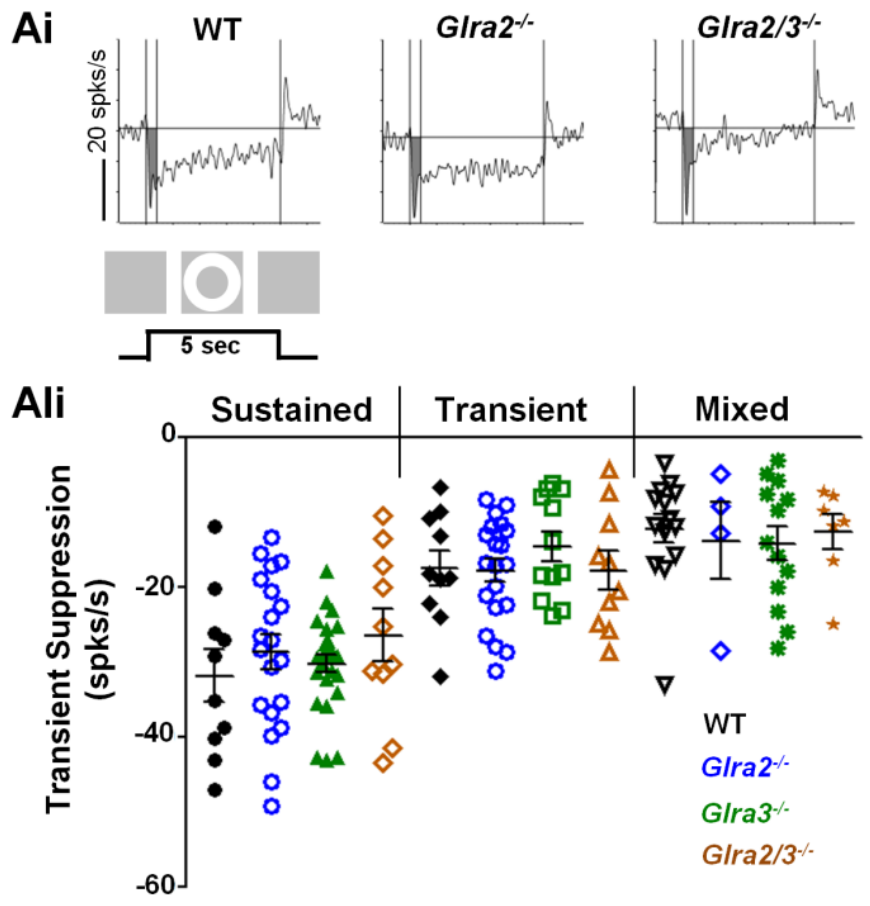

$\mathrm{Bi}$
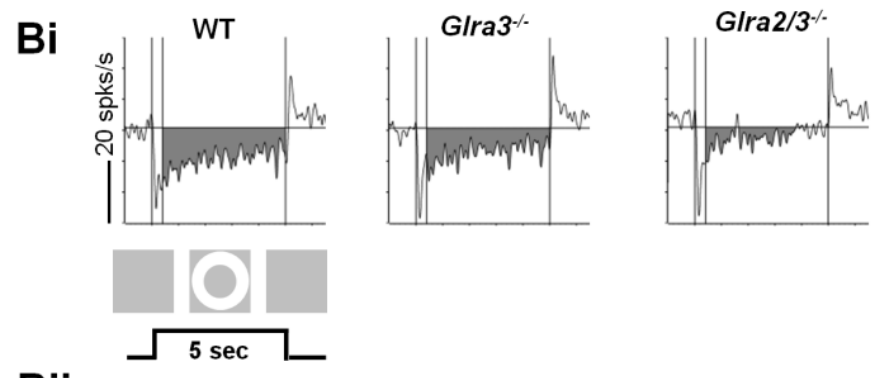

$\mathrm{Bii}$

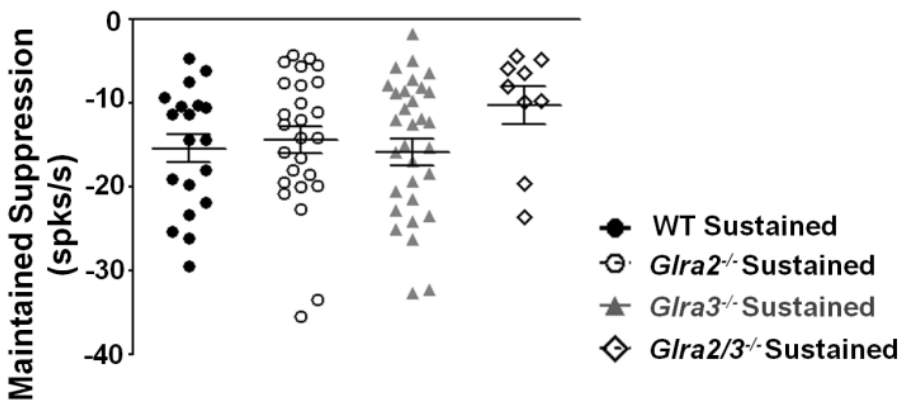

Figure 3.5. GlyRa2- and GlyRa3 do not contribute to the receptive field surround suppression of ON RGCs. (Ai, Bi) Representative PSTHs of WT, single and double KO ON RGCs during stimulation of receptive field surround with bright annulus. (Ai) Shaded regions represent the transient $(0-0.4 \mathrm{~s})$ 
response component and $\mathbf{( B i )}$ the maintained response component (0.4-5s). (Aii) The scatter plot shows the distribution of the transient suppression at optimal ID, which is similar across genotype and groups. (Bii) The maintained suppression in the Sustained group is similar across genotype.

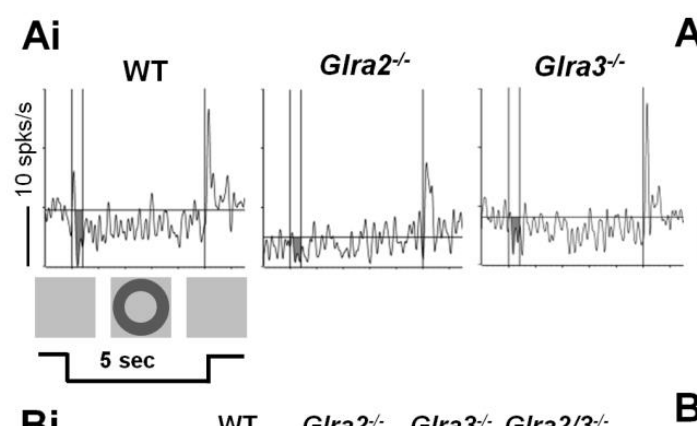

Aii

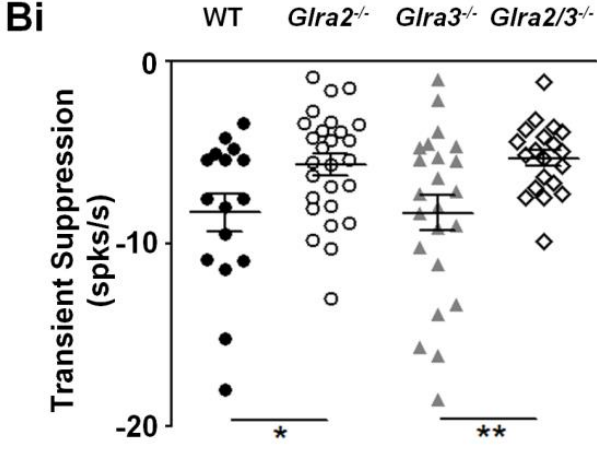

Bii Percent of Optimal Inner Diameter

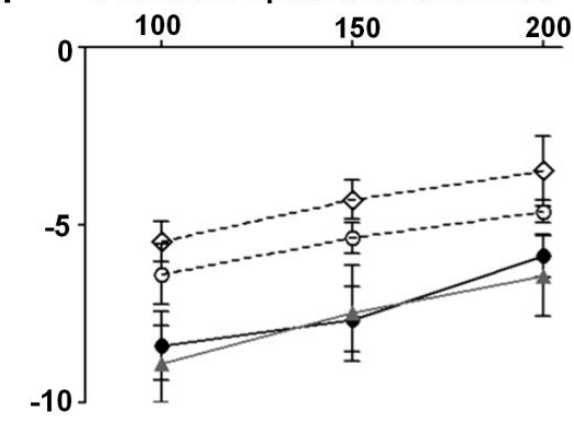

Ci

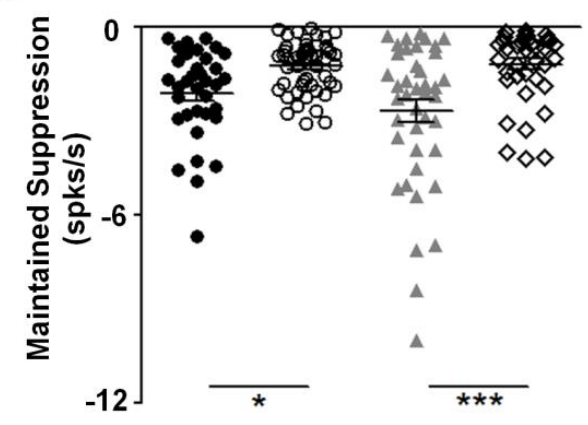

Cii
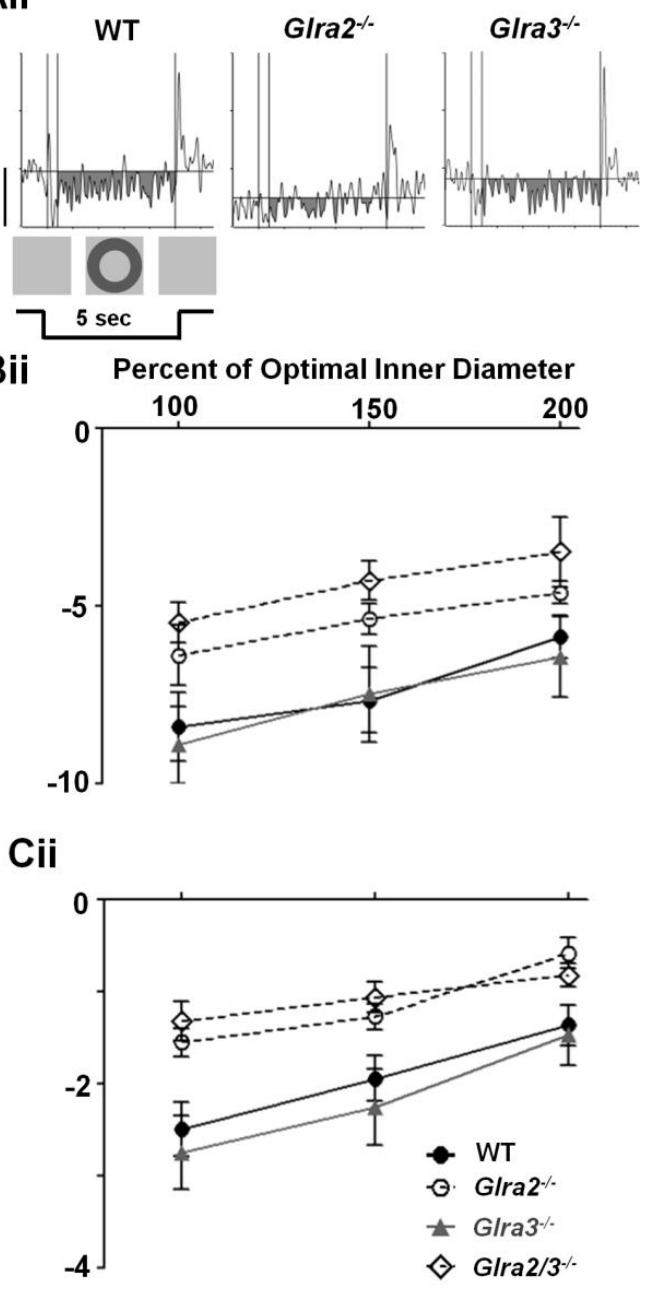

Figure 3.6. Only GlyRa2-mediated inhibition modulates the receptive field suppression of OFF RGCs. (Ai-ii) Representative PSTHs of WT, GIra2 ${ }^{-/}$and Glra3 ${ }^{-/}$RGCs during stimulation of receptive field surround with dark $\left(3 \mathrm{~cd} / \mathrm{m}^{2}\right)$ annulus. (Ai) Shaded regions represent the transient (0-0.4s) response 
component and (Aii) the maintained response component (0.4-5s). (Bi) The scatter plot shows the distribution of the transient suppression and the differences across genotype. The transient suppression of Glra2 ${ }^{-/}$(open circles, $\mathrm{n}=27$ ) is lower than WT (closed circles, $\mathrm{n}=16$ ) while Glra3 ${ }^{-/}$OFF GCs (grey triangles, $\mathrm{n}=23$ ) are similar to WT. Glra2/3/- OFF GCs (open diamonds, $\mathrm{n}=20$ ) are lower compared to Glra3 ${ }^{-/}$but similar with Glra2 ${ }^{-/}$. (Bii) The mean transient suppression across annulus ID for each genotype (WT, $n=12$; Glra2 $2^{-/}, n=14$; ,

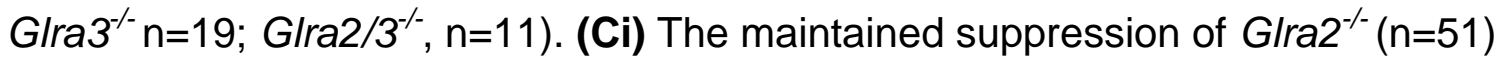
is significantly lower than WT $(n=35)$ while Glra3 ${ }^{-/-}$OFF GCs $(n=43)$ are similar to WT. GIra2/3/- OFF GCs $(n=41)$ are significantly lower compared to G/ra3 ${ }^{-/-}$but similar to Glra2 $^{-/}$. (Cii) The mean maintained suppression across annulus ID for

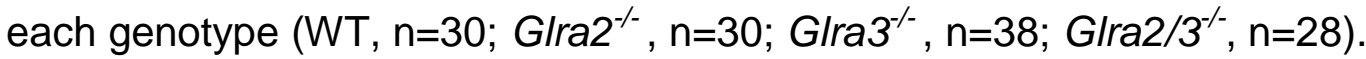

\section{Only GlyRa2 Modulates RF Surround Suppression in OFF RGCs}

In contrast to ON RGCs, there were fewer sustained OFF RGCs in GIra2 ${ }^{-1-}$ compared to WT and fewer Glra2/3/- OFF RGCs compared to Glra3 ${ }^{-/}$(Table 3.3; Chi-square, $p=0.01$ ). In our previous study, we found that the absence of either GlyRa2 or GlyRa3 subunit expression significantly altered the temporal nature of RGC RF center response (Nobles et al., 2012). This precluded the separation of OFF RGCs into sustained/transient types. 
At the optimal and larger annular IDs, both the transient and maintained suppression in Glra3 ${ }^{-/-}$and WT OFF RGCs were similar (Figure 3.6Bi, Ci) and this also was true of the duration of their surround suppression $(2.2 \pm 0.3 \mathrm{vs}$. $2.0 \pm 0.3 \mathrm{~s})$. These results suggest that GlyRa3 input does not contribute to the surround suppression in our sample of OFF RGCs.

In contrast, all aspects of the RF surround suppression differed when GIra2 ${ }^{-/}$OFF RGCs were compared to WT. GIra2 $2^{-/}$OFF RGCs had significantly less transient and maintained surround suppression at their optimal ID compared to WT (Figure 3.6Bi; transient, $p=0.02$; Figure 3.6Ci; maintained, $p=0.005$ ). These differences persisted as the annulus ID increased (Figure 3.6Cii; Two-way ANOVA; transient, $p<0.0001$; sustained, $p<0.0001)$. I found similar changes in the transient and maintained surround suppression at all IDs $\geq$ optimal ID in Glra2/3/\% OFF RGCs compared to Glra3- (Figure 3.6B; Two-way ANOVA, transient, $p<0.0001$; Figure 3.6C; maintained, $p<0.0001$ ). Consistent with less maintained surround suppression, the duration of suppression was significantly shorter in G/ra2 ${ }^{-/}$OFF RGCs $(1.5 \pm 0.2 \mathrm{~s} ; p=0.03)$ compared to WT, and in Glra2/3/- OFF RGCs $(1.0 \pm 0.2 \mathrm{~s} ; p=0.0004)$ compared to Glra3 ${ }^{-/}$.

Together with our previous results (Nobles et al., 2012), my results suggest that GlyRa2 mediated inhibition enhances both RF center excitation and RF surround suppression in the WT Off pathway. They also show that GlyRa3 mediated inhibition is isolated to the WT On pathway to enhance RF center excitation but has no effects in the WT Off pathway. Two circuits in the WT retina are proposed to explain the changes between the G/ra2 ${ }^{-/}$and WT OFF RGCs. 
One is an inhibitory pathway that uses a crossover-feedforward mechanism and the other a concatenated circuit (Figure 3.11). These are described and their likelihoods evaluated in the Discussion.

\section{Post-stimulus Excitation in OFF but not ON RGCs is Altered by the Absence of GlyRa2 Expression}

The offset of an annulus evokes a post-stimulus excitation in both WT ON and OFF RGCs, regardless of whether suppression is sustained or transient (Figure 3.7A). I found that the post-stimulus peak amplitude of both WT ON and OFF RGCs was significantly correlated with their transient suppression (Figure 3.7B; ON, $r=-0.56, p<0.0001 ;$ OFF, $r=-0.58, p=0.0003)$. This suggests that the RF surround suppression and post-stimulus excitation in RGCs may be subject to the same modulation (see discussion).

Consistent with this hypothesis, there was no significant difference in the post-stimulus peak amplitude in ON RGCs across genotype (Figure 3.7Ci; WT,

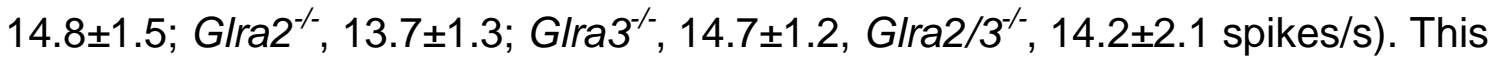
bolsters my conclusion that neither GlyRa2 nor GlyRa3 contribute to ON RGC surrounds. Further, the peak amplitude of post-stimulus excitation in Glra3 ${ }^{-/}$OFF RGCs (34.8 \pm 4.8 spikes/s) also was similar to WT (26.6 \pm 3.0 spikes/s).

In contrast, the peak amplitude of post-stimulus excitation was smaller in Glra2 ${ }^{-/}$OFF RGCs (21.2 \pm 2.8 spikes/s) compared to WT (Figure 3.7Bii; $p=0.04$ ),

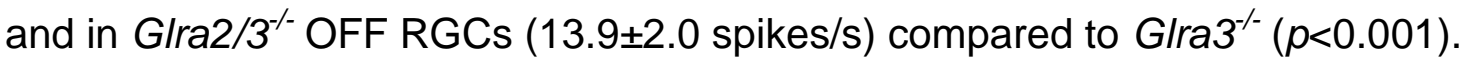
Again, these results are consistent with other aspects of the response evoked by 
annular stimuli and reinforces my hypothesis of a selective role for GlyRa2mediated inhibition to the OFF RGC RF surround. I propose that the level of post-stimulus excitation could be related to several mechanisms that require GlyRa2 mediated inhibition (see discussion).

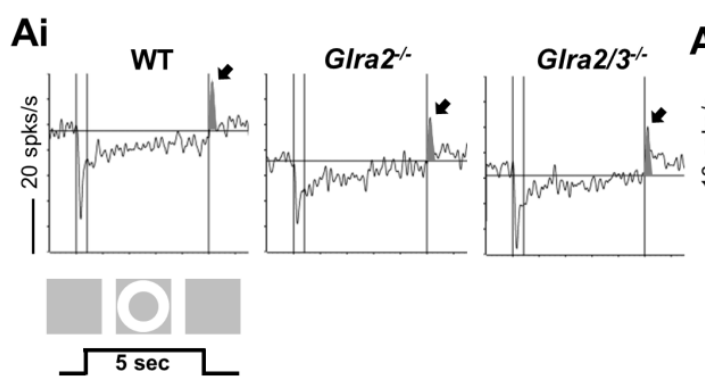

$\mathrm{Bi}$
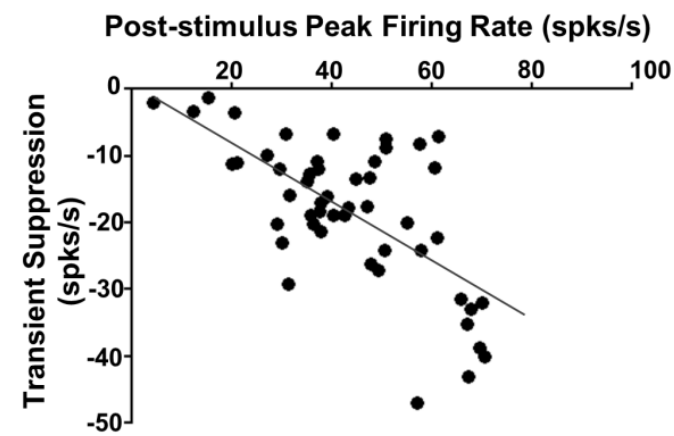

$\mathbf{C i}$

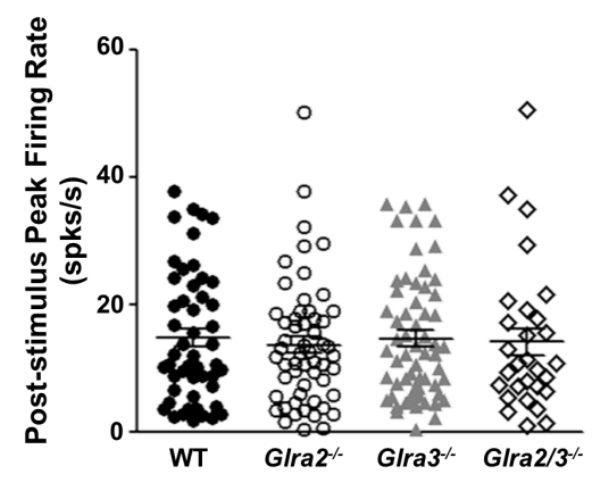

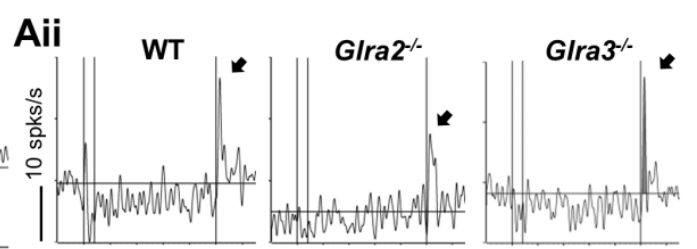

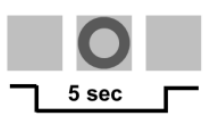

$\mathrm{Bii}$

WT OFF RGCs Post-stimulus Peak Firing Rate (spks/s)

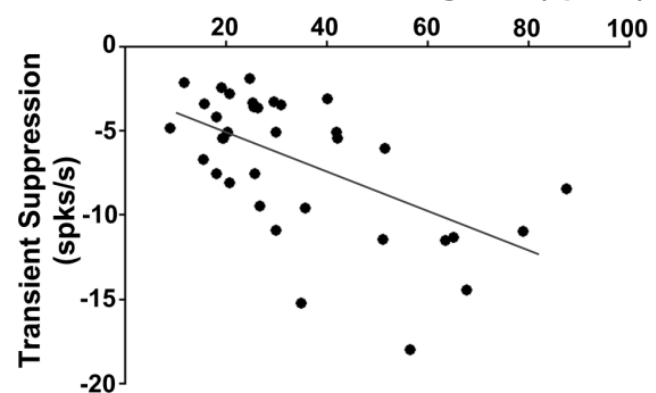

Cii

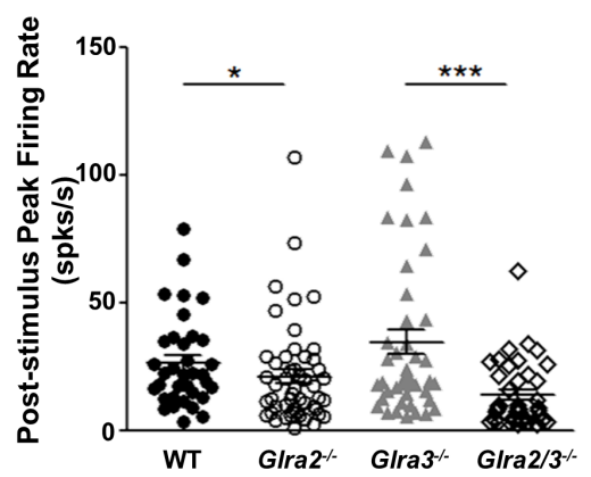

Figure 3.7. The post-stimulus excitatory peak amplitude of only OFF RGC is affected by the absence of GlyRa2 expression. (A) Representative PSTHs of WT, GIra2 ${ }^{-/}$and Glra3 ${ }^{-/}$ON (Ai) and OFF (Aii) RGCs receptive field surround 
suppression. Post-stimulus peak amplitudes are indicated with black arrows. (B) In both WT ON (Bi, $n=51)$ and OFF (Bii, $n=35)$ RGCs, the transient suppression during stimulation is significantly correlated with the post-stimulus peak amplitude. (C) The scatter plot shows the distribution and the differences of the poststimulus peak amplitude across genotype. (Ci) The post-stimulus peak amplitude of ON RGCs is similar across genotype (WT, closed circles, $n=51$; Glra2 ${ }^{-/}$, open circles, $n=56$; Glra3 ${ }^{-/}$, grey triangles, $n=62 ;$ Glra2 $/ 3^{-/}$, open diamonds, $n=28$ ). (Cii) The post-stimulus peak amplitude of Glra2 ${ }^{-/}(n=51)$ OFF RGCs is smaller compared to WT $(n=35)$ but similar to Glra2/3/- OFF RGCs $(n=40)$. The poststimulus peak amplitude of Glra3 ${ }^{-/}(n=43)$ OFF RGCs is similar to WT but larger compared to Glra2/3/- OFF RGCs.

\section{GlyR Subunit-specific Inhibition Alters the Balance of RF Excitation and Suppression in the On but not Off Pathway.}

The spatial organization of the RF center/surround, their interactions and overall sensitivity has been mathematically modeled as a difference of Gaussians (Figure 3.8; Rodieck and Stone, 1965). The RF center is modeled by one Gaussian excitatory input. The surround is modeled by another Gaussian inhibitory input, but include both local inhibition continuous with the RF center as well as more wide spread lateral inhibition. The interaction between the RGC RF center and surround is critical for temporal and spatial vision. Previously, we 
established roles for GlyRa2 and GlyRa3 in the RF center response (Nobles et al., 2012), which reflects the combined center excitation and local inhibition. Both GlyRa2 and GlyRa3 mediate inhibition that enhances the RF center response in the On pathway, whereas only GlyRa2 mediates inhibition that enhances the RF center responses in the Off pathway. In my study of the contributions of GlyRa2 and GlyRa3 in the isolated RF surround response, I find that only GlyRa2 mediates inhibition that enhances to the RF surround and only in the Off pathway.

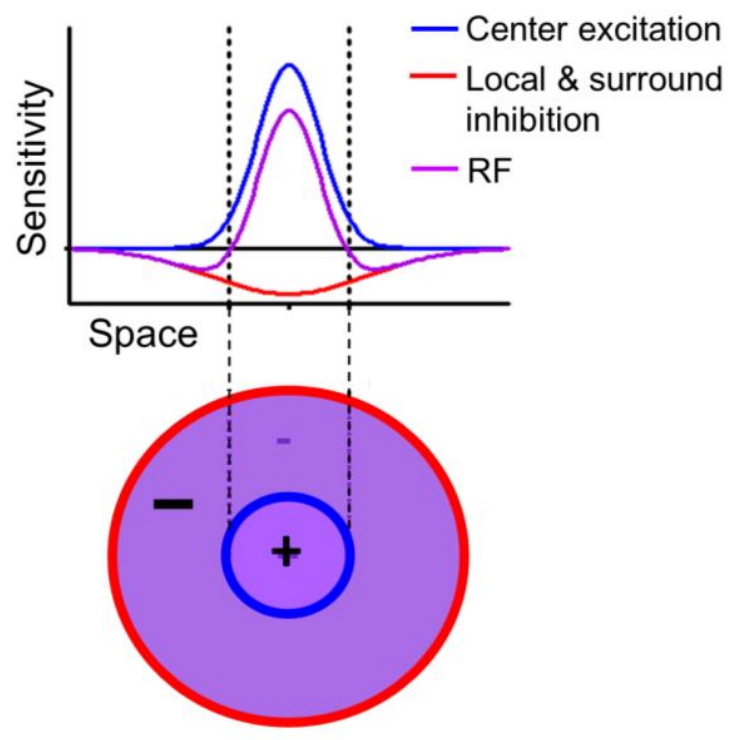

Figure 3.8. A Difference of Gaussians model for RF center/surround spatial organization. The spatial organization of a RGC receptive field has been represented by two co-extensive mechanisms with different sensitivity profiles that sum together to create a response profile (purple) for each individual RGC. The RF center excitation (blue) with local RF surround (red) creates the RF center $(+)$ roughly matching the dendritic span of RGC. The area of the RF 
surround (-) extends over larger regions of the retina outside the span of the dendrites and is dominated by inhibition (red) (modified from Rodieck and Stone, 1965).

If my results and interpretation are correct, certain predictions can be tested about changes in the balance between the RF center and surround, a metric I define as the balanced-response ID, the annulus diameter where RGCs receive equal excitatory and inhibitory inputs across stimulated RF (Figure 3.9A). A smaller center:surround ratio requires a smaller ID to recruit more center excitation to balance the surround inhibition (Figure 3.9B).

The predictions are:

1. The absence of GlyRa2 or/and GlyRa3 expression reduce the RF center sensitivity but not the surround sensitivity and neither change the sign of RF center or surround (Figure 3.9B). Therefore, this should result in a smaller center:surround ratio and balanced-response ID in all KO ON RGCs.

2. The absence of GlyRa2 expression reduces both RF center and surround (Figure 3.9C) and GlyRa3 does not play a role in the Off pathway. Therefore, this should result in unaltered center:surround ratio and balanced-response ID in all KO OFF RGCs. 
To test these predictions, I compared the balanced-response IDs of RGCs across RF center class and genotype. To determine the balanced-response ID, I plotted the transient peak/minimum firing rate of each cell as a function of annulus ID, and determined the ID where the firing rate equals mean SA (Figure 3.10A). As predicted, the absence of either GlyRa2 or GlyRa3 expression resulted in a significantly smaller balanced-response ID in ON RGCs compared to WT (Figure 3.10B; WT, 829.2 \pm 62.6 ; Glra2 ${ }^{-/}, 606.0 \pm 36.48 ; p=0.002$; Glra3 $^{-/}$, $663.0 \pm 57.13 \mu \mathrm{m} ; p=0.02)$. The ID of Glra2/3 ${ }^{-/}$ON RGCs $(558.5 \pm 59.84 \mu \mathrm{m})$ was similar to the ID of both single KOs. In contrast, the ID of OFF RGCs was similar across all genotypes (Figure 3.10C; WT, 577.9 \pm 51.7 ; Glra2 $^{-/}, 679.1 \pm 60.37$; Glra3 $^{-/}, 485.8 \pm 41.24 ;$ Glra2/3 $\left.{ }^{-/}, 556.0 \pm 63.3 \mu \mathrm{m}\right)$. These results support my prediction of center:surround ratio and previous conclusion that the pattern of glycinergic modulation differs between the On and Off pathway.

Together with our previous results, my data define the contributions of two GlyR a subunits on RF center/surround responses. The results show a consistent picture, a shared, but independent contribution from GlyRa2 and GlyRa3 in the On pathway that is confined to the RF center. They also show a selective role of GlyRa2 in the Off pathway that contributes to both the RF center and surround. This suggests that glycinergic input is both local and lateral in the Off pathway. 


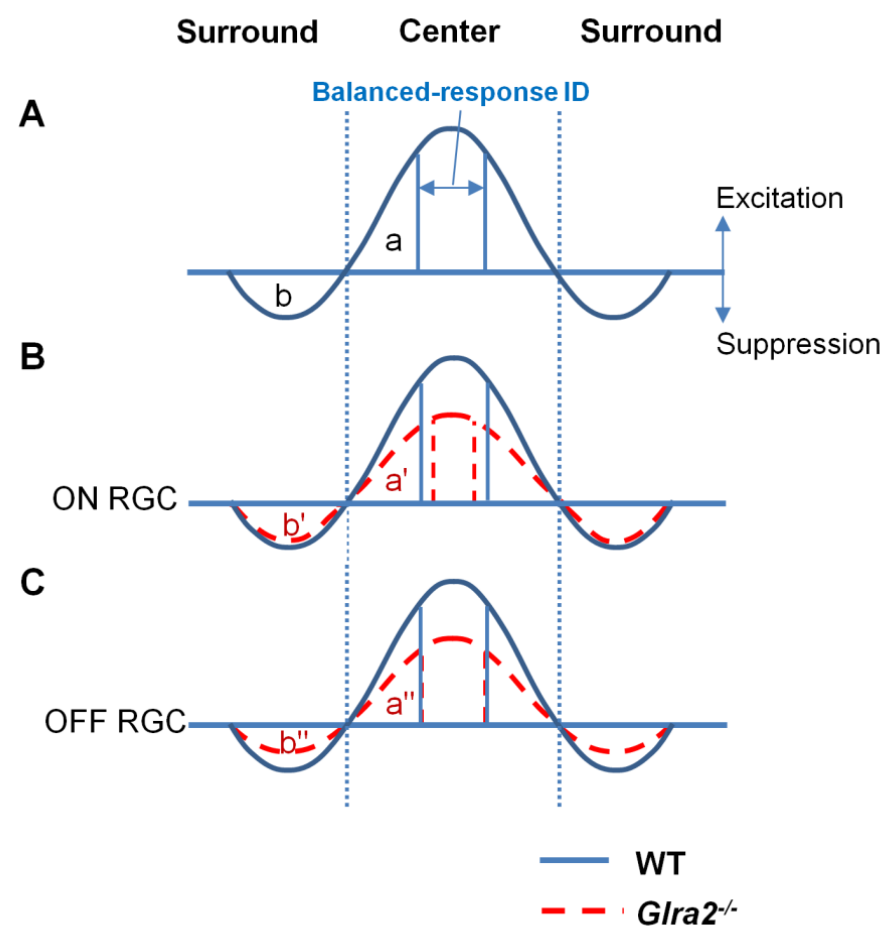

Figure 3.9. A model of altered balanced-response inner diameter of ON but not OFF RGCs. (A) Each RGC has an excitatory center (outward curve) and a suppressive surround (inward curve). An inner diameter (ID) which gives equal size of area $a$ and $b$ is considered as balanced-response ID, evoking same amount of center excitation and surround suppression. (B) Compared with WT ON RGCs (blue line), GIra2 ${ }^{-/}$ON GCs (red dash line) have significantly decreased center excitation and unaltered surround suppression. As a result, a smaller annulus ID is required to reach center-surround balance, where area a' equals to area b'. (C) In GIra2 ${ }^{-/}$OFF RGCs (red dash line), both center excitation and surround suppression are significantly less compared to WT OFF RGCs (blue line), presumably with similar extent (same amount of decrease in size of 
area $a$ and $b)$. As a result, the balanced-response ID stays the same where area a" equals to area b".
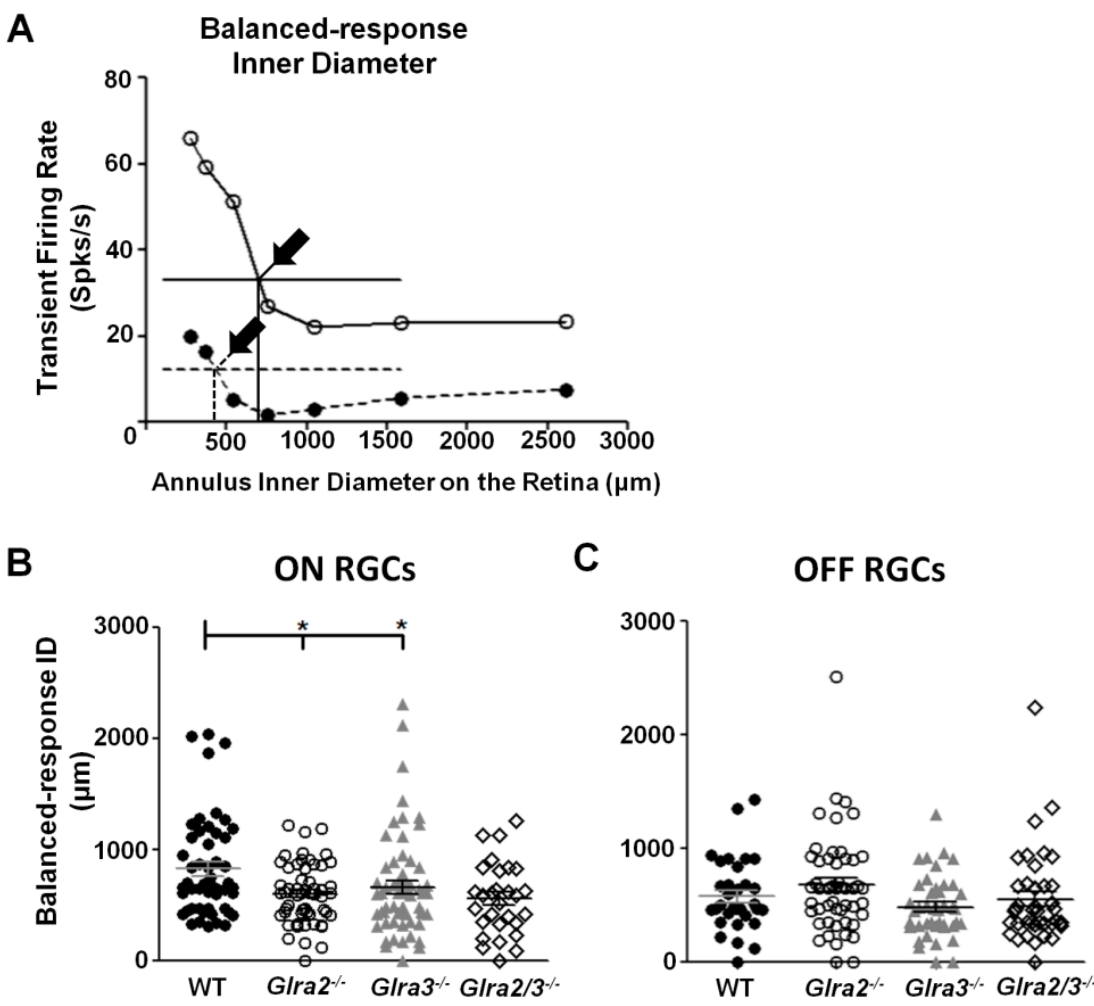

Figure 3.10. The absence of GlyRa2 or GlyRa3 alters the balancedresponse inner diameter of ON but not OFF RGCs. (A) Transient annulus response function for representative WT ON and OFF RGCs. Transient firing rates above SA (straight lines) are the peak amplitudes of RGCs at stimulus onset, while transient firing rate below $S A$ are the minimum firing rates of RGCs at stimulus onset. The balanced-response IDs are indicated by arrows where the transient firing rate equals to SA. (B\&C) The scatter plot shows the distribution of the balanced-response inner diameter and the differences across genotype. (B) ON RGCs. The balanced-response inner diameter of both G/ra2 ${ }^{-/}$(open circles, 
$\mathrm{n}=56$ ) and Glra3 $^{-/}$(grey triangles, $\mathrm{n}=62$ ) are smaller compared to WT ON RGCs (closed circles, $\mathrm{n}=51$ ). Glra2/3 ${ }^{-/}$ON RGCs (open diamonds, $\mathrm{n}=28$ ) are similar with both single KOs. (C) OFF GCs. The balanced-response inner diameter of both Glra2 $^{-/}(n=51)$ and Glra3 $^{-/}(n=43)$ are similar to WT OFF RGCs $(n=35)$. Glra2/3/-OFF RGCs $(n=40)$ are similar to both single KOs.

\section{Discussion}

\section{Glycinergic Inhibition within IPL Modulates Surround Antagonism of OFF RGCs at Photopic Light Level}

Previous studies across a number of species indicate that $\mathrm{GABA}_{\mathrm{A}} \mathrm{R}$ mediated feedforward and $G A B A_{C} R$-mediated feedback inhibition within the inner retina shapes surround antagonism of RGCs (Buldyrev and Taylor, 2013; Cook and McReynolds, 1998; Daw et al., 1990; Flores-Herr et al., 2001; Kirby and Enroth-Cugell, 1976; O’Brien et al., 2003; Roska et al., 2000). Although we and others demonstrate a role for glycinergic input in shaping the RF center, the role of glycinergic inhibition to RF surround has not been directly observed, although there is evidence for this idea in the published literature. Strychnine, an antagonist of all GlyR subunits, attenuates the RF surround response of ON and OFF X-type cat RGCs (Kirby, 1979). A similar change, the absence of surround suppression, also was observed in the ON, OFF and ON-OFF RGCs in the spastic mouse that harbors a mutation in the gene coding GlyR $\beta$ subunit, which

significantly reduces overall synaptic glycinergic transmission (Stone and Pinto, 
1992). My study addresses the role of specific GlyR subunits (GlyRa2 and GlyRa3) in modulating RGC RF surround. Using extracellular recordings of RGCs in vivo and annular stimuli to isolate the RF surround component, I compared WT RGC responses with those in Glra2 ${ }^{-/}$, Glra3 ${ }^{-/}$and Glra2/3\% mice. The use of KO mice can be considered as a substitute for a subunit selective glycine antagonist. My results show that in the WT ON cone pathways neither GlyRa2 nor GlyRa3 inhibition contribute to surround suppression. My results also show that in the OFF cone pathway only GlyRa2 inhibition contributes to surround suppression. Moreover, my results argue that the subunit specific contribution of glycinergic modulation of RF center vs. surround differs between the On and Off retinal pathway.

\section{Mechanisms That Contribute to Post Stimulus Rebound Excitation in WT OFF RGCs at Luminance Decrement}

Mechanisms that generate spontaneous activity in WT RGCs have been shown to include: intrinsically generated spontaneous activity of some WT OFF RGCs and modulation by synaptic input (Margolis and Detwiler, 2007). In the same WT OFF RGCs, rebound excitation, another intrinsic property, can be evoked by application of negative current in the absence of synaptic input and a role for low-voltage gated (LVA) $\mathrm{Ca}^{2+}$ channels has been proposed (Margolis et al., 2010). I observed significantly less post-stimulus excitation in Glra2 ${ }^{-/}$OFF RGCs. Although I did not measure the currents that underlie the altered poststimulus excitatory response in the absence of GlyRa2 mediated inhibition, there are three likely mechanisms. 
1. In the WT retina, the spontaneous activity is modulated by GlyRa2mediated inputs that inhibit either a direct tonic inhibitory input onto the OFF RGC or onto their OFF cone BC excitatory inputs. A lower spontaneous rate is observed in G/ra2 ${ }^{-/}$OFF RGCs compared to WT (Nobles et al., 2012). This could result from absence of GlyRa2-mediated inhibition of a tonic inhibitory input onto either the OFF RGC or its presynaptic OFF BC. In either scenario, the tonic inhibition onto the Off pathway is increased, and the resting membrane potential of OFF RGCs should be more hyperpolarized. If the excitatory input remains unchanged, the amplitude of the post-stimulus excitation will be lower.

2. Alternatively, in WT OFF RGCs I found that the excitatory drive of the post-stimulus excitation was positively related to the suppression during stimulation, which is decreased in the GIra2 ${ }^{-/}$OFF RGCs. The weaker surround suppression evoked by the annular stimulus in Glra2 ${ }^{-/}$OFF RGC could compromise the de-inactivation of LVA Ca ${ }^{2+}$ channels. In this case, at stimulus offset (light increment), the RGCs receive less calcium influx and have their spiking activity reduced.

3. In the WT retina, a GlyRa2-mediated crossover inhibition could be involved to disinhibit WT OFF RGCs at the post-stimulus and in turn enhance the rebound excitation. Such a crossover inhibitory circuit should be absent in Glra2 ${ }^{-/}$. This crossover circuit is described in the following section (Figure 3.11). 


\section{A crossover-feedforward GlyRa2-mediated Circuit Enhances OFF RGC Surround Inhibition and Rebound Excitation}

In the absence of GlyRa2 subunit expression, OFF RGCs have weaker surround suppression compared to WT. This is unlikely due to the absence of GlyRa2-mediated inhibition directly onto OFF RGCs or their presynaptic BCs, because: 1) the surround antagonism of OFF RGCs has been observed to be generated by GABAergic inhibition from wide-field ACs (Flores-Herr et al., 2001;

O'Brien et al., 2003); 2) OFF BCs only express GlyRa1 (Ivanova et al., 2006) and 3) to date many OFF RGC types do not express GlyRa2 (Majumdar et al., 2007; Chapter V). Therefore, I propose that GlyRa2 are expressed on ACs where they mediate AC-to-AC inhibition (Majumdar et al., 2009; Weiss et al., 2008), which would modulate RGC surround antagonism indirectly. The rebound excitation at luminance increment evoked by RF surround stimulation of G/ra2 ${ }^{-/}$OFF RGCs also is smaller compared to WT. This argues that there is likely to be a GlyRa2mediated crossover-feedforward circuit that modulates the OFF RGC (Figure 3.11A). The crossover inhibition functions as a 'push-pull' mechanism to enhance the OFF RGC RF surround suppression by inhibition and its post-stimulus excitatory response by disinhibition. Glycinergic On-to-Off pathway crossover inhibition has been observed in BCs, ACs and RGCs where it fine tunes their visual responses (Hsueh et al., 2008; Manookin et al., 2008; Molnar et al. 2009; Murphy and Rieke, 2006). In addition, I show a similar GlyRa1-mediated circuit contributes to signaling in the PV5 OFF RGC (Zhang et al., 2014 and Chapter IV). 
In the proposed WT crossover-feedforward circuit, the suppressive surround of the OFF RGC is generated by an OFF GABAergic AC, which expresses GlyRa2 (Figure 3.11A). This GABAergic AC is postsynaptic to a bistratified glycinergic AC driven by the On pathway (crossover inhibition). Stimulus onset (luminance decrement within the RF surround of OFF RGCs) hyperpolarizes the presynaptic ON cone BCs (Figure 3.11A, box 1) and reduces/eliminates glycinergic inhibition from the bistratified AC onto the GABAergic AC (Figure 3.11A, box 2i). This increases the GABAergic input onto the OFF RGC and/or its presynaptic OFF BC and enhances its surround suppression (Figure 3.11A, box $3 \mathrm{i} \& 5 \mathrm{i}$ ). Stimulus offset (luminance increment) depolarizes the presynaptic ON cone BC (Figure 3.11A, box 1) and increases the glycinergic inhibition onto the GABAergic AC (Figure 3.11A, box 2i). The decreased output of the GABAergic AC (disinhibition) facilitates excitation in the OFF RGC (Figure 3.11A, box3i \& 5i). In the absence of GlyRa2 expression, GlyRa2-mediated crossover 'push-pull' modulation of the GABAergic AC is eliminated (Figure 3.11A, box2ii \& 3ii) and with it the enhancement of surround suppression at luminance decrement as well as post-stimulus rebound excitation at luminance increment is eliminated (Figure 3.11A, box 3ii \& 5ii).

\section{A concatenated GlyRa2-mediated Circuit Enhances OFF RGC Surround Inhibition}

A concatenated inhibitory circuit (Figure 3.11B) also can explain GlyRa2mediated enhancement of RF surround suppression in WT OFF RGCs during the stimulation. This circuit requires three ACs that use serial and feedforward 
inhibition. Either a GABAergic or glycinergic AC (Figure 3.11B, AC2) expresses GlyRa2 to receive glycinergic input from a narrow field glycinergic AC (Figure 3.11B, AC1) and forms a serial inhibitory circuit in the Off sublayer, which modulates another GABAergic AC (Figure 3.11B, AC3) that uses feedforward inhibition to generate RF surround within the OFF RGC (Figure 3.11B). There is precedence in the literature for serial circuits and their disinhibitory mechanism (Chen et al., 2011; Eggers et al., 2007; Eggers and Lukasiewicz, 2010; Russell and Werblin, 2010; Zhang et al., 1997). Stimulus onset (luminance decrement within the RF surround of OFF RGCs) depolarizes the presynaptic OFF cone BCs (Figure 3.11B, box 1) and drives the serial AC-to-AC inhibition mediated by GlyRa2 to reduce/eliminate the inhibitory inputs in the downstream GABAergic OFF AC (Figure 3.11B, box2i \&3i) to increase its excitatory response. The disinhibited GABAergic OFF AC input drives a feedforward inhibition either directly onto the OFF RGC or indirectly onto its presynaptic OFF BC to generate the surround suppression (Figure 3.11B, box 4i). As a result, the surround suppression is enhanced by the serial circuit disinhibition (Figure 3.11B, box 5i). In the absence of GlyRa2, the serial circuit is disrupted (Figure 3.11B, box 2ii) and the feedforward inhibition generating the surround lost the enhancement by disinhibitory modulation (Figure 3.11B, box 3ii) and results in weaker surround (Figure 3.11B, box 4ii \& 5ii). 
A. Crossover-feedforward inhibition in the Off pathway enhances surround suppression and post-stimulus excitation

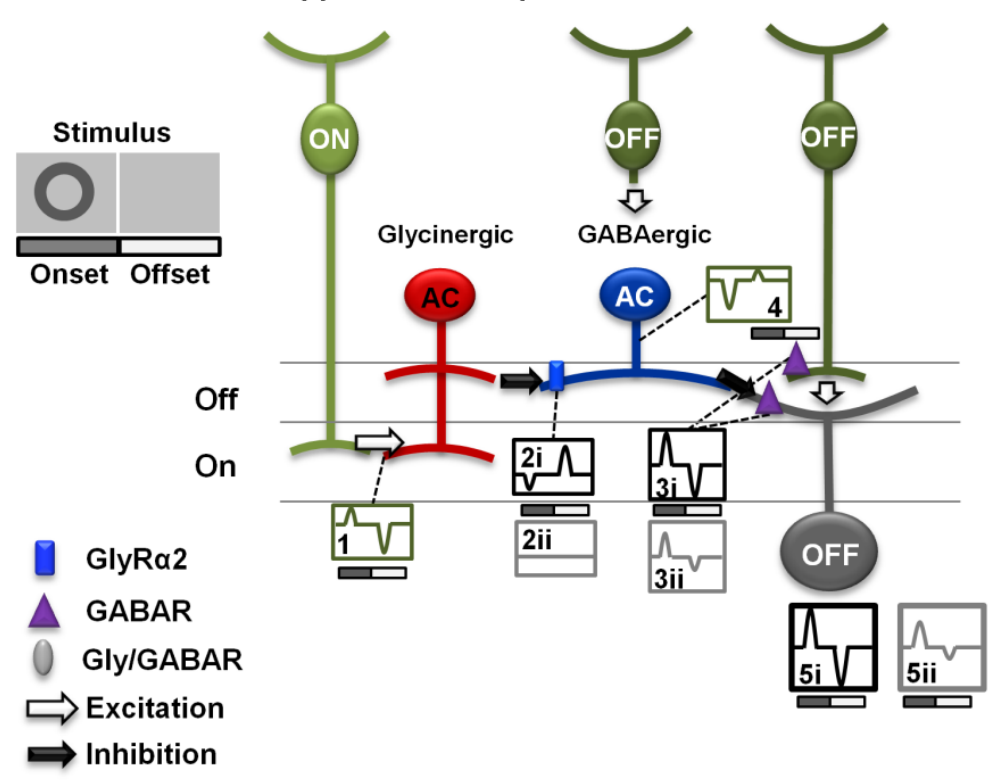

B. Concatenated inhibition in the Off pathway enhances surround suppression
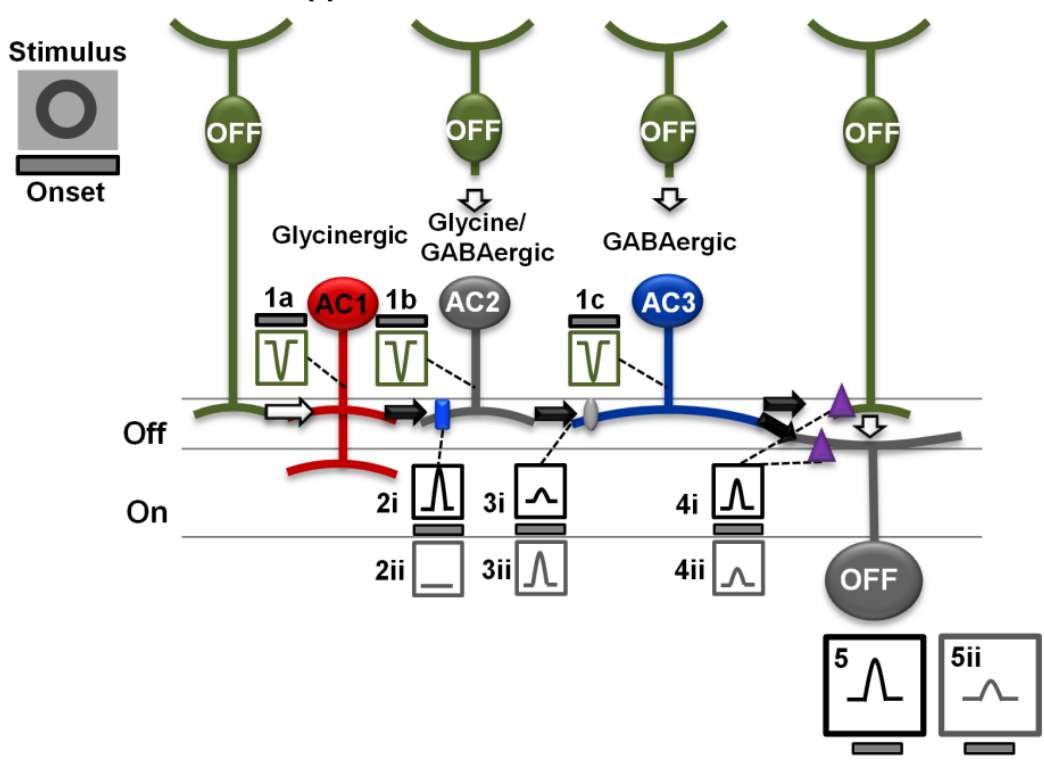

Figure 3.11. Schematic diagrams of the proposed roles of GlyRa2 in the WT Off mouse retinal pathways. Each diagram illustrates a basic retina circuit that is consistent with the results presented. The diagrams in the upper left corner illustrate the stimulus. Within each circuit the numbered boxes represent the 
current evoked at stimulus onset. N.B., currents are represented with transient time course for simplicity. The currents in the boxes do not reflect the temporal properties of the currents, as described in the results. (A) GlyRa2 mediates inhibition within OFF RGC receptive field surround using crossover-feedforward inhibitory circuit. GlyRa2 is expressed on a GABAergic AC (blue) to receive Onto-Off pathway crossover inhibition from a bi-stratified glycinergic AC (red). The GABAergic AC is responsible for generating the suppressive surround of OFF RGC at stimulus onset. At stimulus onset, the ON BC (light green) gets hyperpolarized (Box 1) and terminates the crossover inhibition onto the GABAergic AC (Box 2i), while the OFF BC (dark green) gets depolarized (Box 4) and activates the GABAergic AC. The On-to-Off glycinergic disinhibition combines with Off direct inhibition within the GABAergic $A C$ and enhances the its activity, which in turn increases the surround suppression of the OFF RGC (Box 3i \& 5i). At stimulus offset, the ON BC gets depolarized (Box 1) and drives the glycinergic $A C$ to inhibit the GABAergic $A C$ (Box 2i), while the OFF BC gets hyperpolarized and remove the excitatory drive of the GABAergic AC (Box 4). The glycinergic crossover inhibition combines with the termination of excitation within the GABAergic $A C$ and results in further decrease in the GABA release onto the OFF RGCs (Box 5i), which facilitates the offset rebound excitation. In the absence of GlyRa2, the GABAergic AC no longer receives the crossover inhibition (Box 2ii) and results in lower responses at both stimulus onset and offset (Box 3ii \& 5ii). (B) GlyRa2 mediate inhibition within RGC receptive field surround using concatenated inhibitory circuit. Three amacrine cells (ACs) form a 
train by making inhibitory synapses within the Off sublayer. The GlyRa2 (blue rectangle) is expressed on the dendrites of a glycinergic or GABAergic OFF AC (grey $A C$ in the middle) to receive inhibition from a glycinergic $A C$ (red) and mediate a serial inhibition onto the GABAergic wide field OFF AC (blue), which provides direct lateral inhibition onto the ON RGC. At stimulus onset (luminance increment), the ACs are driven by OFF cone bipolar cells (BCs), which produce inward currents (Box 1a-c). The glycinergic AC (red), via GlyRa2, produces an outward current in the middle OFF AC (Box 2i). The summation of outward and inward currents in the middle OFF AC results in a reduced outward current in the GABAergic OFF AC (Box 3i), which is summed with the inward current produced via input from its presynaptic OFF BC (Box 1c). The GABAergic OFF AC, using GABA receptors (GABARs, purple triangle), produces an outward current in the ON RGC and its presynaptic OFF BC (Box 4i \& 5i). In the absence of GlyRa2, the serial inhibition is disrupted (Box 2ii) and the GABAergic AC receives larger inhibition at luminance increment (Box 3ii). As a result, the OFF RGC received reduced GABAergic lateral inhibition within its RF surround (Box 4ii \& 5ii).

\section{GlyRa2-mediated Inhibition Modulate Both RF Center and Surround in WT OFF RGCs via Different Circuits}

Center-surround antagonism is the basic RF design of most RGCs and their interaction is essential for visual processing of contrast, spatial acuity and 
temporal selectivity (Cook and McReynolds, 1998; Enroth-Cugell and Robson, 1966; Greenberg et al., 2011; Kuffler, 1953; Rodieck and Stone, 1965). In the absence of GlyRa2, both the RF center and surround responses of OFF RGCs are reduced while maintaining their center:surround balance (Figure 3.10C). This suggests that GlyRa2-mediated inhibition modulates both the portion of the RF where the center and surround are contiguous, as well as in the RF surround that extends outside of the RF center. While this could represent a single GlyRa2 mediated mechanism, there is more evidence that supports the idea that there are different GlyRa2-mediated circuits that separately modulate the RF center and surround. First, our sample of OFF RGCs are most likely A-type RGCs as their large axons are most readily sampled by our electrodes in the optic nerve (Boycott and Wässle, 1974; Nobles et al., 2012). A-type OFF RGCs use crossover inhibition from the On pathway as the primary input to the RF center (Murphy at Rieke et al., 2006; Manookin et al., 2008; van Wyk et al., 2009; Zhang et al., 2014), and use feedforward inhibition from the Off pathway as the primary input to the RF surround (Cook and McReynolds, 1998; Flores-Herr et al., 2001; Roska et al., 2000). Therefore, the RF center and surround in OFF RGCs appear to be modulated by two distinct circuits driven by the On and Off pathways separately. Moreover, spatially the RF center and surround require ACs with different dendritic field sizes to perform this modulation, e.g., narrow field ACs modulate the RF center and wide field ACs the RF surround. Therefore, my results are most consistent with the interpretation that the RF center and surround of OFF RGCs are differentially modulated by GlyRa2-mediated circuits. 


\section{CHAPTER IV}

\section{ADENO-ASSOCIATED VIRUS-RNAI OF GLYRa1 AND CHARACTERIZATION OF ITS SYNAPSE-SPECIFIC INHIBITION IN OFF ALPHA TRANSIENT RETINAL GANGLION CELLS}

\section{Introduction}

In the mammalian retina, ACs form complex synaptic networks in a series of independent microcircuits (Anderson et al., 2011; Azeredo da Silveira and Roska, 2011; Helmstaedter et al., 2013; Marc et al., 2013). To regulate inner retinal excitatory signaling and shape the spiking output of retinal RGCs, these circuits utilize several mechanisms including feedforward, feedback, crossover and serial inhibition (reviewed in Zhang and McCall, 2012). As one of the main postsynaptic targets of ACs, GlyRs are critical components of inhibitory circuits and shape RGC spontaneous and visually evoked responses, e.g., temporal tuning or gain control (Chen et al., 2010, 2011; Manookin et al., 2008; Münch et el., 2009; Murphy and Rieke, 2006; Nobles et al., 2012; Pang et al., 2003; Roska et al., 2006; Russell and Werblin, 2010; van Wyk et al., 2009).

Four glycinergic a subunit subunits have been identified in mouse retina (Wässle et al., 2009; Dutertre et al., 2012) and each GlyR $\alpha$ subunit exhibits unique current decay kinetics and distinct cell class localization (Heinz et al., 
2007; Ivanova et al., 2006; Majumdar et al., 2007, 2009; Weiss et al., 2008). GlyRa1, the subunit with the fastest decay time, is expressed in OFF cone and rod bipolar cells (Eggers and Lukasiewicz, 2010; Ivanova et al., 2006) as well as in ON and OFF A-type RGCs (Majumdar et al., 2007). The other GlyR a subunits are localized on other retinal cell classes (reviewed in Zhang and McCall, 2012). The diversity of GlyRs, their different kinetics and retinal localization suggest that a subunit-specific circuits regulate visual signaling of either diverse properties within distinct circuits or similar properties across circuits. Supporting the idea of a role in distinct circuits, we showed that GlyRa2 and GlyRa3 selectively mediate inhibition in the retinal On and Off pathways, respectively (Nobles et al., 2012).

On-to-Off pathway glycinergic crossover inhibition onto presynaptic OFF cone BCs (Eggers and Lukasiewicz, 2010; Ivanova et al., 2006), as well as onto the RGCs, themselves (Majumdar et al., 2007; Manookin et al., 2008; Murphy and Rieke, 2006; Pang et al., 2003; van Wyk et al., 2009; Werblin, 2010) shapes the visual responses of OFF RGCs. These results and its retinal expression pattern make GlyRa1 the most likely subunit that modulates spontaneous and visually evoked activity of mature OFF a RGCs through crossover inhibition.

The lack of subunit specific antagonists and cell class specific/conditional GlyRa1 knockout mice preclude a direct test of this hypothesis. To address this dilemma and define the role of direct GlyRa1 input in shaping mature OFF $\alpha$ RGC responses, I combined several novel approaches. Namely, I: (1) infected adult RGCs with retrogradely transported AAV; (2) generated short hairpin RNA (shRNA) against Glra1 to eliminate its expression in RGCs without changing its 
expression in the upstream circuit; (3) targeted PV5 RGCs (likely OFF $\alpha_{\text {Transient; }}$; Münch et al., 2009) in a reporter mouse line $\left(P V^{C r e}\right)$ with eight identified and fluorescently labeled RGC types (Farrow et al., 2013) and (4) recorded spontaneous and visually evoked responses using two photon microscopy.

Compared to wild type, RNAi eliminates almost all GlyRa1 expression on the dendrites of PV5 (PV5 $\left.{ }_{W T}\right)$ RGCs, as well as all glycinergic sIPSCs and elPSCs. This indicates that the majority of glycinergic input to PV5 RGCs is GlyRa1 subunit specific. Because only the direct GlyRa1 input is eliminated in RGCs and the upstream circuitry is intact, differences in responses between PV5 RGCs with and without GlyRa1 input can be used to define its role in shaping the PV5WT response. I show that the GlyRa1 subunit mediates a previously described On-to-Off crossover synaptic input to OFF $\alpha_{\text {Transient }} R G C s$. I also show that in PV5 RGCs, a direct GlyRa1 specific inhibition controls spontaneous activity, facilitates excitatory signaling to luminance decrements (OFF response) and suppresses excitatory signaling to luminance increments (ON response). This improves the signal to noise ratio and maintains the correlation between the receptive field OFF center response and the stratification of the PV5WT RGC dendrites in the OFF sublaminae of the inner plexiform layer. Together these direct inhibitory inputs enhance the fidelity of PV5 ${ }_{W T}$ RGCs. I also demonstrate a complex interaction between glycinergic and GABAergic inputs at the level of the PV5 ${ }_{W T}$ RGCs. Namely, a serial GABA $A_{A}$ ergic input modulates GlyRa1 crossover inhibition and a direct $\mathrm{GABA}_{A}$ ergic inhibition modulates the PV5 ${ }_{W T} \mathrm{RGC}$ response at both luminance increment and decrement. 


\section{Results}

\section{PV5 ${ }_{W T}$ RGCs Express GlyRa1 and Have GlyRa1-mediated Synaptic Inputs}

I first examined the glycinergic subunit specific expression and currents in PV5WT RGCs. Previous reports observed expression of GlyRa1 puncta and slPSCs in A2 OFF RGCs (Sun et al., 2002), which should be the equivalent of

the PV5 RGC type, and their absence in the mouse mutant Glra $1^{\text {spd-ot }}$ (Majumdar et al., 2007). Using two photon microscopy, I targeted YFP-positive RGCs with neurobiotin. I verified their identity as PV5 RGCs in retinas triple-stained for: neurobiotin (Figure 4.1), choline acetyltransferase (ChAT; Figure 4.1Aii) and GlyRa1 (Figure 4.1B, C \& D), using their large soma and dendritic field size as well as the stratification of their dendrites, slightly proximal to the Off ChAT band in the IPL (Figure 4.1Aii; See also Münch et al., 2009; Farrow et al., 2013). Every PV5 ${ }_{\text {wT }}$ RGCs $(\mathrm{n}=8)$ had significantly more coincident GlyRa1 positive puncta compared to random coincidence (Figure 4.1Di, $0.27 \pm 0.03$ vs Figure 4.1Dii, $0.05 \pm 0.08$ puncta $\mu \mathrm{m}^{-1}$ of dendrite; $\left.p<0.0001\right)$. The corrected GlyRa1 coincidence, $0.21 \pm 0.02$ puncta $\cdot \mu \mathrm{m}^{-1}$, is similar to previous estimates in A2 RGCs (Majumdar et al., 2009). This is consistent with the idea that PV5 ${ }_{W T}$ are A2 OFF RGCs. 

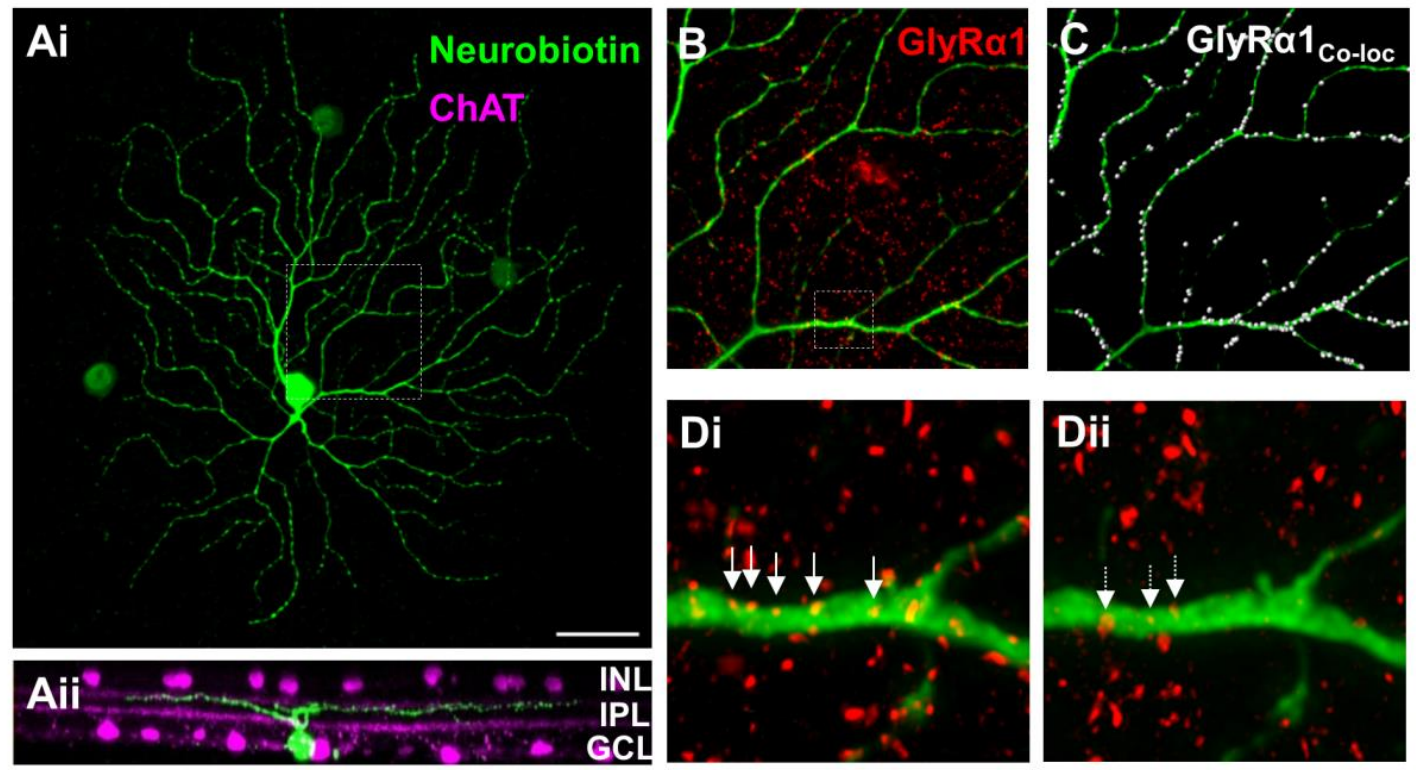

Figure 4.1. The GlyRa1 subunit is expressed on PV5 ${ }_{W T} R G C$ dendrites. (Ai)

Representative confocal image of a neurobiotin labeled PV5 RGC (green) in a retinal wholemount shows its characteristic A2 type morphology. (Aii) A rotated view of the same RGC shows its dendritic lamination pattern relative to the bands formed by the processes of the cholinergic amacrine cells (magenta) and that they are restricted to the Off sublaminae of the IPL. (B) Representative portion of the dendritic arbor of the same PV5 $5_{W T}$ RGC (see box; $70 \times 70 \mu \mathrm{m}$ area) and the punctate pattern of GlyRa1 subunit expression (red). (C) Co-localized GlyRa1 puncta (white dots) on the dendrite of the same RGC. (Di) A magnified and deconvolved image from B (see box). White arrows indicate a subset of representative co-localized GlyR 1 puncta on the PV5 dendrite. (Dii) Illustration of random association of GlyRa1 puncta that results from superposition of the channel containing the dendrite with a duplicated and $180^{\circ}$ rotated channel with the GlyRa1 puncta. White arrows indicate what are considered co-localized, 
albeit random, GlyRa1 puncta. The number of randomly co-localized puncta was used to correct GlyRa1 puncta coincidence rate. Abbreviations: INL, inner nuclear layer; IPL, inner plexiform layer; GCL, ganglion cell layer. Scale bar $=40$ $\mu \mathrm{m}$ in $\mathrm{A} ; 16 \mu \mathrm{m}$ for $\mathrm{B}, \mathrm{C} ; 2.5 \mu \mathrm{m}$ for $\mathrm{D}$.

In PV5 ${ }_{W T}$ RGCs in the presence of the GABA $A_{A}$ antagonist, picrotoxin (20 $\mu \mathrm{M}$; PTX), the majority (97\%) of glycinergic sIPSCs (Figure 4.2Ai and Aii) had decay times $\left(D_{37}\right)<5 \mathrm{~ms}$ and the distribution average was $2.7 \pm 0.03 \mathrm{~ms}$ (Figure 4.2B; 699 events; 12 PV5WT RGCs). This is identical to estimates of $\mathrm{T}$ for GlyRa1 sIPSCs in rat All cells recorded at physiological temperature (Gill et al., 2006), although faster than sIPSCs WT A2 OFF RGCs recorded at $25^{\circ} \mathrm{C}$ (Majumdar et al., 2007). My mean $D_{37}$ also is much faster than estimates for GlyRa2, $\alpha 3$ and a4 subunits (Wässle et al., 2009). In presence of both PTX and strychnine, all sIPSCs were eliminated (Figure 4.2Aiii) and the RGCs exhibited oscillatory current activity. My immunohistochemical and electrophysiological results indicate that that GlyRa1 inputs are the predominant glycinergic synaptic inputs of PV5 RGCs.

\section{Stimulus-evoked Response Profiles of PV5 ${ }_{W T} R G C s$}

Even though I used higher contrast stimuli, the receptive field (RF) center/ surround organization and stimulus-evoked spiking response properties of PV5 ${ }_{W T}$ RGCs are consistent with previous descriptions (Farrow et al., c.f. Figure 2; 
Münch et al., c.f. Figure 3). Namely, to a luminance decrement, PV5wT RGCs respond with an initial transient excitatory response followed by a sustained response slightly above spontaneous activity. To a luminance increment, there is a suppression in firing rate followed by a rebound excitation when spot diameters are small. The eEPSCs and eIPSCs that underlie the spiking response of PV5WT RGCs also are consistent with previous descriptions. A luminance decrement (onset of a $375 \mu \mathrm{m}$ dark spot, which matched the RF evokes only an eEPSC; whereas a luminance increment (offset of a dark spot) evokes only an eIPSC (Figure 4.2D). The magnitude of the eIPSC was unchanged when PTX eliminated $\mathrm{GABA}_{A}$ ergic inhibition (Figure 4.2D; $n=3 ; 205.84 \pm 66.01$ vs. 173.34 $\pm 58.77 \mathrm{nA} \cdot \mathrm{ms})$. In contrast, the elPSC was completely eliminated when strychnine $(10 \mu \mathrm{M})$ also was included. My morphological and electrophysiological results suggest that the previously described, glycinergic input to OFF $\alpha_{\text {Transient }}$ RGCs is GlyRa1 (Murphy and Rieke, 2006; Münch et al., 2009; van Wyk et al., 2009) and that this subunit input is a critical element of a crossover circuit, is maintained at high luminance and contrast levels. 
$\mathbf{A i}$

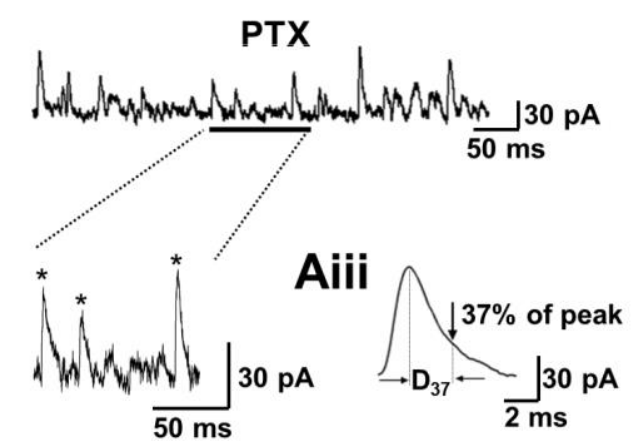

Aiv

PTX+Strychnine

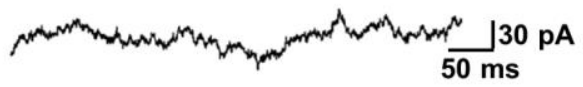

B

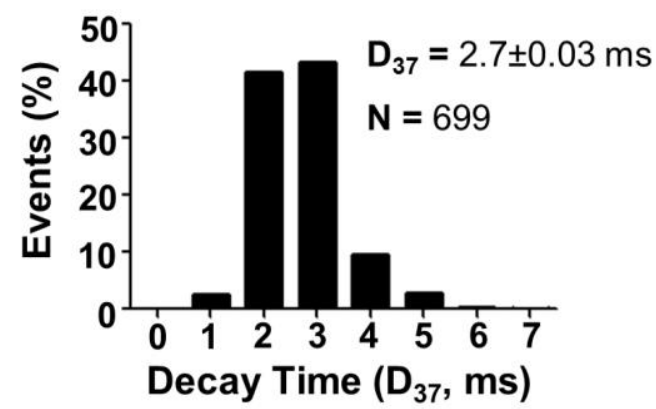

C $375 \mu \mathrm{m}$
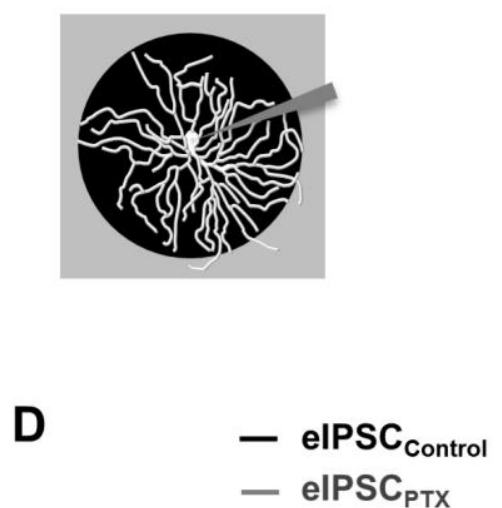

- elPSC $C_{\text {PTX }}$ -- eIPSC $_{\text {PTX }+ \text { Strychnine }}$

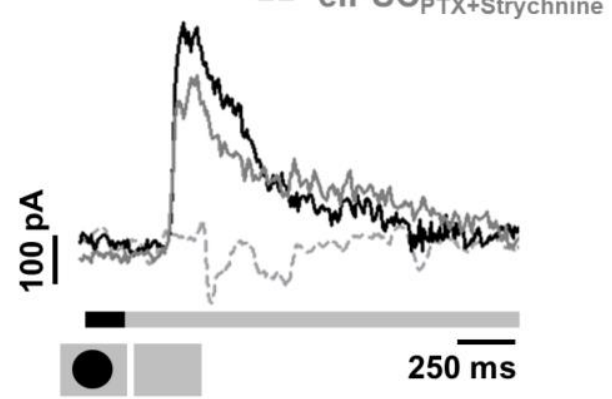

Figure 4.2. GlyRa1-meditated sIPSC and glycinergic elPSCs are present in PV5 $5_{W T}$ RGCs. (Ai) Glycinergic sIPSCs were recorded from PV5 ${ }_{W T}$ RGCs held at $0 \mathrm{mV}$ in the presence of picrotoxin (PTX, $20 \mu \mathrm{M}$ ). (Aii) Same trace as Ai with an expanded time scale. Stars indicate sIPSCs that met our criterion (see text) and were used in our analyses. (Aiii) Average waveform of PV5 ${ }_{W T}$ RGC sIPSCs that met the criterion (170 events) and an illustration of their average decay $\left(D_{37}\right)$. (Aiv) All sIPSCs were eliminated in presence of PTX $(20 \mu \mathrm{M})$ and strychnine (10 $\mu M)$. (B) The frequency of decay times $\left(D_{37}\right)$ in slPSCs of PV5 ${ }_{W T} R G C s(n=12$; 699 events). (C) A schematic representation of the visual stimulus, a dark spot $\left(6,000 \mathrm{R}^{*} / \mathrm{rod} / \mathrm{s}\right)$ centered on the PV5 ${ }_{\mathrm{WT}} \mathrm{RGC}$ soma, presented on a background $\left(24,000 \mathrm{R}^{\star} / \mathrm{rod} / \mathrm{s}\right)$. On average, a spot with outer diameter of $375 \mu \mathrm{m}$ matches the 
PV5 RGC dendritic arbor. (D) Averaged PV5WT RGC elPSC evoked by the offset of the stimulus shown in (C) in control solution. Picrotoxin (PTX, $20 \mu \mathrm{M})$ produced a small reduction in the eIPSC indicating a small GABAergic input. The eIPSC was completely eliminated when strychnine also was included (PTX 20 $\mu \mathrm{M}+$ strychnine $10 \mu \mathrm{M})$, indicating that the majority of synaptic inhibitory input is glycinergic.

\section{AAV-mediated RNAi Eliminates GlyRa1 Expression in PV5 GIra1-KD RGCs}

In examining the role of glycinergic input in the responses of RGCs, previous studies have used the competitive glycine antagonist, strychnine, which eliminates glycinergic signaling throughout the retinal circuit. I tested whether an RNAi approach could be used to selectively eliminate direct GlyRa1 subunit specific inhibition in RGCs while leaving the remaining upstream glycinergic inhibition intact. To this end, I injected AAVs expressing a Glra1-shRNA cassette into the dLGN to selectively target the AAV to RGCs. The viral vector plasmid (pAAV-Ef1a-NLStdTomato-H1; Figure 2.2) also expressed the tdTomato fluorescent protein with a nuclear localization signal, which I used to determine the timing and extent of RGC viral infection across the retina. I used RT-PCR and the cDNA isolated from HEK293 cells co-transfected with a plasmid expressing GlyRa1 along with either a AAV scrambled shRNA construct or one of three AAV 
Glra1-shRNA (a 29-mer and two 21-mer) constructs and evaluated the change in Glra1 transcription compared to a blank control vector (tdTomato only).
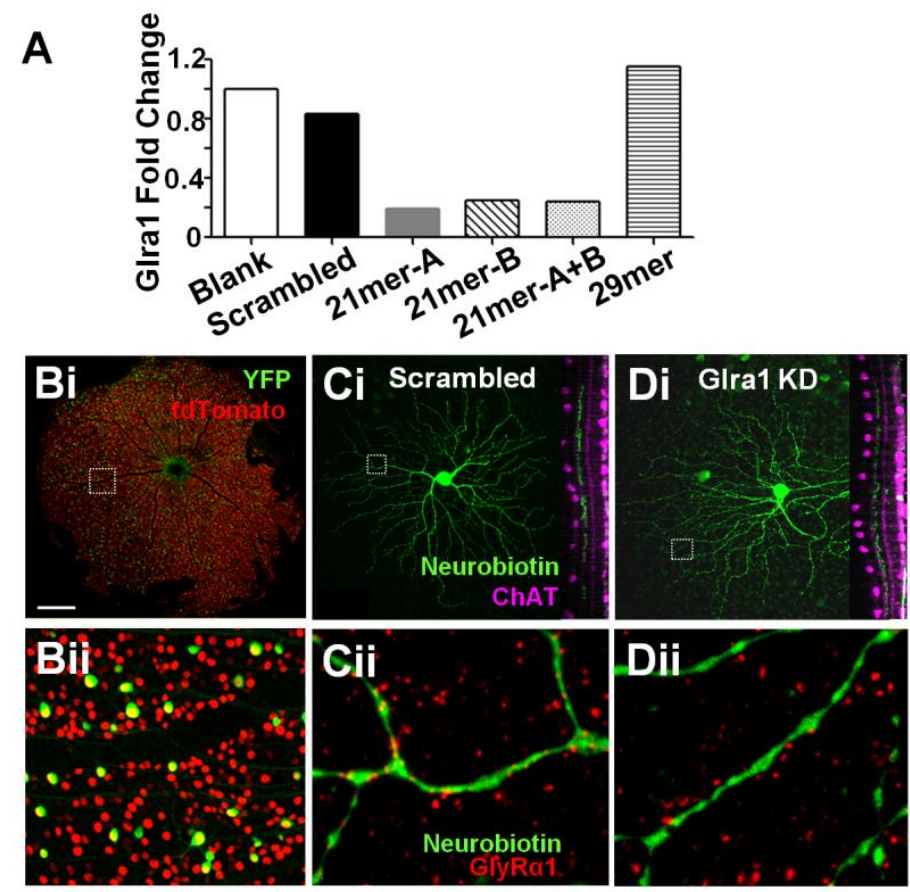

E

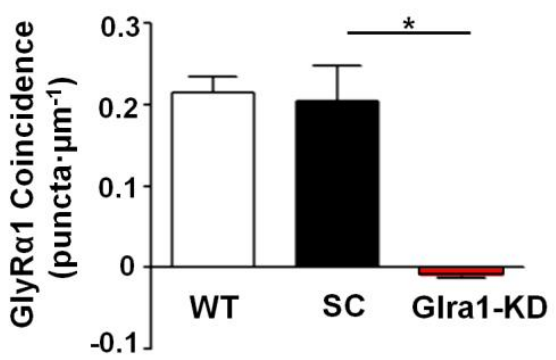

Figure 4.3. AAV-mediated RNAi significantly knocks down GlyRa1 expression on PV5 RGC dendrites. (A) RT-PCR results show that AAV-Glra1shRNA 21mer-A produced the largest fold reduction in Glra1 mRNA in transfected HEK293 cells compared to all other constructs. (Bi) Fluorescence confocal image of the contralateral retina of a $P v a l b^{C r e} \times$ Thy $1^{\text {Stp-EYFP }}$ mouse, 4 weeks after injection of AAV-scrambled shRNA into the dLGN. (Bii) Higher 
power image of the boxed area in $\mathrm{Bi}$, showing YFP positive PV RGCs (green), AAV infected RGCs with tdTomato positive nuclei (red) and double labeled PV infected RGCs (green with yellow nuclei). (C, D) Representative confocal images of neurobiotin filled PV5 RGCs infected with (Di) AAV-Scrambled-shRNA (SC) or (Di) AAV-Glra1-shRNA (Glra1-KD). (Cii \& Dii) Higher power images of the boxed areas in $\mathrm{Ci}$, Di showing the distribution of GlyRa1 expression (red puncta). (E) Dendrites of PV5 RGCs infected with AAV-GIra1-shRNA ( $n=7$ cells; $N=14$ dendritic fields) have significantly fewer coincident puncta than those infected with AAV-Scrambled-shRNA ( $n=4 ; N=8)$, whose expression is similar to PV5WT RGCs $(n=8 ; N=16)$. Scale bars $=600 \mu m(B i) ; 50 \mu m(B i i) ; 45 \mu m(C i, D i) ; 3 \mu m(C i i$, Dii).

One AAV construct, 21-mer-A Glra1-shRNA showed the most (81\%) down-regulation of Glra1 mRNA levels (Figure 4.3A), whereas the scrambled shRNA had no effect on Glra1 mRNA levels. All subsequent experiments in vivo used 21-mer-A Glra1-shRNA AAV. The sequences of the scrambled and Glra1 shRNAs are:

Scrambled shRNA: 5'- GTCGAAACCCGCAATAATAAT

Glra1 shRNA: 5'- GCACTACAACACAGGTAAATT -3'

Four weeks after LGN injection, I observed tdTomato nuclear expression throughout, but confined to the RGC layer in the contralateral eye (Figure 4.3Bi). 
Almost all YFP expressing $P V^{c r e}$ RGCs (green with yellow nuclei) were infected (Figure 4.3Bii). The remaining experiments targeted double labeled PV5 RGCs infected with AAVs generating scrambled (PV5 $\left.5_{S C}\right)$ or Glra1-shRNA (PV5 $5_{\text {Glra1-KD }}$ ).

To evaluate the effect of knockdown of Glra1 expression, I quantified coincident GlyRa1 puncta on the dendrites of PV5 $5_{S C}$ or PV5 $5_{\text {Glra1-KD; }}$ Figures $4.3 \mathrm{C}$ \& D). When corrected for random coincidence, there were no coincident GlyRa1 immunoreactive puncta on the dendrites of $\mathrm{PV} 5_{\mathrm{Glra1}-\mathrm{KD}}(n=7) \mathrm{RGCs}(-0.009 \pm 0.003$ puncta $\left.\mu \mathrm{m}^{-1} ; p=0.02\right)$. The density of GlyRa1 immunoreactive puncta on the dendrites of PV5SC $(n=4)$ was the same as PV5 ${ }_{\mathrm{WT}}(n=8)$ RGCs (Figure 4.3E; $0.20 \pm 0.04$ and $0.21 \pm 0.02$ puncta $\left.\cdot \mu \mathrm{m}^{-1}\right)$. I conclude that AAV-RNAi of G/ra1 significantly down regulates GlyRa1 protein expression in PV5 $5_{\text {Glra1-KD }} R G C s$ and that the scrambled construct has no off target effects. For the remainder of the experiments, I used PV5sC RGCs as controls. Retrograde AAV-RNAi significantly reduces GlyRa1expression and the majority ( 95\%) of glycinergic synaptic inputs. Without this glycinergic input, a PTX sensitive $\mathrm{GABA}_{A}$ ergic synaptic input is seen that is not evident in PV5 $5_{W T}$ or PV5 $5_{S C} R G C s$. I speculate that synaptic inhibition to PV5 ${ }_{W T} R G C s$ is modulated by two $\mathrm{GABA}_{A}$ ergic inputs. One inhibits a glycinergic AC and modulates spontaneous glycine release onto PV5 RGCs (Figure 4.8A, blue pathway). The other (Figure 4.8B, blue) modulates spontaneous GABA release onto PV5 RGCs (Figure 4.8B). I speculate that in PV5 ${ }_{W T}$ and PV5 $5_{S C}$ RGCs, PTX blocks the first, serial GABA $A_{A}$ ergic input and increases spontaneous glycine release. This offsets a decrease in sIPSC due to the PTX block of the direct spontaneous GABA release. The serial $\mathrm{GABA}_{A}$ ergic 
pathway is eliminated in $\mathrm{PV} 5_{\mathrm{Glra1}-\mathrm{KD}} \mathrm{RGCs}$, the direct $\mathrm{GABA}_{\mathrm{A}}$ ergic synaptic input can be observed as a consequence of its sensitivity/elimination by PTX.

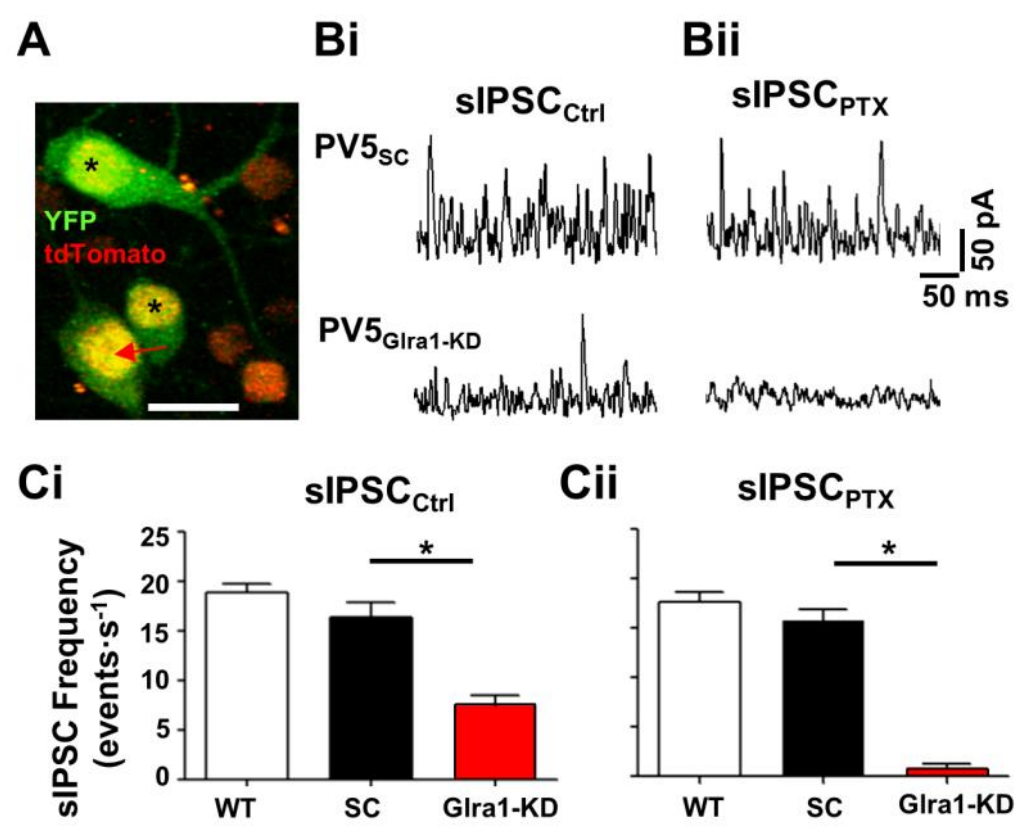

Di

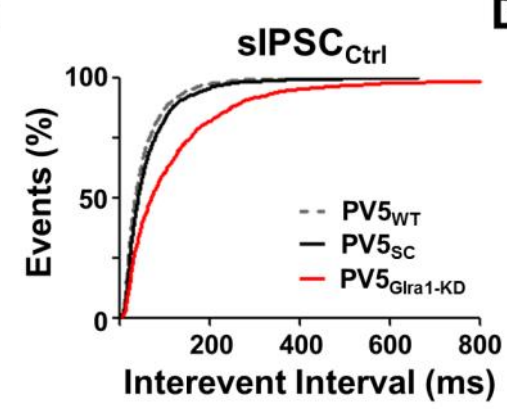

Dii

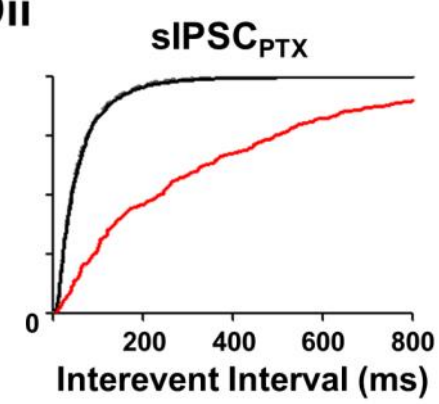

Figure 4.4. Knockdown of Glra1 eliminates GlyRa1 sIPSCs in PV5 RGCs. (A)

A representative two photon Z-stack image of a PV $5_{\text {Glra1-KD }} R G C$ infected by AAV-Glra1-shRNA (red arrow) that expresses YFP (green) in its cytoplasm and both YFP and tdTomato in its nucleus (yellow). Other $P V^{\text {cre }}$ RGCs types also are double labeled (*). RGCs labeled with GFP only represent infected, non $P V^{\text {cre }}$ 
RGC types (red). Scale bar $=40 \mu \mathrm{m}$. (Bi) Representative sIPSCs in PV5 5 and PV5 Gira1-KD RGCs. (Bii) Representative sIPSCs from the same RGCs in the presence of PTX $(20 \mu \mathrm{M})$. (Ci) In control solution, the average frequency of sIPSC in PV5 $5_{\text {Gira1-KD }} R G C s(n=7)$ is significantly lower compared to $P V 5_{W T}(n=7)$ and $\mathrm{PV}_{\text {SC }}(\mathrm{n}=5)$ RGCs, which are similar. (Cii) In the presence of PTX, the average sIPSC frequency in $\mathrm{PV} 5_{\text {Glra1-KD }}$ RGCs $(\mathrm{n}=7)$ also is significantly lower compared to $P V 5_{W T}(n=7)$ and $P V 5_{S C}(n=5) R G C s$, which are similar. Within PV5 $5_{\text {Glra1-KD }}$ RGCs, the sIPSC frequency is significantly reduced in control vs PTX. (D) Interevent intervals in $\mathrm{PV} 5_{\text {Gira1-KD }} R G C s$ sIPSC are longer than PV5 $5_{S C} R G C s$ in control (i) or in the presence of PTX (ii). Within PV $5_{\text {Glra1-KD }} R G C s$, the interevent intervals are significantly longer in PTX vs control.

\section{AAV-mediated RNAi Eliminates Stimulus Evoked GlyRa1 Currents in PV5 $5_{\text {Glra1-KD }} R G C s$}

A luminance increment depolarizes ON CBCs which initiates excitatory signaling in the On pathway. This stimulus culminates as a glycinergic inhibitory input onto PV5 ${ }_{W T}$ RGCs (dark spot offset; see Figure 4.2D). The elPSC total charge transfer evoked by this stimulus was similar across PV5 $5_{\mathrm{WT}}, \mathrm{PV} 5_{\mathrm{Sc}}$ and PV5 $5_{\text {Gra1-KD }}$ RGCs (Figure 4.5 A \& Bi; $n=3,5$ and 7; 205.83 $\pm 66.01,153.33 \pm 13.54$ and $136.62 \pm 13.34 \mathrm{nA} \cdot \mathrm{ms}$, respectively). However, an initial transient peak, found in the elPSC of both $P V_{W T}$ and PV5 $5_{S C} R G C s$, was absent in PV $5_{G \mid r a 1-K D}$ elPSCs (Figure 4.5Ai vs Aii, respectively). This flattened their eIPSC and significantly 
lengthened time to peak ( $157.6 \pm 30.4$ vs. $236.4 \pm 5.3 \mathrm{~ms} ; p=0.02)$. In the same cells, the presence of PTX did not alter the elPSC (elPSC $C_{\mathrm{PTX}}$ ) of PV5sC RGCs (Figures 4.5Ai, Aii \& C; $114.43 \pm 10.43 \mathrm{nA} \cdot \mathrm{ms}$ ). In contrast, elPSC $\mathrm{PTX}_{\text {in }} \mathrm{PV}_{5_{\mathrm{Gl}} \text { ra1-KD }}$ RGCs was significantly reduced the (Figure 4.5Aii; $30.49 \pm 6.31 \mathrm{nA} \cdot \mathrm{ms} ; p=0.001$ ). The elPSC $C_{\mathrm{PTX}}$ in $\mathrm{PV} 5_{\mathrm{Gira1}-\mathrm{KD}} \mathrm{RGCs}$ were glycinergic as they were eliminated by strychnine (Figure 4.5Aii) and likely result from the few GlyRa1 receptors that are expressed in PV5 ${ }_{\text {Glra1-KD }}$ RGCs. Further, their charge transfer was significantly smaller ( 27\%) than the glycinergic eIPSC in PV5sc RGCs (Figure 4.5A \& C; $p<0.0001)$. All of these changes in the elPSC are similar to those observed in sIPSCs, suggesting that the same two GABA $A_{A}$ ergic inputs modulate both synaptic and stimulus evoked responses (see Figure 4.8).

The comparisons of sIPSCs and elPSCs across PV5 ${ }_{W T}, P V 5_{S c}$ and PV5 ${ }_{G I r a 1-K D} R G C s$ show that: (1) the majority of glycinergic input to PV5WT $R G C s$ is mediated by the GlyRa1 subunit, (2) this RNAi approach effectively eliminates the majority of the synaptic and evoked glycinergic currents, (3) viral infection has no non-specific effect on spontaneous or evoked currents and (4) reveals the presence of two $\mathrm{GABA}_{A}$ ergic mechanisms that modulate synaptic inhibition in PV5 RGCs. 
Ai

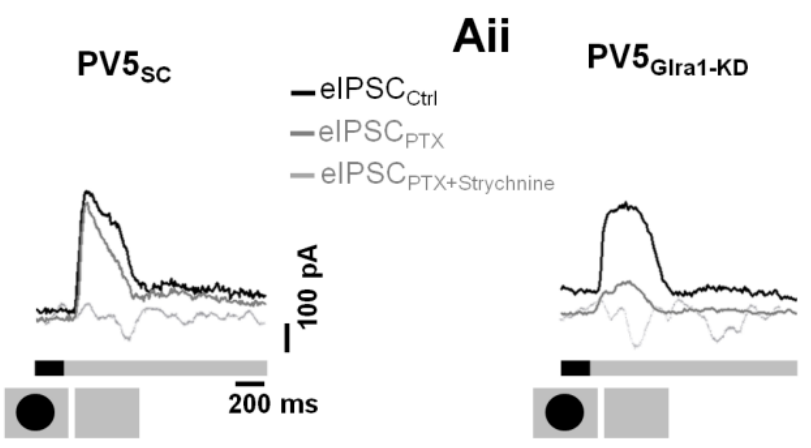

$\mathrm{Bi}$

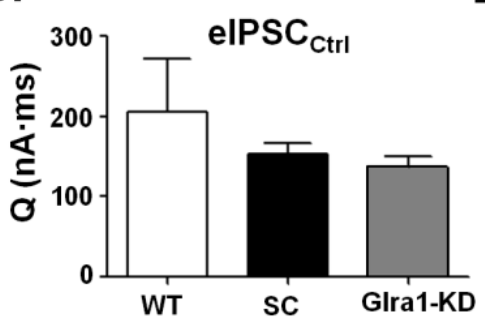

$\mathrm{Bii}$

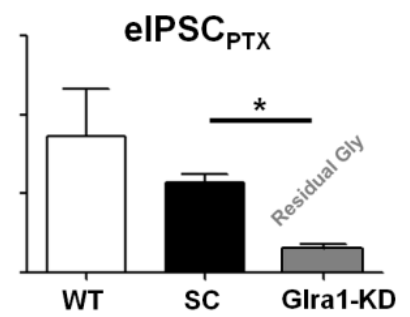

Figure 4.5. Knockdown of Glra1 eliminates GlyRa1 input to PV5 RGCs at luminance increments. (A) Representative elPSCs evoked by the offset of a dark spot (schematic below trace) from PV5 $5_{S C}(\mathbf{A i} ; n=5)$ and PV $5_{\text {Gira1-KD }}(\mathbf{A i i} ; n$ =7) RGCs in: control solution (black traces), in the presence of PTX (dark gray traces) and in the presence of both PTX and strychnine (light gray traces). In control $\mathbf{( B i )}$ the charge transfer does not differ across $\mathrm{PV} 5_{\mathrm{WT}}, \mathrm{PV} 5_{\mathrm{SC}}$ or $\mathrm{PV} 5_{\mathrm{Glra} 1-}$ KD groups, whereas the presence of PTX (Bii) significantly reduces the charge

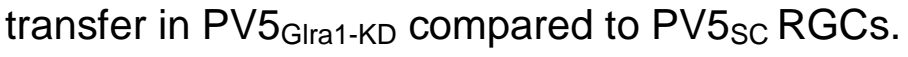




\section{PV5sc RGC Stimulus-evoked Responses}

Similar to WT, PV5 $5_{\mathrm{SC}} \mathrm{RGCs}$ respond to a luminance decrement (onset of a dark spot) with a transient excitatory peak ( $\leq 400 \mathrm{~ms}$ of stimulus onset) followed by maintained firing whose rate was above SA and matched to stimulus duration (2000 ms; Figure 4.6A,C \& D). A luminance increment (offset of a dark spot) evokes a short latency suppression of spiking that is followed by a small rebound excitatory response ( $\sim 500 \mathrm{~ms}$ from stimulus offset; Figure $4.6 \mathrm{~A} \& \mathrm{~B}$ ). The amplitude increases and then declines with increasing spot diameter and is not significantly above SA once the spot diameter exceeds the cell's RF center $(>500 \mu \mathrm{m})$. Given the difference in the stimulation conditions, my responses are

comparable to previously published PV5 ${ }_{W T}$ responses (Farrow et al., 2013) and do not vary with time after AAV infection.

\section{GlyRa1 Inhibitory Input Modulates PV5 ${ }_{W T}$ RGC Spontaneous Spiking}

\section{Activity and Post Suppression Rebound Responses to Luminance}

\section{Increments}

To define the roles of direct GlyRa1-mediated inhibition in PV5WT RGC visual function, I compared and defined the differences in the spontaneous and stimulus-evoked spiking responses of $\mathrm{PV} 5_{\mathrm{SC}}(n=12)$ and $\mathrm{PV} 5_{\mathrm{Glra1}-\mathrm{KD}}(n=13)$ RGCs. Consistent with a role for a tonic GlyRa1 inhibitory synaptic input to PV5 ${ }_{W T}$ RGCs, the absence of GlyRa1 current significantly increased the spontaneous spiking rate in PV5 ${ }_{\mathrm{Glra}} \mathrm{KD}_{\mathrm{KD}}$ compared to PV5 $\mathrm{SC}$ RGCs (Figure 4.6A; $13.95 \pm 2.60$ and $4.11 \pm 1.02$ spikes $\left.\cdot \mathrm{s}^{-1} ; p=0.003\right)$. 
This mismatch in SA precluded quantitative comparisons of spiking suppression to a luminance increment (Figure 4.6A). To correct for the difference in all other evoked spiking responses I subtracted the mean SA. The absence of GlyRa1 input did not alter RF center diameter in PV5 $5_{\mathrm{Glra} 1-\mathrm{KD}}$ compared to PV5 $5_{\mathrm{SC}}$ RGCs $(336.54 \pm 27.64$ vs $343.75 \pm 27.20 \mu \mathrm{m})$ and therefore I directly compared responses as a function of stimulus diameter.
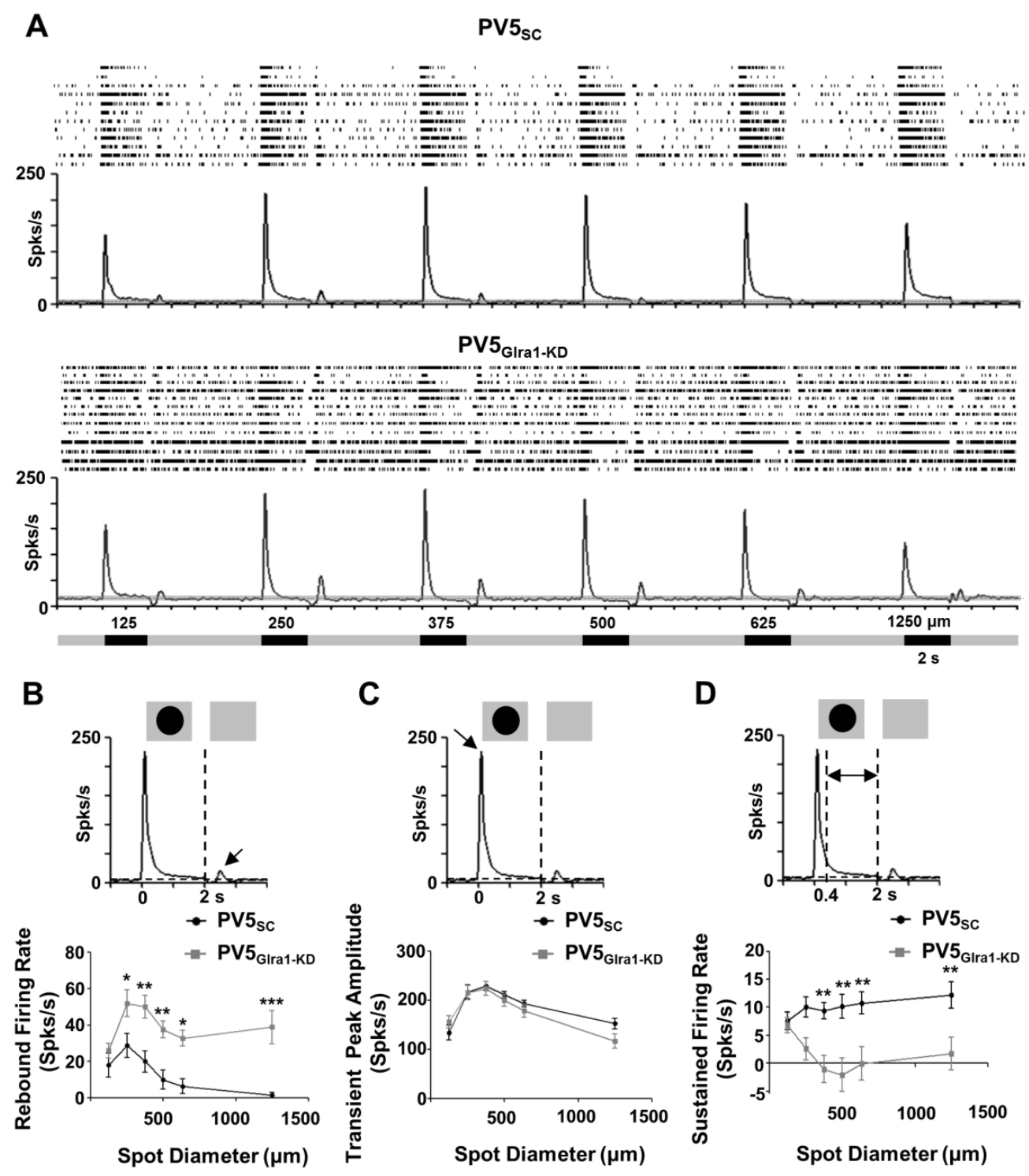
Figure 4.6. GlyRa1 input modulates PV5 RGC spontaneous activity and the excitatory rebound post stimulus suppression to a luminance increment. (A) Average PSTHs and individual raster plots (above) of the spiking responses of $\mathrm{PV}_{\mathrm{SC}}(\mathrm{n}=12)$ and PV5 $5_{\text {Glra1-KD }}$ RGCs $(\mathrm{n}=13)$ as a function of spot diameter. The mean SA is indicated by horizontal dashed lines on the PSTHs and grey shading indicates \pm 2 SE. Spot diameter and the onset/offset of the stimulus (described in Figure $3 \mathrm{~A}$ ) are represented in the diagram below the responses. (B) To a luminance increment, the rebound firing rate evoked at the offset of a dark spot, is significantly higher and a rebound response is retained across spot diameter in PV5 ${ }_{\text {Glra1-KD }}$ compared to PV5sc RGCs. (C) Transient peak amplitude does not differ between the two groups, although (D) sustained firing rates of $\mathrm{PV} 5_{\mathrm{Glra1}} \mathrm{KD}$ RGCs are significantly lower than PV5 $5_{S C}$ RGCs.

To parse the role of GlyRa1 in feedforward and crossover inhibitory mechanisms, I examined responses to luminance increments and decrements, respectively. In PV5sc RGCs, a luminance increase induced a suppression in the spiking response that was followed by a post suppression rebound response, as long as the spot diameter was $\leq$ the RGC RF center diameter. In PV5 $5_{\text {Glra1-KD }}$ the rebound response did not decline significantly with increasing spot diameter, although it was significantly larger than PV5sc RGCs at all but the smallest spot diameter (Figure 4.6A \& B; Two-way ANOVA, $p<0.0001$ ). The rebound response 
had a slow onset and had no directly associated eEPSC. Although a small inward current was recorded with similar timing (Figure 4.7A), it did not differ across $\mathrm{PV} 5_{\mathrm{WT}}, \mathrm{PV} 5_{\mathrm{SC}}$ and $\mathrm{PV} 5_{\mathrm{Glra1}-\mathrm{KD}} \mathrm{RGCs}$. This result along with the observation that an intrinsic rebound response can be induced in WT OFF RGCs by injecting negative current (Margolis and Detwiler, 2007), suggests that an absence of tonic GlyRa1 input causes PV $5_{\text {Gira1-KD }} R$ GCs resting membrane potential to be more depolarized. At the end of spike suppression, that results from a release from inhibition, spiking threshold is reached sooner in the more depolarized PV5 $5_{\text {Gira1-KD }} R G C s$ and results in an enhanced rebound response.
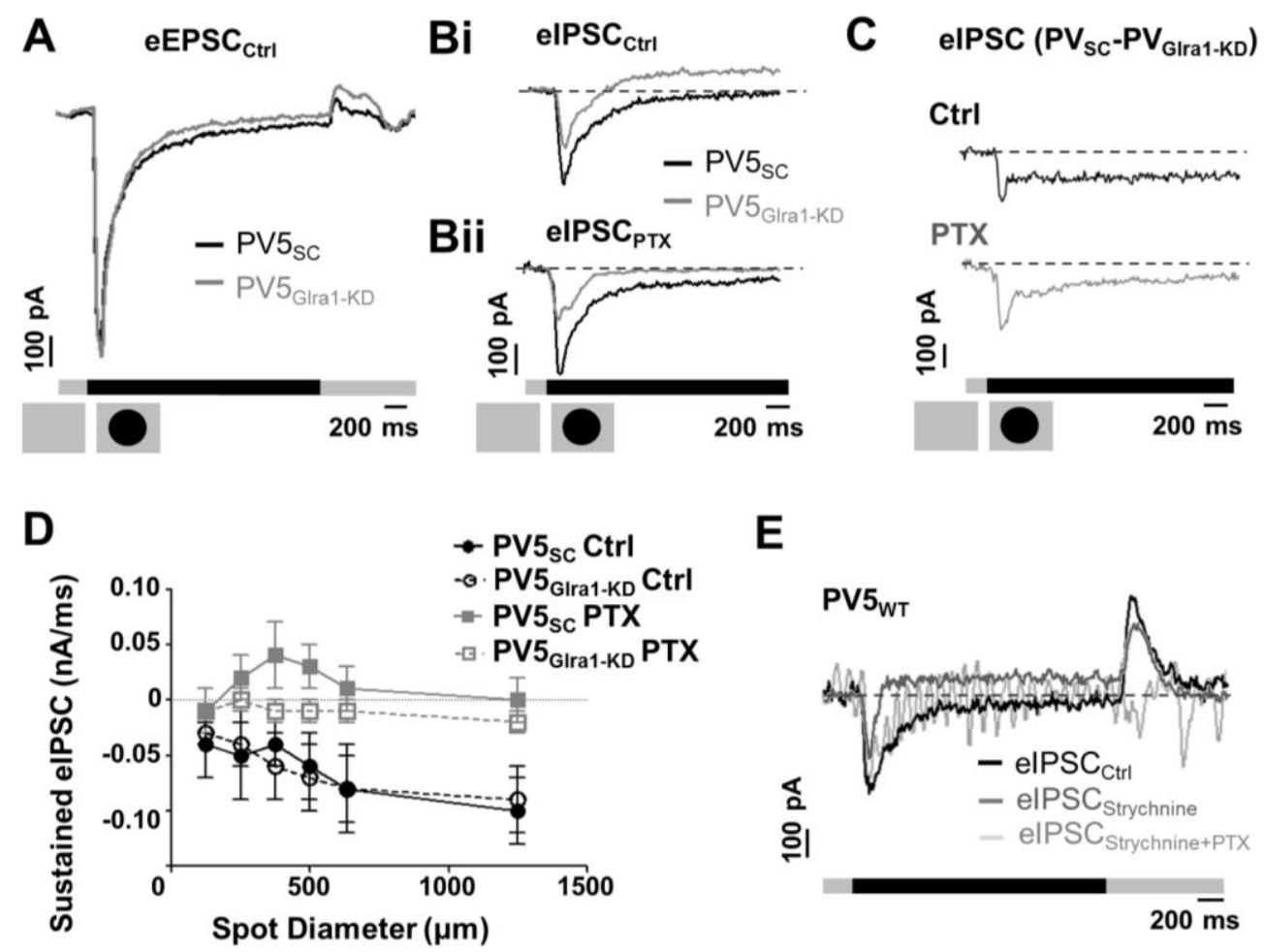

Figure 4.7. Stimulus evoked responses to luminance decrement is modulated by glycinergic and $\mathrm{GABA}_{A}$ ergic inputs. (A) To a luminance 
decrement (375 $\mu$ m diameter spot), the average eEPSC of PV5sc (black, $n=4$ ) and PV5 $5_{\text {Glra1-KD }} R G C s$ (grey, $n=5$ ) are similar. (B) To the same stimulus, the average eIPSC of PV $5_{G i r a 1-K D}(n=7)$ is significantly elevated compared to PV $5_{S C}$ RGCs ( $n=5)$ in control solution (black) and in the presence of PTX (grey). (C) The subtraction of the average elPSC of $\mathrm{PV} 5_{\mathrm{Glra} 1-\mathrm{KD}}$ from $\mathrm{PV} 5_{\mathrm{SC}}$ under control (black) show a prominent decrease in eIPSC at luminance decrement, suggesting a disinhibition. This decrease also is seen in the presence of PTX (grey). (D) The mean sustained elPSC of PV5 $5_{S C}$ (black circles) and PV $5_{\text {Gira1-KD }}$ RGCs (grey squares) in control solution (solid symbols) and in the presence of PTX (open symbols) as a function of spot diameter. The significantly elevated eIPSC level in PV5 $5_{\text {Gira1-KD }}$ RGCs in control and in the presence of PTX indicates the elimination of glycinergic disinhibition. The absence of glycinergic disinhibition unmasks a $\mathrm{GABA}_{A}$ ergic input in PV5 $5_{\text {Gra1-KD }}$ RGCs, which is blocked by PTX. (E) elPSC from a PV5WT RGC in the presence of strychnine (grey) exhibits a similar response profile to $\mathrm{PV} 5_{\mathrm{Gira1}-\mathrm{KD}} \mathrm{RGCs}$ in control solution that differs from control (black). 


\section{GlyRa1 Disinhibitory and GABA Inhibitory Inputs Use A Push-pull}

\section{Mechanism to Modulate PV5 wT RGC Responses to Luminance Decrements}

A luminance decrease depolarizes OFF CBCs and initiates excitatory signaling in the Off pathway that culminates with an excitatory response in PV5 ${ }_{W T}$ RGCs was similar (Figure 4.6C), but the sustained firing rate was significantly lower in PV5 ${ }_{\mathrm{Glra1} 1 \mathrm{KD}} \mathrm{RGCs}$ across spot diameter (Figures 4.6D; $p<0.0001$ ). This result appears inconsistent with the observation that no inhibitory current is evoked in PV5WT RGCs to this stimulus (Figure 4.7E; see also Münch et al., 2009). To address this discrepancy, I characterized and compared the stimulusevoked currents of PV5 5 and $\mathrm{PV} 5_{\mathrm{Glra1}-\mathrm{KD}}$ elPSCs to a luminance decrement.

The transient component of the responses of $\mathrm{PV} 5_{\mathrm{WT}}, \mathrm{PV} 5_{\mathrm{SC}}$ and $\mathrm{PV} 5_{\mathrm{Gira}}$ KD RGCs held at $0 \mathrm{mV}$, appears as an inward current (Figure 4.2C \& 4.7B; See also Münch et al., 2009). This reflects incomplete voltage clamp (e.g., space clamp) due to the large dendritic fields and gap junctional coupling in PV5 RGCs. Since the AAV-RNAi does not affect presynaptic inputs and the eEPSCs are similar between $\mathrm{PV} 5_{\mathrm{SC}}$ and PV5 ${ }_{\text {Glra1-KD }}$ RGCs (Figure 4.7A), I conclude that space clamp is similar between the two groups and the absence of GlyRa1 input does not affect the gap junctional coupling or the dendritic field electrotonic properties of PV5 RGCs. Therefore, differences between PV5SC and PV5 $5_{G i r a 1-K D}$ eIPSCs reflect the role of GlyRa1 input in their stimulus evoked responses.

A luminance decrement evoked a prominent sustained elPSC in PV5 $5_{\text {Gira1- }}$ ${ }_{\text {KD }}$ RGCs that was absent in PV5 ${ }_{\mathrm{SC}}$ RGCs (Figure 4.7Bi). Consistent with this, the 
mean sustained elPSC was significantly higher in $\mathrm{PV} 5_{\mathrm{Glra}}$-KD compared to $\mathrm{PV} 5_{\mathrm{SC}}$ (Figure 4.7C \& D; $n=7$ vs. 5$)$ in both control $(p<0.0001)$ and in the presence of PTX $(p<0.0001)$ and this difference was significant across spot diameters (Figure 4.7D). These results define a role for GlyRa1 subunit input in a previously described crossover glycinergic mechanism that facilitates excitation in OFF RGCs to a luminance decrement (Murphy and Rieke, 2006; Manookin et al., 2008). Moreover, the glycinergic modulation is spatially invariant and is not altered by recruitment of lateral inhibitory mechanisms with increasing spot diameter. Because the sustained elPSC eliminated by PTX in PV5 $5_{\text {Gira1-KD }}$ RGC was not evident in PV5 5 RGCs (Figure 4.7Bii \& D; $p=0.01$ ), I eliminated glycinergic input in PV5 ${ }_{W T}$ RGCs with $10 \mu \mathrm{M}$ strychnine and determined that a $\mathrm{GABA}_{A}$ ergic elPSC was present to a luminance decrement that could be reduced by PTX (Figure 4.7E).

My data show that to a luminance decrement, the responses of PV5WT RGCs are modulated by GlyRa1 using a mechanism that has been previously described as an On-to-Off crossover glycinergic disinhibition that enhances sustained spiking activity (Pang et al., 2003; Murphy and Rieke, 2006; Manookin et al., 2008; van Wyk et al., 2009). In addition, my data define a GABA ${ }_{A} e r g i c$ input that is driven through the Off pathway and reduces spiking activity in PV5 RGCs. This suggests that a combination of GABAergic and glycinergic inputs uses a "push/pull" mechanism to control PV5 ${ }_{W T}$ RGCs to a luminance decrement (Figure 4.8). 


\section{Discussion}

As an image processor, the retina dynamically encodes visual inputs using distinct RGC types where different inhibitory mechanisms modulate the excitatory signal and shape visual function. Much of our view of this signaling in the retina has relied on pharmacological manipulations, which alter not only the direct effects of the agonist/antagonist but also effects on these inputs in the upstream circuit. I show that viral RNAi can be used to selectively eliminate both expression and a subunit-specific current in an OFF RGC subtype, the PV5 RGC, while maintaining their upstream circuit intact. In addition, I show that this approach can be used to evaluate the role of a specific glycinergic subunit in visual function in identified RGCs. My results show that GlyRa1 input to PV5 RGCs is the predominant crossover glycinergic input described previously in OFF $\alpha_{\text {Transient }}$ RGCs (Manookin et al., 2008; Murphy and Rieke, 2006; Pang et al., 2003; van Wyk et al., 2009). In addition, the data show that both glycinergic and GABAergic inputs shape PV5 RGC spontaneous and visually-evoked responses and modulate their interaction differentially at luminance increment and decrement.

\section{AAV-mediated Infection of RGCs with shRNA Reduces GlyRa1 Expression and Currents}

AAV-RNAi has been adopted in a variety of biological systems to induce and/or eliminate gene expression (reviewed in Borel et al., 2014). In the retina, $A A V s$ have been used primarily in gene therapy approaches to treat retinal 
disease (reviewed in McClements and MacLaren, 2013; Sahel and Roska, 2013) and more recently in combination with pseudotyped rabies virus to trace presynaptic partners (Cruz-Martin et al., 2014) or target and characterize the visual response properties of identified RGC types (Farrow et al., 2013; Yonehara et al., 2013).

My study shows a novel use of retrogradely transported AAV-RNAi eliminates expression of a subunit-specific receptor in an identified RGC type. I use the approach to define the receptor's role in shaping the visually-evoked response properties of PV5 RGCs and the underlying excitatory and inhibitory currents. The advantages of this AAV-RNAi approach include: (1) its use in mature tissue to circumvent developmental complications and (2) its relatively rapid expression ( $\leq 4$ weeks) and (3) its long-term stability of expression (up to 10 weeks in my study) and (4) its selectivity to its target. Retrogradely transported AAV-RNAi is a particular benefit, when a circuit, like the retina, expresses a receptor subunit at multiple locations (e.g., glycinergic and $\mathrm{GABA}_{A}$ ergic inputs) and when subunit specific agonist/antagonists do not exist. My results show that AAV-RNAi can be used to selectively eliminate the majority of GlyRa1-mediated inputs to an identified RGC, the PV5. Finally, my results show that the approach dissects complex interactions between GABAergic and glycinergic inputs. Given that retrograde transport is similar throughout the central nervous system, this approach should be broadly applicable to both inhibitory and excitatory receptor mediated inputs. 


\section{Tonic GlyRa1 Input Modulates Spontaneous Activity and Rebound Excitation of PV5 $5_{W T} R G C s$.}

I used differences between $\mathrm{PV} 5_{\mathrm{Gl} \text { Ira1 }} \mathrm{KD}$ and $\mathrm{PV} 5_{\mathrm{SC}} \mathrm{RGC}$ responses to define GlyRa1 mediated inputs to PV5 ${ }_{W T}$ RGCs that are initiated in both the On and Off pathways. In general, the glycinergic mechanisms that modulate PV5 RGC spontaneous and stimulus-evoked responses act to enhance the fidelity of their spiking responses and the signal that they convey to the rest of the brain.

Under a steady background, a GlyRa1-mediates a tonic input to PV5 RGCs that reduces their spontaneous spiking activity. This is likely the same as the glycinergic current that has been previously observed to lower spontaneous activity and improve the signal to noise ratio in OFF RGCs (Margolis and Detwiler, 2007; Pang et al., 2003; Zaghloul et al., 2003). I conjecture that this control occurs via a GlyRa1 input that helps to set the resting membrane potential of the PV5 RGCs. In the absence of GlyRa1-mediated currents, I found $\mathrm{GABA}_{A}$ ergic synaptic inputs whose role is unknown.

A luminance increment depolarizes ON CBCs and culminates in a transient suppression of spiking activity followed by rebound excitation in PV5WT RGCs. The absence of GlyRa1 input enhances this rebound excitation. I observed a small inward current that is temporally related to this response, which could be generated by the excitatory input from BCs (Kastner and Baccus, 2013; Nikolaev et al., 2013) or could be related to the intrinsic rebound response that is evoked when negative current is applied and all synaptic input is blocked to OFF RGCs (Margolis and Detwiler, 2007). Alternatively, if GlyRa1 input contributes 
to the PV5 RGC resting membrane potential it should make the PV5 RGC more hyperpolarized and lower the probability that the release from inhibition will initiate an excitatory response to a luminance increase. Regardless of its exact role, the presence of GlyRa1 input maintains the correlation between PV5 RGC dendritic lamination in the Off IPL sublaminae and their RF OFF center response.

\section{Both Feedforward and Crossover Inhibitory Circuits Regulate PV5 $5_{W T}$ RGCs Light Responses at Luminance Decrement}

A luminance decrement depolarizes OFF CBCs (Figure 4.8A, box 4, inward current), whereas ON CBCs hyperpolarize (Figure 4.8A, box 1, outward current). This culminates in a large transient spiking response in PV5 ${ }_{W T}$ RGCs, which is followed by a much lower sustained spiking response matched in duration to the stimulus. The absence of GlyRa1 input does not alter the transient peak or their eEPSC, but results in a significantly lower sustained spiking rate compared to PV5 ${ }_{S C}$ and PV5 ${ }_{W T}$ RGCs. This suggests that the GlyRa1 inhibitory input is likely to be a previously described glycinergic On-to-Off crossover tonic input (Demb and Singer, 2012; Ke et al., 2014; Münch et al., 2009; Murphy and Rieke, 2008), facilitates excitation in PV5 RGCs (glycinergic disinhibition; Figure $4.8 \mathrm{~A}$, box $3 \mathrm{i} \& 5 \mathrm{i})$. If this is the case, this means that the All, defined as the presynaptic $A C$ in the crossover circuit operates at the high background and contrast levels used here. Alternatively, one of the other multistratifed ACs (Buldyrev et al., 2012; Menger et al., 1998) could be the presynaptic AC. I found that this stimulus evokes a direct $\mathrm{GABA}_{\mathrm{A}}$ ergic input (Figure 4.8B, box 4), which 
should suppress spiking activity and indicates that a push-pull mechanism mediated by $\mathrm{GABA}_{A}$ ergic and GlyRa1 inputs set the spiking output of PV5WT RGCs to a luminance decrement.

A. Serial GABA $A_{A}$ ergic inhibition modulates GlyRa1-mediated crossover synaptic input

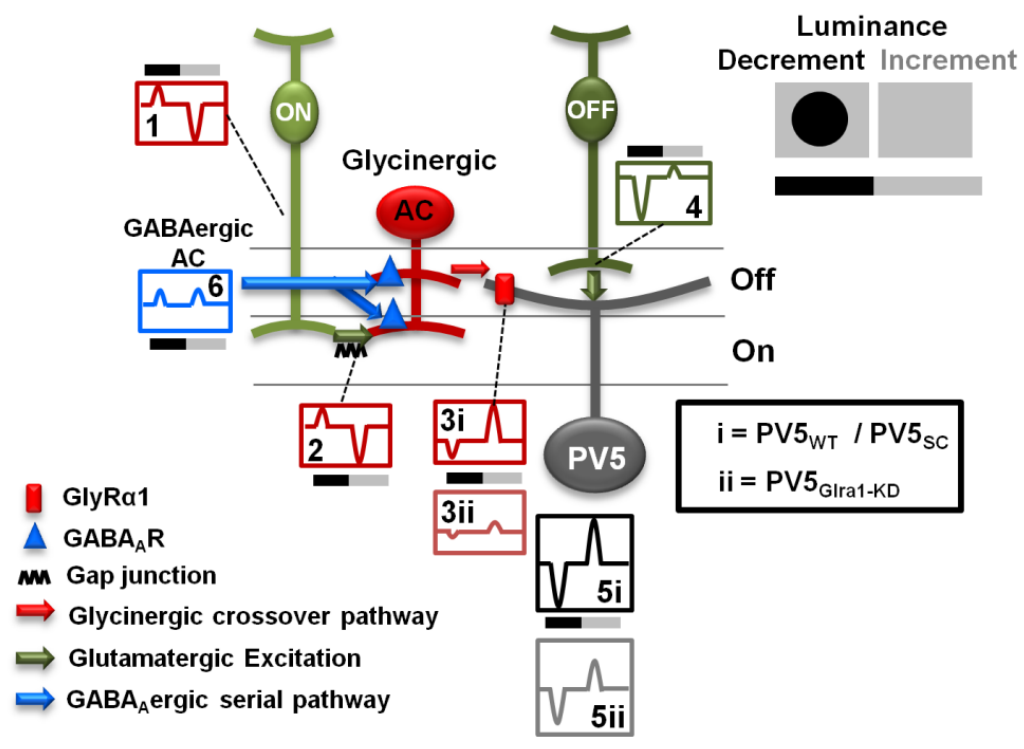

B. Parallel $G A B A_{A}$ ergic feedforward synaptic input

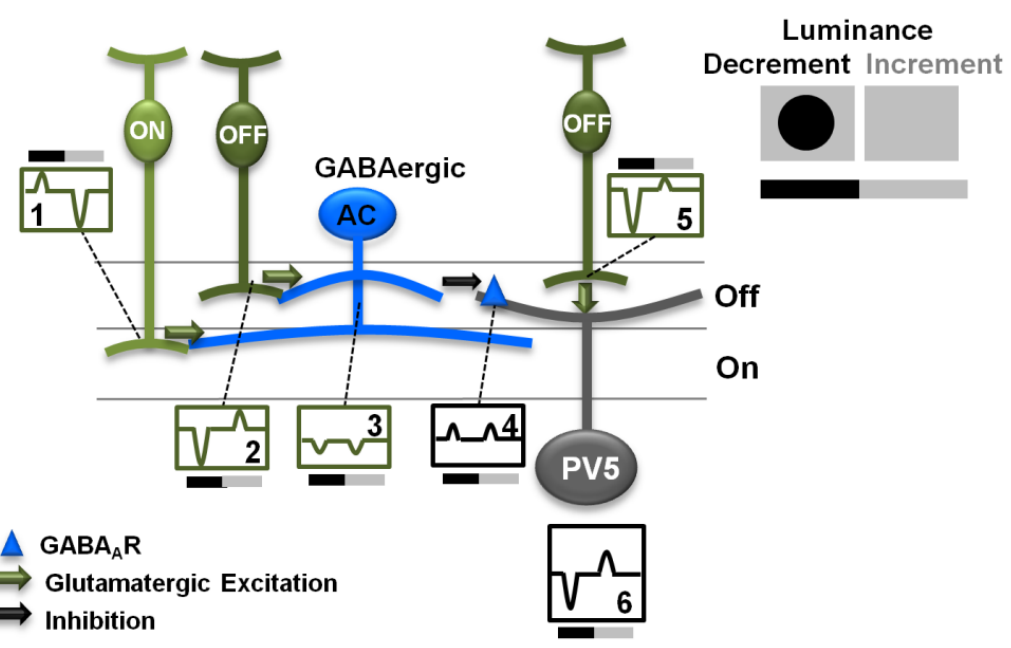

Figure 4.8. The light evoked responses of PV5 $5_{W T} R G C s$ are modulated by both GlyRa1 and GABA $A_{A}$ ergic inputs. Schematic diagrams of the circuits 
controlling light evoked responses of PV5 $5_{W T}$ RGCs. (Upper right). A diagram illustrating the stimulus, a dark spot presented on a light adapting background and centered on the RF center. Below the bars indicate the timing of spot onset (luminance decrement) and offset (luminance increment). NOTE: Current onset and offset are accurately depicted, but all currents are shown as transient. (A) A GlyRa1-mediated crossover inhibitory circuit modulates the responses of PV5 RGCs at luminance increment and decrement. PV5 $5_{W T}, P V 5_{S C}$ and $P V 5_{G i r a 1-K D}$ ON CBCs (Box 1) drive bistratified glycinergic ACs (Box 2). In PV5 ${ }_{W T}$ and PV5 $5 \mathrm{sc}$ this glycinergic input produces inhibition via their GlyRa1 postsynaptic receptors (rectangle on dendrite). A synaptic rectification occurs between the ON CBC and the bistratified glycinergic AC resulting in a small outward current at luminance decrement and a large inward current at luminance increment (Box 2). Through this receptor the glycinergic ACs evoke currents of inverted polarity in the PV5 RGC (Box 3i). The net result (Box 5i) is disinhibition at luminance decrement and inhibition at luminance increment. Knockdown of Glra1 eliminates this crossover inhibition and reduces excitation at luminance decrement and inhibition at luminance increment (Box 3ii, 5ii). The glycinergic $A C$ is regulated by serial $\mathrm{GABA}_{\mathrm{A}}$ ergic input (triangle) at luminance decrement and increment (Box 6). (B) A feedforward $\mathrm{GABA}_{A}$ ergic inhibition arises in both the ON and the OFF pathway and suppresses $\mathrm{PV} 5_{\mathrm{WT}}, \mathrm{PV} 5_{\mathrm{SC}}$ and $\mathrm{PV} 5_{\mathrm{Glra1}-\mathrm{KD}} \mathrm{RGC}$ responses at luminance increment and decrement, respectively (Box 4 \& 6). It is conducted by multistratified GABAergic ACs (Box 3). 


\section{Glycine and GABA Inputs Suppress PV5 RGCs Light Responses at}

\section{Luminance Increment}

The sustained suppression to a luminance increment (discussed above) is followed by a rebound excitatory response. Although a fast, transient GlyRa1 input is eliminated in PV5 $5_{\text {Gra1-KD }} R G C s$, the suppressive response remains. This suggests that spiking suppression is produced through an On to Off crossover pathway consisting of a transient GlyRa1 and a prolonged $\mathrm{GABA}_{A}$ ergic input. Unlike the push-pull combination of feedforward $\mathrm{GABA}_{A}$ ergic and crossover glycinergic inputs at luminance decrement, the $\mathrm{GABA}_{A} \mathrm{ergic}$ and glycinergic crossover inputs at luminance increment work together.

\section{A Serial GABA-glycine Circuit Masks the Direct GABA $A_{A}$ ergic Input in PV5 ${ }_{W T}$ RGCs}

In the retina, serial connections among amacrine cells (Anderson et al., 2011; Chen et al., 2011) are thought to fine tune downstream activity. Serial GABA-to-glycine or glycine-to-GABA inhibition has been observed in both RGCs and BCs (Eggers et al., 2007; Eggers and Lukasiewicz, 2010) and modulates RGC RF center activity (Russell and Werblin, 2010; Venkataramani and Taylor, 2010; Zhang et al., 1997). I interpret my results to indicate that PV5 (OFF

$\left.\alpha_{\text {Transient }}\right) R G C$ s receive a serial GABA-glycine inhibition (Figure $4.8 \mathrm{~A}$, box 6 ) and a direct GABAergic inhibition (Figure $8 B$, box 4 ) both mediated by $\mathrm{GABA}_{\mathrm{A}} \mathrm{R}$. In the presence of the $G A B A_{A} R$ antagonist, picrotoxin, both serial and direct GABAergic inhibition are blocked, producing offsetting conductance changes. 
This makes it virtually impossible to separate the two mechanisms. The selective elimination of the direct glycinergic input in $\mathrm{PV} 5_{\mathrm{Glra} 1-\mathrm{KD}} \mathrm{RGCs}$ also eliminates the GABA-glycine serial modulation, and unmasked the direct $\mathrm{GABA}_{A}$ ergic input. Providing support for this hypothesis, I also could observe a GABA $A_{A}$ ergic component in PV5 ${ }_{W T}$ RGCs when glycinergic input was eliminated in the presence of stychnine (Figure 4.7E). While this scenario represents a complex interaction, there is other support for my hypothesis. For example, the likely amacrine cell that provides glycinergic crossover inhibition onto the PV5 RGC is the All, which receives GABAergic input, a required element in my proposed circuit (Bloomfield and Xin, 2000; Zhou and Dacheux, 2004). Further, a sustained $\mathrm{GABA}_{\mathrm{A}}$ ergic current has been observed in mouse ON $\alpha$ and OFF $\alpha_{\text {Sustained }}$ RGCs (Di Macro et al., 2013; Murphy and Rieke, 2006) and a similar, albeit unidentified inhibitory input, was observed in guinea pig OFF $\alpha_{\text {Transient }} R G C s$ (Manookin et al., 2008). Finally, I found no change in GABAergic signaling in PV5 $5_{\text {Glra1-KD }}$ compared to PV5sc when I examined the transcription of GABA-related genes using RNA microarray and deep sequencing strategies (data not shown).

\section{GlyRa1-mediated Crossover Inhibition Directly onto PV5 RGC Improves the Fidelity of RGC Responses}

In the retina, glycinergic inhibition has been primarily associated with an On-to-Off crossover pathway, which appears to: enhance excitatory/inhibitory responses of OFF RGCs to luminance decrement/increment (Buldyrev et al., 2012; Buldyrev and Taylor, 2013; Demb and Singer, 2012; Liang and Freed, 
2010; Manookin et al., 2008; Murphy and Rieke, 2006; Nobles et al., 2012;

Roska et al., 2006), regulate contrast gain control (Beaudoin et al., 2008), contribute to the linearity of the retinal output (Molnar et al., 2009) and shape RGCs responses to specific visual stimuli (Cafaro and Rieke, 2013; Münch et al., 2009).

Within the IPL On-to-Off crossover inhibition has been observed both at the dendrites of OFF RGCs, contributing to an inhibitory conductance, and at the terminals of OFF CBCs, contributing to an excitatory conductance in the OFF RGCs (Manookin et al., 2008; Molnar and Werblin, 2007; Münch et al., 2009; Murphy and Rieke, 2006; Roska et al., 2006). Although these studies inferred a role for glycinergic inhibition at both levels of crossover inhibition, the lack of a subunit specific glycine antagonist made it difficult to directly isolate synapsespecific glycinergic regulation and separate it from GABAergic regulation at the same synaptic level. As a consequence, the relative contribution of modulation at each input level OFF RGC visual activity remains unclear.

For example, direct inhibition is the major drive to OFF $\alpha_{\text {Transient }} R G C$ under rod-dominant conditions or when both rods and cones are active (Manookin et al., 2008; Murphy and Rieke, 2006). Other studies under similar light levels, suggest crossover modulation at the CBC level and its control of excitation in OFF RGCs is critical (Pang et al., 2003; van Wyk et al., 2009). Moreover, the contribution of excitation vs. inhibition appears to change with increasing contrast (Manookin et al., 2008). By eliminating GlyRa1 expression in PV5 RGCs, I show that glycinergic crossover inhibition at the RGC level is critical for regulating 
cone-driven responses within the RF center. Specifically, GlyRa1 inputs lower the spontaneous spiking activity to improve signal to noise ratio in PV5 RGCs. GlyRa1 inputs reduce/eliminate rebound excitation at luminance increment, as well as enhance the excitation response at luminance decrement via disinhibition. Together these mechanisms enhance the fidelity of OFF $\alpha_{\text {Transient }}$ RGC RF center response relative to its dendritic stratification and maintain its ability to primarily encode luminance decrements. 


\section{CHAPTER V}

\section{SUBUNIT SPECIFIC EXPRESSION OF GLYCINE RECEPTOR ACROSS MORPHOLOGICALLY IDENTIFIED MOUSE RETINAL GANGLION CELLS}

\section{Introduction}

The retina is composed of numerous circuits that are tailored to encode spatial, temporal and chromatic aspects of the environment. As the output neuron of the retina, different types of retinal ganglion cells represent the culmination of these circuits and form selective information channels tuned to various aspects of the visual scene (Azeredo da Silveira and Roska, 2011; Dunn and Wong, 2014).

Within these retinal circuits, the diversity of both inhibitory interneurons and their postsynaptic receptors significantly contribute to the differential RGC visual coding properties (MacNeil and Masland, 1998; Menger et al., 1998; Lin and Masland, 2006; Werblin, 2011). Glycine receptors mediate approximately half of the inhibitory modulation within the inner retina, with broad expression in RGCs and their presynaptic cells, such as bipolar cells and amacrine cells (Pourcho, 1996; Wässle et al., 2009; Zhang and McCall, 2012). Synaptic GlyRs 
are pentameric structures thought to be composed of three $\beta$ subunits and two $\alpha$ subunits of a single type (reviewed in Betz and Laube, 2006; Dutertre et al., 2012). There is only one type of $\beta$ subunit in GlyRs. In contrast, there are four types of GlyR $\alpha$ subunit ( $\alpha 1,2,3$ and 4$)$ and all are expressed differentially across the sublaminae of the inner plexiform layer of mouse retina (Wässle et al., 2009). GlyRs containing individual a subunits display distinct kinetic properties and their localization is known on several retinal cell classes and subclasses (Wässle et al., 2009). GlyRa1 has the fastest decay kinetics (Table 1.2) and is known to mediate inhibition in rod and OFF cone BCs, as well as A-type RGCs (Majumdar et al., 2007; Ivanova et al., 2005). GlyRa3 is the second fastest and is expressed on All ACs (Weiss et al., 2008). GlyRa2 and GlyRa4 are significantly slower and are localized on dendrites of some narrow field ACs (Weiss et al., 2008), a variety of displaced widefield ACs and ON starburst ACs (Majumdar et al., 2009).

Except for A-type RGCs (A1, A2-ON and A2-OFF) which express GlyRa1 (Majumdar et al., 2007), the type of GlyR a subunit expressed in different RGCs remains largely unknown. This gap in our knowledge restricts our understanding of why four different $\alpha$ subunits with different kinetics are expressed as separate inhibitory elements in the retinal circuit. Before the new approach I developed and used in Chapter IV, e.g. AAV-RNAi strategy can be applied to a large-scale study of glycinergic subunit specific inhibition of RGC function, it is critical to characterize the specific expression of GlyR a subunit across morphologically identified RGCs. To this end, I identified the GlyR a subunit(s) expressed in eight 
morphologically identified RGC types, using the reporter mouse line PvalbCre $\times$ ThyStp-EYFP $\left(P V^{\text {cre }}\right)$. The retina of this mouse has eight morphological RGC types that express YFP and have different visual responses within their RF center (Table 5.1). These RGCs have been denoted as PV0 - PV7 (Farrow et al., 2013). In a representative sample of most of these RGC types, I measured decay kinetics of pharmacologically isolated glycinergic spontaneous IPSCs and examined the co-localization incidence of GlyR a subunit expression on the same RGC dendrites. My results indicate striking diversity in the GlyR a subunits across morphologically identified RGCs. Some types of RGCs predominantly express one type of GlyR a subunit. For example, four PV RGCs (PV1, 2, 5 and 6) express GlyRa1. In contrast, some PV RGCs express more than one type of GlyR a subunit. Among my sample, when more than one is expressed the subunits appear to be matched in terms of their temporal kinetics. For example, PV0 RGCs express the slower subunits, GlyRa2 and GlyRa4; whereas PV4 RGCs express the two faster subunits, GlyRa1 and GlyRa3. In contrast to the others, PV7 RGCs predominantly receive not only GlyRa3- but also GlyRa2mediated synaptic input. Thus, the results support the existence of subunit and cell specific glycinergic regulation within the retina and provide important new information about subunit selective synaptic input to identified RGCs. This information forms a solid framework for future work to investigate the role of subunit-specific glycinergic modulation in the retina, which may be more broadly found throughout the rest of the central nervous system. 
Table 5.1 Types of RF center response across PV RGC type (summarized from Farrow et al., 2013).

\begin{tabular}{c|l}
\hline RGC Cell Type & \multicolumn{1}{|c}{ RF Center Response } \\
\hline PV0 & Transient ON \& Transient OFF \\
PV1 & Sustained ON \\
PV2 & Transient ON \\
PV3 & Sustained ON \& Transient OFF \\
PV4 & Transient OFF \\
PV5 & Transient OFF \\
PV6 & Sustained OFF \\
PV7 & Transient OFF \\
\hline
\end{tabular}

\section{Results}

\section{Morphological Characterization of PV RGCs}

In $P V^{\text {cre }}$ mouse retina, whole cell recordings characterized sIPSCs from the eight types of YFP positive RGCs. The exact morphological identity was confirmed using immunohistochemistry and confocal microscopy on recorded RGCs that were filled with neurobiotin or Lucifer Yellow included in the intracellular solution. The majority (136 of $146,93 \%$ ) of recorded and filled RGCs could be classified into the eight already identified PV morphological RGC types (PV0-PV7; Farrow et al., 2013; c.f. Figure S1). I also used the same dendritic morphological criteria: shape, size and stratification pattern within the IPL, as 
defined by their relationship to the ChAT bands (Figure 5.1). When $P V^{c r e}$ mice were crossed and backcrossed to GlyR knockout mice, the morphology of YFP positive RGCs was similar compared to WT across PV RGC types. The morphology of each PV RGC type is described in detail in the following corresponding sections. Because only one PV3 RGC was recorded in my study, it is not discussed in the result. About $11 \%$ of the recorded YFP-positive RGCs could not be matched to any PV0 - PV7 RGC types. These RGCs were heterogeneous in morphology and were excluded from the analysis.
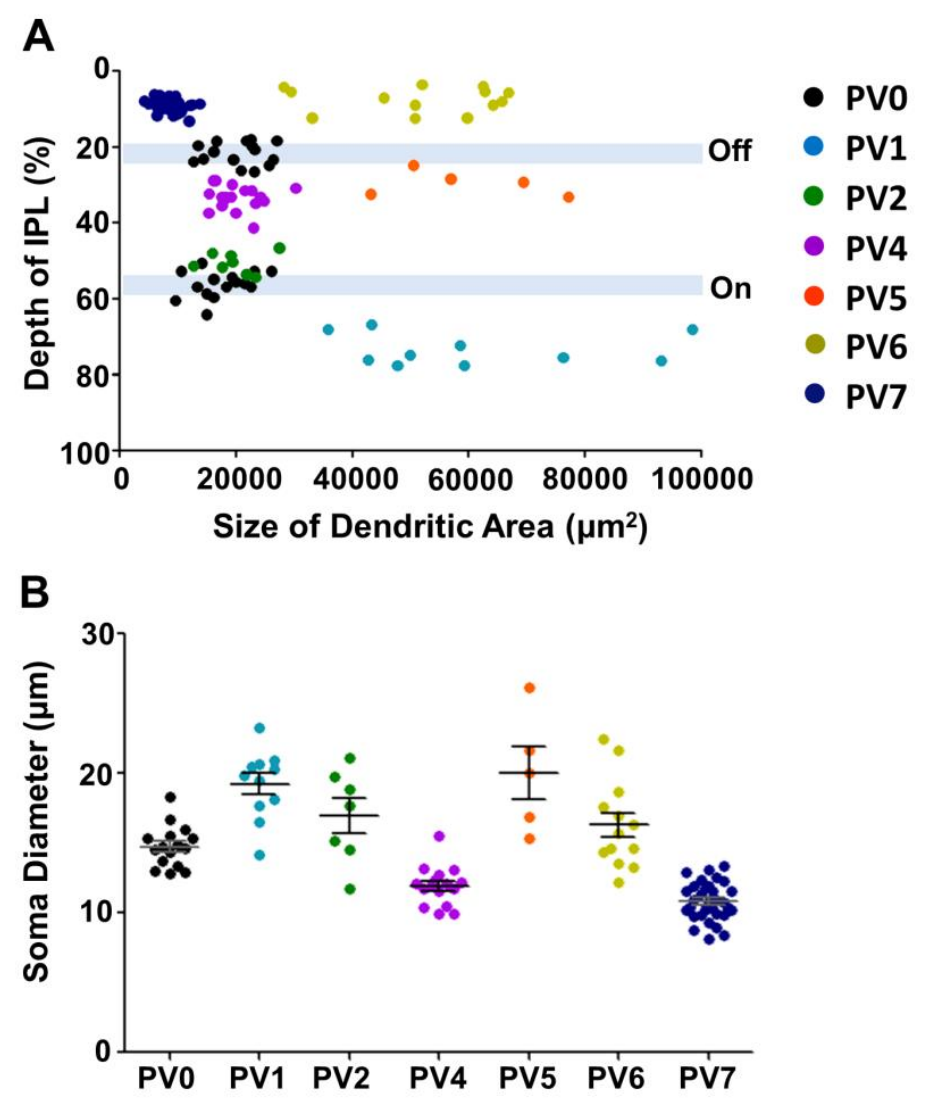

Figure 5.1 Summary of morphological quantification of recorded PV RGCs.

Using conventions similar to Farrow et al., 2013, the dendritic area size of each PV RGC is plotted against their dendritic stratification depth within the inner 
plexiform layer in $(\mathbf{A})$. The two blue horizontal bands indicate the position of On and Off ChAT bands ( $n=27)$. The soma diameter of each PV RGC is plotted in (B). Each RGC type is color coded and each symbol represents a single RGC. PV0, n=15; PV1, n=10; PV2, n=8; PV4, n=18; PV5, n=5; PV6, n=13; PV7, n=42.

\section{PV1, 2, 5 and 6 RGCs Express GlyRa1 Subunits and Synaptic Currents}

In addition to PV5 (transient OFFa RGCs), which were described previously (Zhang et al., 2014; also see in Chapter IV), three other RGC types (PV1, PV6 and PV2) predominantly express GlyRa1.

The PV1 has been described as the sustained ONa RGC. A luminance increment within their RF center evokes a sustained response that matches the duration of the stimulus (Farrow et al., 2013, c.f. Figure 2; Pang et al., 2003). PV1 RGCs have large soma diameters (Figure 5.1B). Their large dendritic fields (Figure 5.1A) stratify on the inner side of the ON ChAT band (Figure 5.2Ai) and proximal to the ganglion cell layer.

The PV6 RGC has been described as the sustained OFFa RGC. A luminance decrement within their RF center evokes a sustained response that matches the duration of the stimulus (Farrow et al., 2013, c.f. Figure 2; Pang et al., 2003). As PV1 RGCs, PV6 RGCs also have large soma diameters and large dendritic fields (Figure 5.1), but stratify on the outer side of the OFF ChAT band (Figure 5.2Bi), very near to the inner nuclear layer. 
The PV2 RGC is a type of ON RGC. Unlike PV1 and PV6 RGCs, the physiological properties of PV2 have not been investigated well, except that a luminance increment within their RF center evokes a transient response, shorter than the duration of the stimulus (Farrow et al., 2013, c.f. Figure 2). PV2 RGCs also have large soma diameters (Figure 5.1B). Their dendritic fields are significantly smaller than PV1 (Figure 5.1A; t-test, $p<0.0001$ ) and they stratify along the ON ChAT band (Figure 5.2Ci). Morphologically PV2 is similar with both the B3i (Sun et al., 2002) and $\mathrm{G}_{6}$ RGCs (Völgyi et al., 2012).

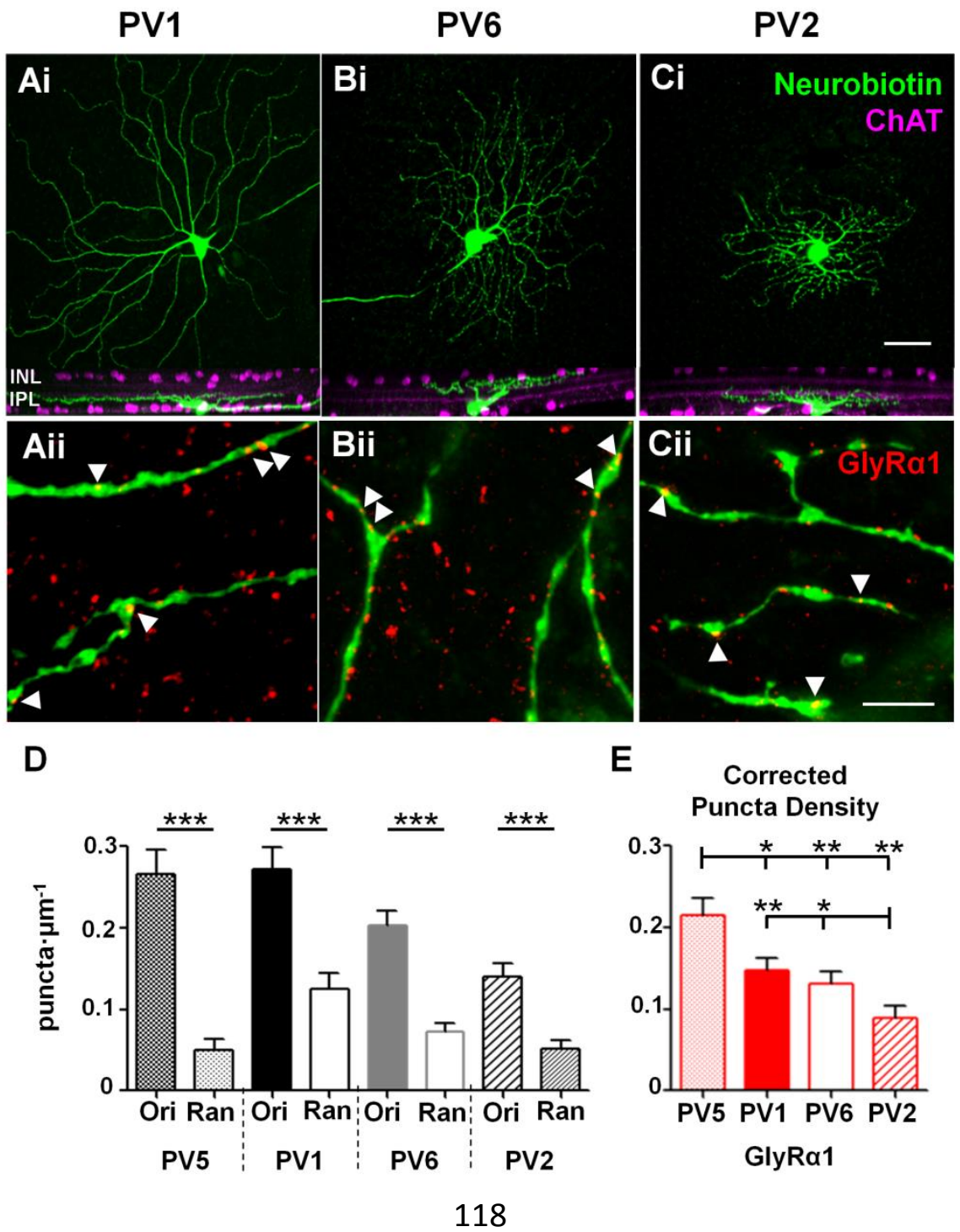


Figure 5.2 The GlyRa1 subunit is expressed on PV1, PV6 and PV2 RGCs. (Ai, $\mathbf{B i}, \mathbf{C i})$ Top, representative en face wholemount confocal images of neurobiotin labeled (green) PV1, 6 and 2 RGC ; Bottom, rotated view of the same RGC that show its dendritic stratification relative to the bands formed by the processes of the cholinergic amacrine cells (ChAT bands, magenta). (Aii, Bii, Cii) Representative images showing double labeling of GlyRa1 (red) and neurobiotin labeled RGC dendrites (green). The white arrows indicate subsets of co-localized GlyRa1 puncta on the dendrites of each type of RGCs. (D) The original (Ori) density of co-localized GlyRa1 puncta in each type of RGCs was significantly higher than the random density (Ran), suggesting prominent expression of GlyRa1 in PV5 ( $n=4, N=8), P V 1$ ( $n=8$ cells, $N=15$ areas), PV6 $(n=9, N=16)$ and PV2 ( $n=8, N=12)$. (E) The corrected puncta density on the dendrites of each RGC type. Abbreviations: INL, inner nuclear layer; IPL, inner plexiform layer. Scale bar $=40 \mu \mathrm{m}$ in Ai, Bi \& Ci; $5 \mu \mathrm{m}$ for Aii, Bii \& Cii.

Picrotoxin $(15 \mu \mathrm{M})$ and TPMPA $(50 \mu \mathrm{M})$ block $\mathrm{GABA}_{\mathrm{A}}$ and $\mathrm{GABA}_{\mathrm{C}}$ receptor mediated currents and were used to isolate and characterize the glycinergic sIPSCs in these RGCs. Like PV5 (Chapter IV), PV1, 6 and 2 RGCs all received prominent sIPSCs in the presence of PTX and TPMPA (Figure 5.3A) and all were eliminated when strychnine was subsequently added to the bath, suggesting that they are glycinergic (Figure 5.3A). Similar with previous studies in RGCs and ACs (Majumdar et al., 2007, 2009; Veruki et al., 2007), all sIPSC 
decays across RGC types in my study could be well fit with a single exponential function (Figure 5.3A-C) and this function was used to derive their decay time constant (TDecay). For each recorded cell, there was no correlation between rise time $\left(10-90 \%\right.$ of peak) and amplitude or between amplitude and $\mathrm{T}_{\text {Decay }}$ regardless of different size and shape of dendritic field (data not shown). These results suggest that the decay is not affected by dendritic filtering. Within each group of PV RGCs, the mean $T_{\text {Decay }}$ was similar regardless of whether I averaged the mean value of each cell or averaged the value of every single event from all the cells. All the values reported here are the averages of all the single events. The

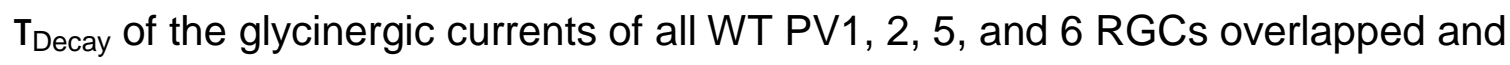
ranged from 1 to $11 \mathrm{~ms}$ with >95\% events faster than $6 \mathrm{~ms}$ (Figure 5.3D\&E; Kolmogorov-Smirnov test, all $p>0.05$ ). The mean $\mathrm{T}_{\text {Decay }}$ of all events was $\sim 3 \mathrm{~ms}$. This result suggests that all four types of RGC receive glycinergic sIPSC with fast decay kinetics that match those mediated by GlyRs containing the a1 subunit (Gill et al., 2006; $\mathrm{T}_{\text {Decay }}=2.9 \mathrm{~ms}$ at $34^{\circ} \mathrm{C}$ ).

GlyRa1 subunits expression extends across the entire IPL, although it is found in higher density in the Off sublamina (Figure 1.6). Consistent with the fast kinetics of their glycinergic IPSCs, the immunohistochemical analysis also showed that similar to PV5, WT PV1, 6 and 2 RGCs had significant expression of GlyRa1 on their dendrites. The coincidence rate of GlyRa1 puncta associated with the dendrite was significantly higher than an estimate of random coincidence (Figure 5.2; paired t-test, all $p<0.0001$ ). The overall density of GlyRa1 expression 
on PV1 and PV6 were similar and both were significantly higher than PV2 (Figure 5.2C-E; t-tests, PV1, $p=0.01 ; P V 6, p=0.02$ ). The density in PV5 was significantly higher than PV1, 6 and 2 (t-tests, PV1, $p=0.02 ; \mathrm{PV} 6, p=0.006 ; \mathrm{PV} 2, p=0.001$ ).

Because my results are consistent with a previous study that showed two A-type RGCs (A1 and A2-OFF) that are morphologically similar to PV1 and PV6 RGCs also predominantly express GlyRa1, I did not examine expression of other GlyR a subunits (Majumdar et al., 2007). In conclusion, previously published and these results show that WT PV1, PV2, PV5 and PV6 express GlyRa1, which mediates the majority of their synaptic glycinergic input. 
Ai

Aii

Aiii

Control

PTX+TPMPA

PTX+TPMPA

+strychnine

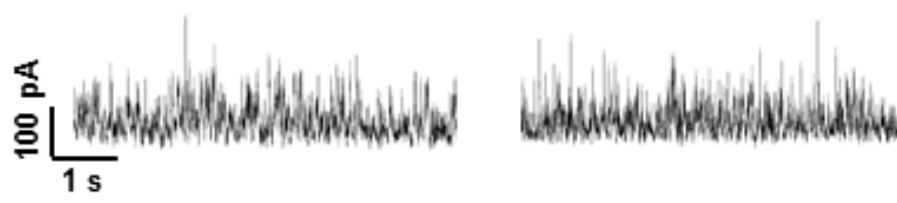

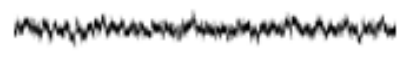

B

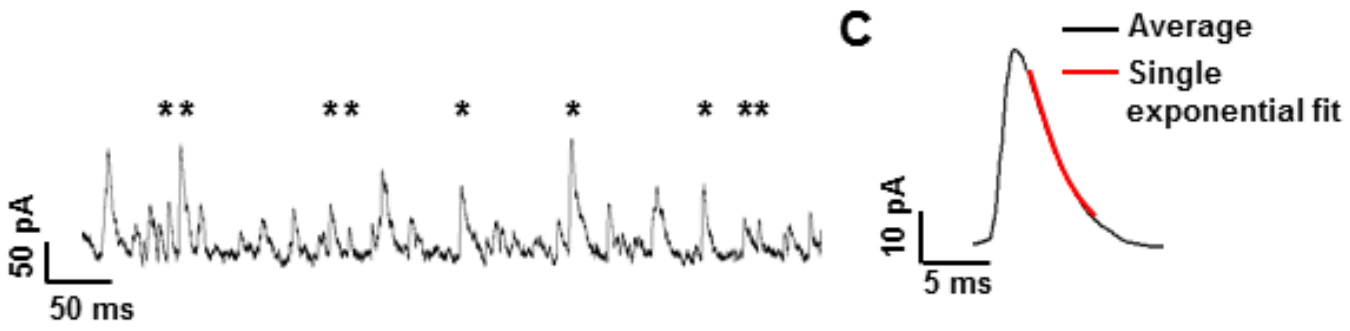

D

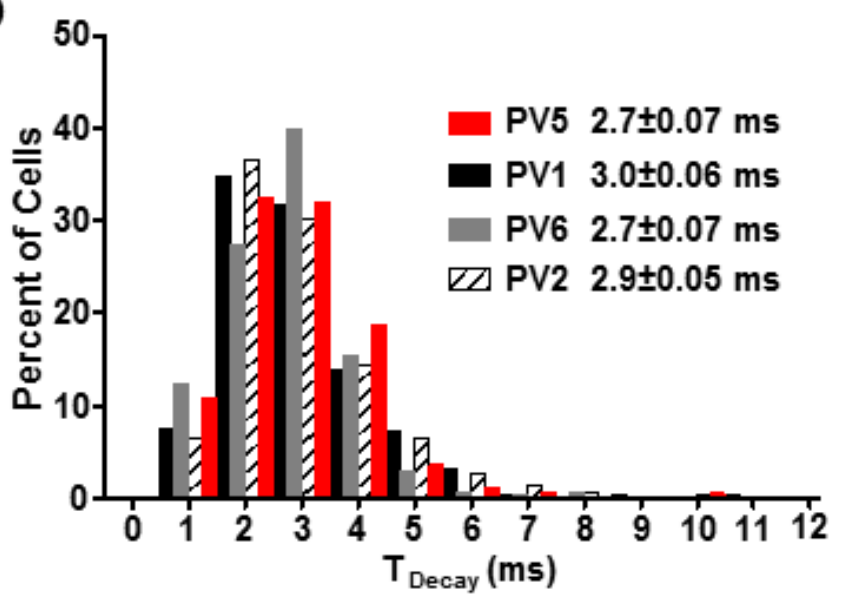

$\mathbf{E}$

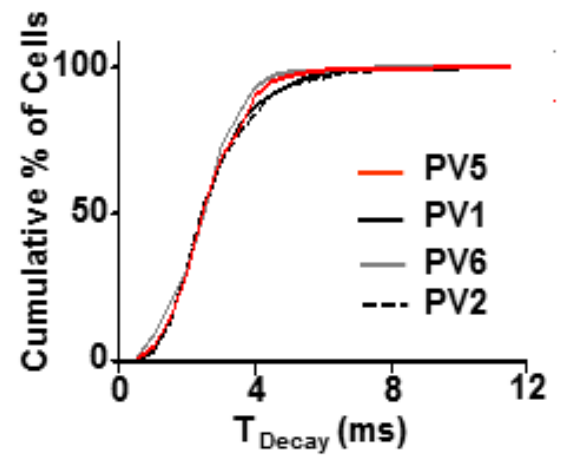

Figure 5.3 PV1, 2, 5 and 6 RGCs receive GlyRa1-mediated spontaneous inhibitory postsynaptic currents (sIPSC). (A) Representative sIPSCs recorded from a PV2 RGC under control condition (Ai), in presence of picrotoxin (PTX, 15 $\mu \mathrm{M})$ and TPMPA $(50 \mu \mathrm{M})$ without (Aii) and with (Aiii) strychnine (10 $\mu \mathrm{M})$. (B) sIPSCs recorded from the same cell in Aii at higher temporal resolution. Stars indicate sIPSCs that met my criterion (see methods) and were used in the analysis. (C) Average waveform of the sIPSCs from the same recording in Aii that met the criterion (134 events). The decay constant was $2.4 \mathrm{~ms}$ with a single exponential fitting (10-90\% of decay). The $10-90 \%$ of peak rise time was $1.1 \mathrm{~ms}$. 
(D) The frequency of $\mathrm{T}_{\text {Decay }}$ of sIPSCs in WT PV5 ( $\mathrm{n}=4$ cells, $\mathrm{N}=166$ events), PV1 $(n=8, N=568)$, PV6 $(n=6, N=273)$ and PV2 RGCs $(n=8, N=634)$. (E) The cumulative frequency distribution of sIPSCs $\mathrm{T}_{\text {Decay }}$ of WT PV5, PV1, PV6 and PV2 RGCs overlap with each other, suggesting similar distribution.

\section{PV4 RGCs Express both GlyRa1 and GlyRa3 Subunits and Synaptic Currents}

The PV4 RGC is a type of transient OFF RGCs. The physiological properties of PV4 have not been well investigated, except that a luminance decrement within their RF center evokes a transient response that is shorter than the duration of the stimulus (Farrow et al., 2013, c.f. Figure 2). PV4 RGCs have small somas (Figure 5.1B) and their dendritic fields stratified at the same IPL depth as the PV5 RGC, which has a significantly larger dendritic field ( $p=0.002$; Figure 5.1, 5.8A). The morphology of the PV4 RGC resembles both the B1 (Sun et al., 2002) and $\mathrm{G}_{4}$ RGCs (Völygi et al., 2009).

WT PV4 RGCs received glycinergic sIPSCs (Figure 5.5A\&B) whose $\mathrm{T}_{\text {Decay }}$ of ranged from $1 \mathrm{~ms}$ to $20 \mathrm{~ms}$, with a mean of $5.2 \pm 0.1 \mathrm{~ms}$ (Figure $5.5 \mathrm{C} \& \mathrm{E}$ ). The mean $\mathrm{T}_{\text {Decay }}$ in WT PV4 RGCs was slower compared to PV1, 2, 5 and 6 RGCs that express GlyRa1 (Kolmogorov-Smirnov test, all $p<0.001$ ) and faster than GlyRa3-mediated sIPSCs ( 11 ms; Table 1.5). These results show that WT PV4 
RGCs are likely to express more than one type of GlyR a subunit that have fast kinetics, such as GlyRa1 and GlyRa3.

In support of this hypothesis, the frequency of glycinergic sIPSCs recorded from Glra3 ${ }^{-/}$PV4 RGCs was significantly lower (Figure 5.5D; t-test, $p=0.03$ ) and their $\mathrm{T}_{\text {Decay }}$ faster compared to WT (Figure 5.5E-G; Kolmogorov-Smirnov test, $p<0.001$ ). More than $90 \%$ of the remaining sIPSCs in Glra3 ${ }^{-/}$PV4 RGCs are fast events with $\mathrm{T}_{\text {Decay }}<6 \mathrm{~ms}$, suggesting GlyRa1-dominant currents. However, the $\mathrm{T}_{\text {Decay }}$ of the remaining sIPSCs in Glra3 ${ }^{-/}$PV4 RGCs was slower than GlyRa1dominant mean $\mathrm{T}_{\text {Decay }}$ of $\mathrm{PV} 1,2,5$ and 6 (Kolmogorov-Smirnov test, all $p<0.001$ ). This could mean that there also is low expression of other GlyR a subunits with slower kinetics. Because the sIPSC in GIra2 ${ }^{-/}$PV4 RGCs had the same frequency and same mean $\mathrm{T}_{\text {Decay }}$ compared to $\mathrm{WT}$, I do not think the remaining current are mediated by GlyRa2 in PV4 RGCs.

Consistent with the electrophysiological data that indicated sIPSC mediated by both GlyRa1 and GlyRa3 in WT PV4 RGCs, there were immunoreactive puncta GlyRa1 and GlyRa3 that co-localized with the PV4 dendrites (Figure 5.4B\&C). However, only GlyRa1 showed significant colocalization (Figure 5.4D\&E). This failure to demonstrate co-localization of GlyRa3 could be due to a low expression level and further analysis will require a more sensitive assey.

In WT, GIra2 ${ }^{-/}$and Glra3 ${ }^{-/}$retina, I recorded from RGCs with PV4 morphology (WT, $n=1 ;$ Glra2 ${ }^{-/}, n=2 ;$ Glra3 ${ }^{-/}, n=1$ ) but with much higher sIPSCs 
frequency $(>18 \mathrm{~Hz})$ than in the majority of PV4 RGCs. These cells had sIPSCs with GlyRa1-dominated decay kinetics (mean $\mathrm{T}_{\text {Decay }}<4 \mathrm{~ms}$ ). Although they could not be morphologically distinguished from the other PV4 RGCs, these RGCs might represent a functionally different group. More studies are required to further classify these PV4 morphology RGCs, such as specific light stimulation.
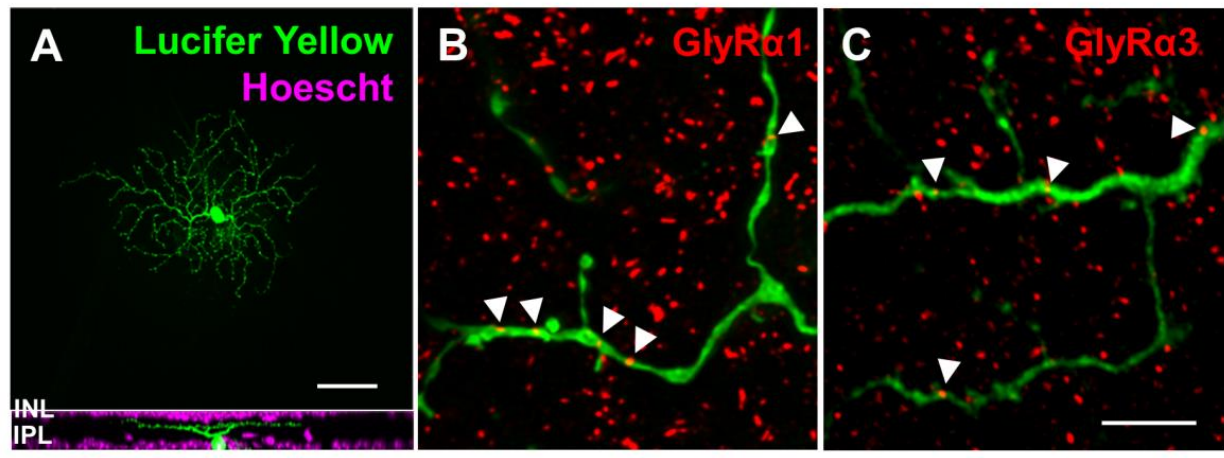

D

PV4

$\mathbf{E}$
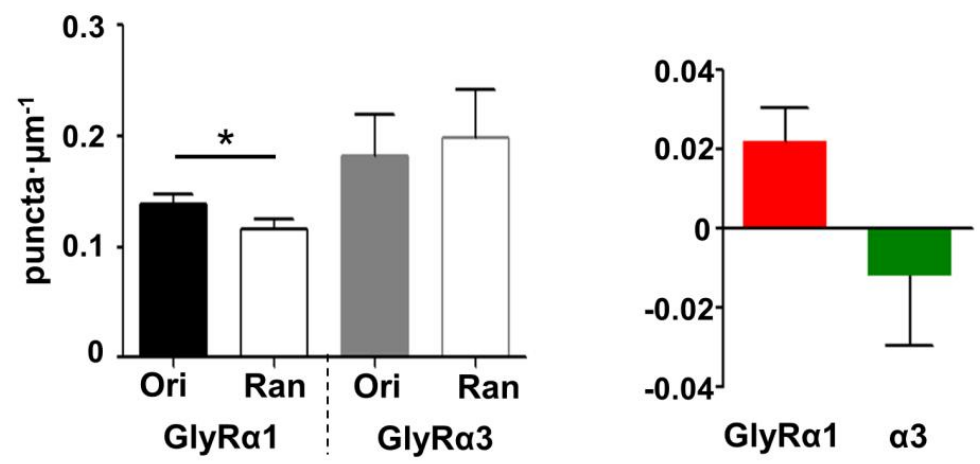

Figure 5.4. PV4 RGCs significantly express GlyRa1 subunits on their dendrites. (A) Top, representative en face wholemount confocal images of Lucifer Yellow labeled PV4 RGC (green); Bottom, rotated view of the same RGCs that show their dendritic stratification pattern relative to the inner plexiform layer defined by Hoescht nuclear staining (magenta). (B\&C) Representative images showing double staining of GlyRa1 or GlyRa3 (red) and labeled PV4 RGC 
dendrites (green). The white arrows indicate subsets of co-localized GlyRa puncta on the dendrites of each type of RGCs. (D) Comparisons of the original (Ori) and random (Ran) density of co-localized GlyRa1 ( $\mathrm{n}=6$ cells, $\mathrm{N}=11$ areas) and GlyRa3 ( $n=3, N=5)$ in PV4 RGCs. (F) The corrected puncta density of GlyRa1 and GlyRa3 on the dendrites of PV4 RGCs. Abbreviations: INL, inner nuclear layer; IPL, inner plexiform layer. Scale bar $=40 \mu \mathrm{m}$ in $A ; 5 \mu \mathrm{m}$ in B\&C. 
Ai

Aii

Aiii

Control

PTX+TPMPA

PTX+TPMPA

+ Strychnine

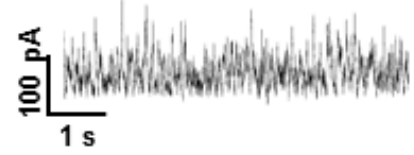

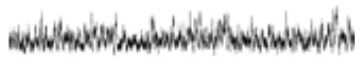

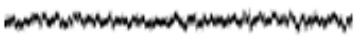

B

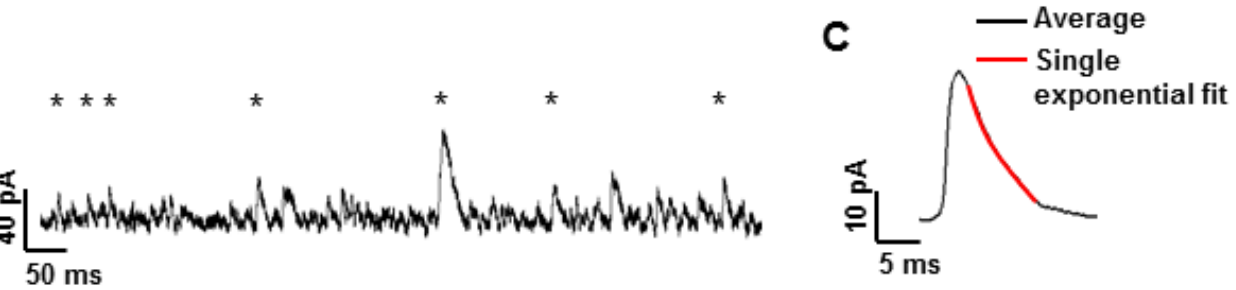

D

E
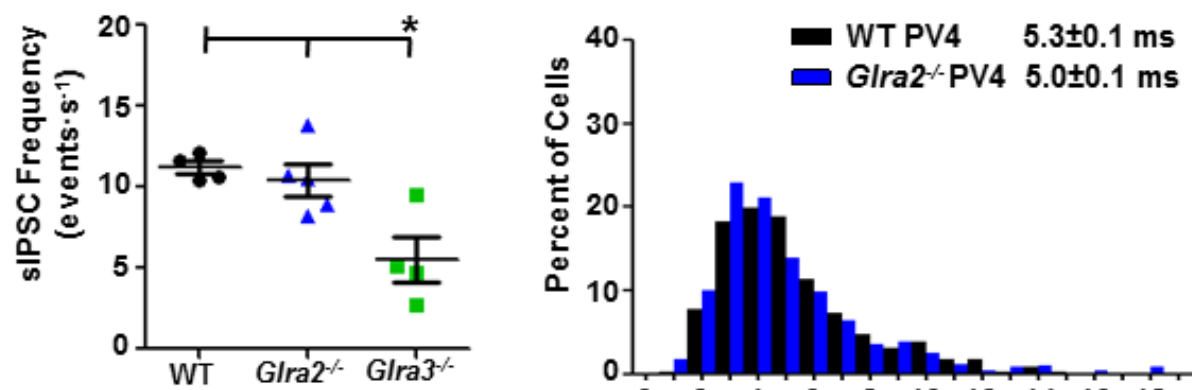

F

G

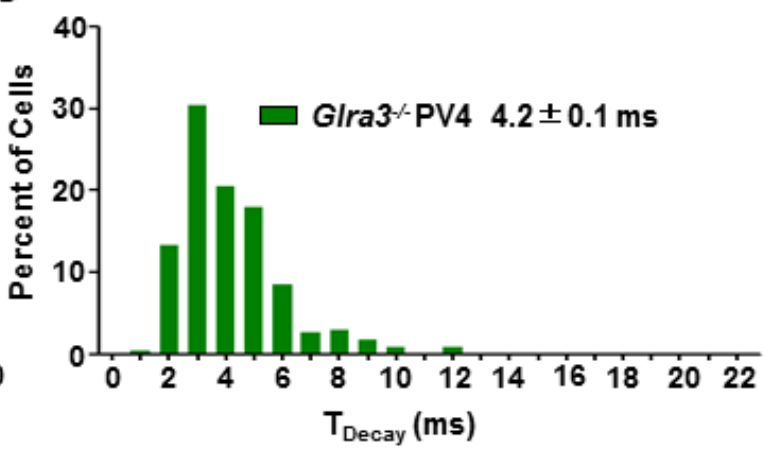

Figure 5.5. PV4 receive sIPSCs that are mediated both by GlyRa1 and

GlyRa3. (A) Representative sIPSCs recorded from a PV4 RGC under control condition (Ai), in presence of picrotoxin (PTX, $15 \mu \mathrm{M})$ and TPMPA $(50 \mu \mathrm{M})$ without (Aii) and with (Aiii) strychnine $(10 \mu \mathrm{M})$. (B) sIPSCs recorded from the same cell in Aii at higher temporal resolution. Stars indicate sIPSCs that met my 
criterion (see methods) and were used in the analysis. (C) Average waveform of the sIPSCs from the same recording in Aii that met the criterion (62 events). The decay constant was $5.1 \mathrm{~ms}$ with a single exponential fitting (10-90\% of decay). The $10-90 \%$ of peak rise time was $1.2 \mathrm{~ms}$. (D) The frequency of glycinergic sIPSCs of Glra3 ${ }^{-\sim}(n=4)$ PV4 RGCs are lower than WT PV4 RGCs $(n=4)$, whereas Glra2 ${ }^{--}$PV4 $(n=5)$ are the same with WT. (E) The frequency distribution of the sIPSC TDecay of WT PV4 ( $n=4$ cells, $N=361$ events) is similar to Glra2 ${ }^{-/}(n=5$, $\mathrm{N}=532$ ) but significantly different compared to (G) $\mathrm{Glra3}^{--}$( $\left.\mathrm{n}=4, \mathrm{~N}=234\right) \mathrm{PV7}$ RGCs, which is faster. (F) Cumulative frequency distribution of SIPSC TDecay $_{\text {of }}$ PV4 RGCs across genotype.

\section{PV7 RGCs Express both GlyRa3 and GlyRa2 Subunits and Synaptic Currents}

The PV7 RGC is a type of transient OFF RGC. A luminance decrement within their RF center evokes a transient response that is shorter than the duration of stimulus (Farrow et al., 2013; c.f. Figure 2). PV7 RGCs have small soma diameters (Figure 5.1B) and a distinct asymmetric wedge-shaped dendritic field; all of the dendrites are found on one side of the cell soma. PV7 RGCs usually have 1-3 primary dendrites that extend from the soma and branch in the middle of IPL and further ramify into a bushy dendritic field that ascends to the outermost Off sublamina of IPL, proximal to the INL (Figure $5.1 \& 5.6 \mathrm{~A}$ ). In a previous study (Farrow et al., 2013), PV7 has been suggested to be synonymous 
with the JAMB RGC, which is a direction selective cell sensitive to upward motion (Kay et al., 2011; Kim et al., 2008, 2010). However, the size of the dendritic field of PV7 RGCs in my sample and in Farrow et al. (2013) is significantly smaller than the JAMB RGCs (average dendritic area size $\sim 10.000 \mu \mathrm{m}^{2}$ vs. $\sim 35,000 \mu \mathrm{m}^{2}$; Kim et al., 2010), indicating that PV7 and JAMB are more likely different types of RGC. Morphologically the PV7 RGC is similar to $G_{15}$ (Völgyi et al., 2012) and the monostratified cluster 6 RGC (Badea and Nathans, 2004). More studies are required to characterize the physiological properties of PV7.
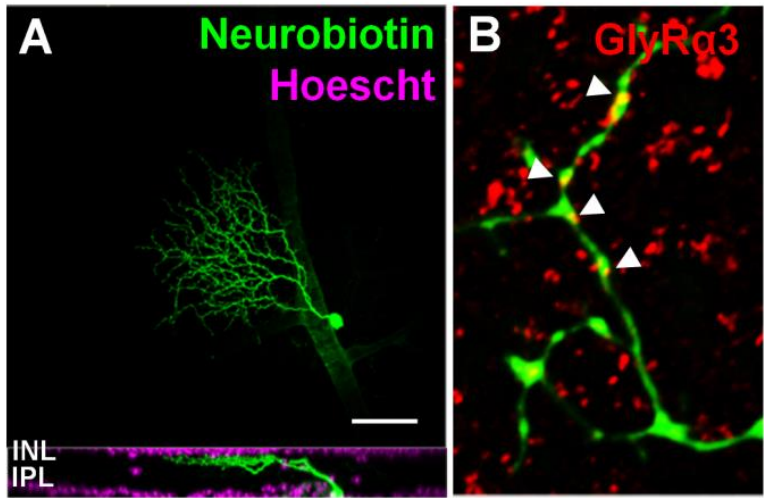

$E$

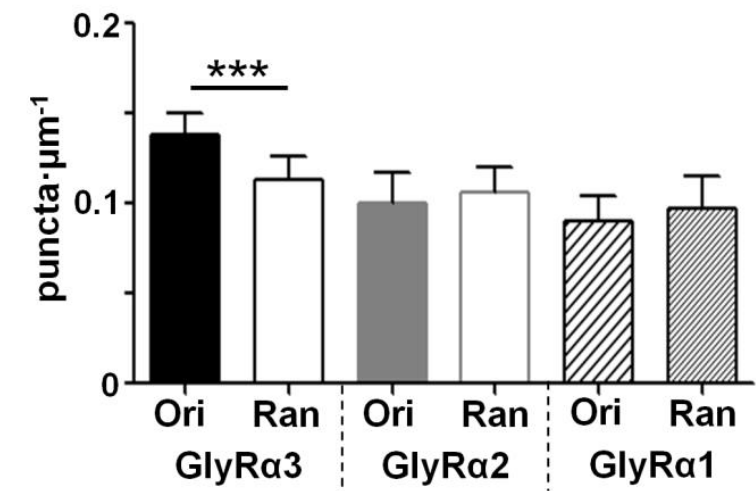

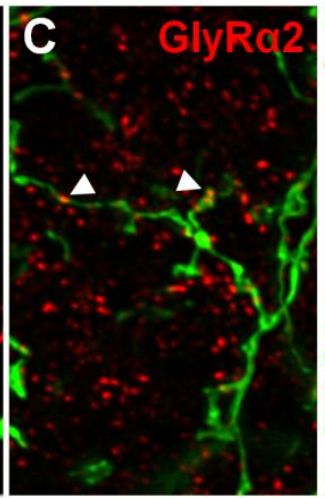

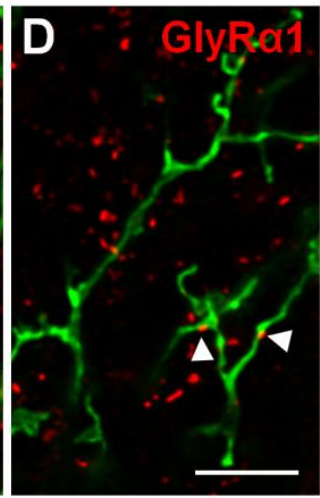

$\mathbf{F}$

Corrected Puncta Density

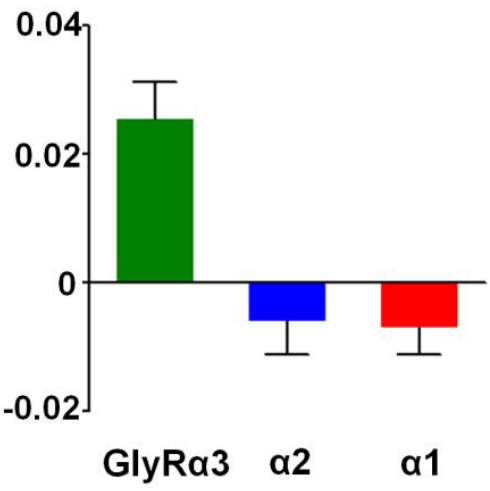


Figure 5.6. PV7 RGCs significantly express GlyRa3 subunits on their

dendrites. (A) Top, representative en face wholemount confocal images of neurobiotin labeled PV7 RGC (green); Bottom, rotated view of the same RGCs that show the dendritic stratification within the inner plexiform layer defined by Hoescht nuclear staining (magenta). (B-D) Representative images of double labeling of GlyRa3, GlyRa2,or GlyRa1(red) and neurobiotin labeled PV7 RGC dendrites (green) in the On and Off sublamini. The white arrows indicate subsets of co-localized GlyRa puncta on the dendrites of PV07RGCs. (E) The comparisons of the original (Ori) and random density (Ran) density of colocalized GlyRa3 ( $n=9$ cells, $N=11$ areas), GlyRa2 $(n=4, N=5)$ and GlyRa1 $(n=9$, $\mathrm{N}=10)$. (F) The corrected puncta density of $\mathrm{GlyRa3}$, GlyRa2 and GlyRa1 on the dendrites of PV7 RGCs. Abbreviations: INL, inner nuclear layer; IPL, inner plexiform layer. Scale bar $=40 \mu \mathrm{m}$ in A; $5 \mu \mathrm{m}$ in B-D.

In the presence of PTX and TPMPA, WT PV7 RGCs receive glycinergic sIPSCs (Figure 5.7A), that could be eliminated by application of strychnine. Single exponential fitting of the isolated glycinergic sIPSCs in WT PV7 RGCs showed a $\mathrm{T}_{\text {Decay }}$ that ranged from 2 to $26 \mathrm{~ms}$ with a mean of $6.6 \pm 0.1 \mathrm{~ms}$ (Figure 5.7). The $T_{\text {Decay }}$ of $W T P V 7 R G C$ s was significantly slower than $T_{\text {Decay }}$ of GlyRa1mediated sIPSCs in PV1, 2, 5 and 6 RGCs. They are also significantly slower than the sIPSCs in PV4 RGCs that are likely a combination of GlyRa1 and $\alpha 3$ (Kolmogorov-Smirnov test, all $p<0.001$ ). Because the $T_{\text {Decay }}$ of PV7 RGCs is much 
faster than GlyRa2- and GlyRa4-mediated current (>20 ms; Table 1.2), that the subunit expressed in WT PV7 RGCs is most likely to be GlyRa3.

In order to test whether GlyRa3 was the main subunit mediating glycinergic input, I recorded sIPSCs in PV7 RGCs in Glra3 ${ }^{-/}$retina. In the absence of GlyRa3 expression, the frequency of glycinergic sIPSCs in Glra3 ${ }^{-/}$ PV7 RGCs was significantly lower compared to WT (Figure 5.7D; t-test, $p<0.0001)$. The remaining sIPSCs could be eliminated by strychnine and had slower $\mathrm{T}_{\text {Decay }}$ compared to WT (Figure 5.7E-G; Kolmogorov-Smirnov test, $p<0.001$ ), suggesting that other GlyR a subunits also are expressed in PV7 RGCs. Consistent with this observation, the absence of GlyRa2 expression also significantly lowered the frequency of glycinergic sIPSCs (Figure 5.7D; t-test, $p=0.001$ ) and slowed the $\mathrm{T}_{\text {Decay }}$ of glycinergic sIPSCs in GIra2 ${ }^{-/}$PV7 RGCs compared to WT (Figure 5.7E-G; Kolmogorov-Smirnov test, $p<0.001$ ). These results suggest that GlyRa2 and GlyRa3 contribute to the glycinergic synaptic input. Although GlyRa2 and GlyRa3 have very different kinetics, the remaining glycinergic sIPSCs in Glra2 ${ }^{-/}$and Glra3 ${ }^{-/}$PV7 RGCs showed similar $\mathrm{T}_{\text {Decay }}$ distributions (Figure 5.7F\&G; Kolmogorov-Smirnov test, $p=0.13$ ). This is an unexpected result. 
Ai

Control

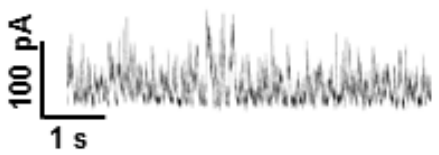

B

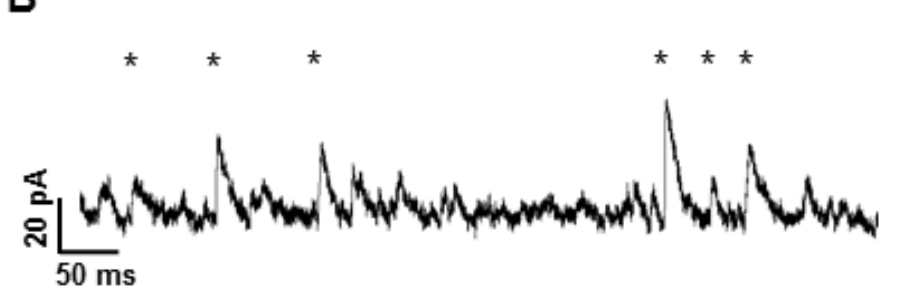

Aiii

PTX+TPMPA

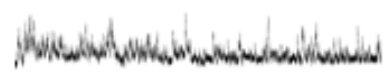

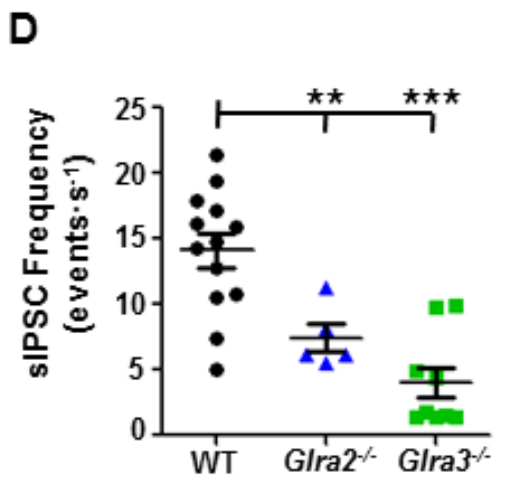

F

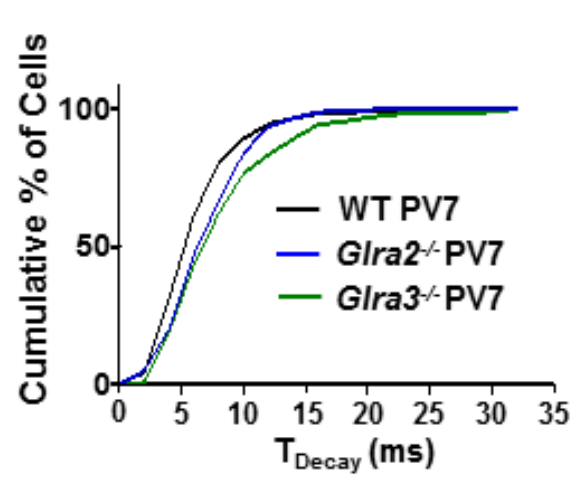

E
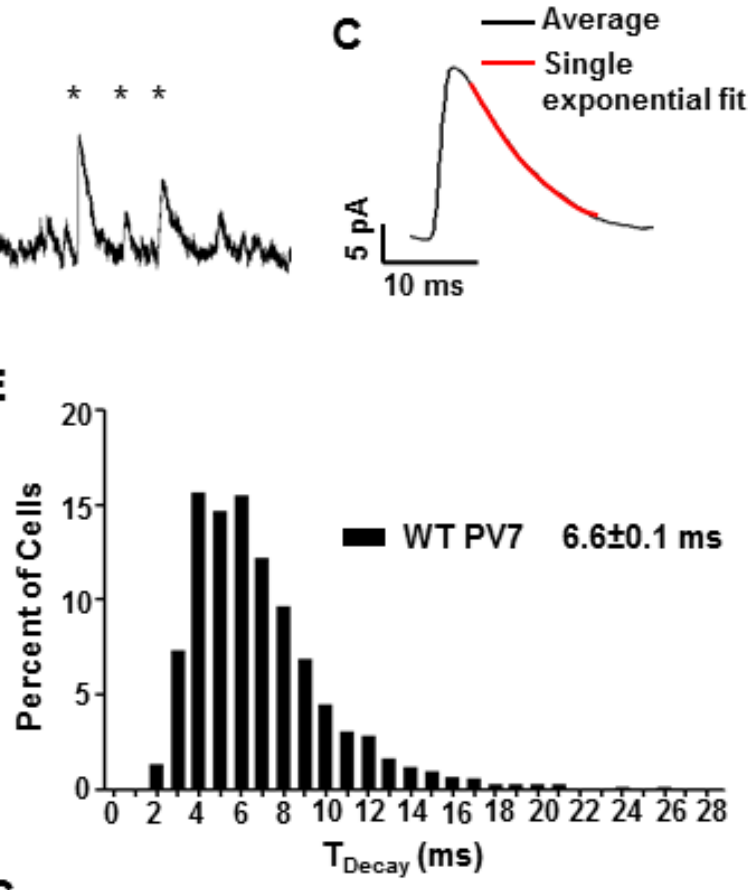

$\mathbf{G}$

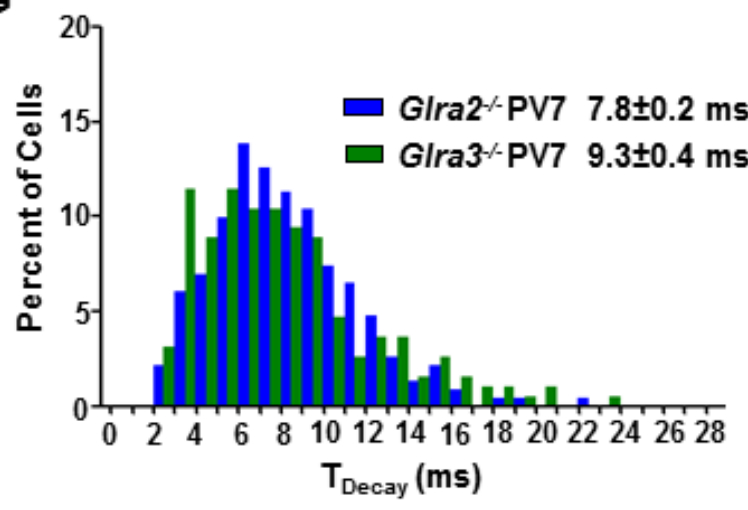

Figure 5.7. PV7 receive sIPSCs mediated by GlyRa3 and GlyRa2. (A)

Representative sIPSCs recorded from a PV7 RGC under control condition (Ai), in presence of picrotoxin (PTX, $15 \mu \mathrm{M})$ and TPMPA $(50 \mu \mathrm{M})$ without (Aii) and with (Aiii) strychnine $(10 \mu \mathrm{M})$. (B) sIPSCs recorded from the same cell in Aii at 
higher temporal resolution. Stars indicate sIPSCs that met my criterion (see methods) and were used in the analysis. (C) Average waveform of the sIPSCs from the same recording in Aii that met the criterion (90 events). The decay constant was $6.7 \mathrm{~ms}$ with a single exponential fitting (10-90\% of decay). The 10$90 \%$ of peak rise time was $1.1 \mathrm{~ms}$. (D) The frequency of glycinergic sIPSCs of Glra2 $^{-/}(n=5)$ and GIra3 ${ }^{-/}(n=9)$ PV7 RGCs were both significantly lower than WT PV7 RGCs ( $n=15)$. (E) The frequency distribution of the sIPSC TDecay of WT PV7 ( $n=15$ cells, $N=760$ events) is significantly different from (G) G/ra2 ${ }^{-/}(n=5, N=231)$ and Glra3 $^{-/}(\mathrm{n}=9, \mathrm{~N}=192)$ PV7 RGCs. (F) Cumulative frequency distribution of sIPSC T Tecay $_{\text {of }}$ PV7 RGCs across genotype.

The expression of GlyRa3 forms four bands within the IPL with the densest band in the Off sublamina which is closer to the ONL than the OFF ChAT band (Figure 1.6). Consistent with the electrophysiological data, my immunohistochemical analysis using the GlyRa3 antibody in WT PV7 RGCs showed significant co-localization on the dendrites (Figure 5.6). In contrast, there was no significant co-localization of GlyRa1 or GlyRa2 on WT PV7 dendrites although immunoactive puncta were found (Figure 5.6). These results suggest that WT PV7 RGCs predominantly express GlyRa3. GlyRa2 is likely to be expressed at relatively lower levels compared to GlyRa3 in PV7 RGCs. This made detection difficult by the co-localization approach that I used. 


\section{PVO RGCs Express GlyRa2 and GlyRa4 Subunits and Synaptic Currents}

The PV0 RGC is a type of ON-OFF bi-stratified RGC. Both luminance increments and decrements within its RF center evoke a transient response that is shorter than the duration of stimulus (Farrow et al., 2013; c.f. Figure 2). PV0 RGCs have medium soma diameters (Figure 5.1B) and their small dendritic fields co-localize with the On and Off ChAT bands (Figure 5.1A, 5.8A). Their morphology resembles a previously described symmetric ON-OFF direction selective RGC (DSGCs; Huberman et al., 2009; Kay et al., 2011). These RGCs are subgroup of GFP labeled cells in the TRHR mouse line (Figure 5.8B; RivlinEtzion et al., 2011). Because PV0 and TRHR RGCs appear to be morphologically similar, I did not compare their visual activity or determine their direction preference. I recorded the glycinergic sIPSCs in both $P V^{C r e}$ and TRHR lines.

In the presence of PTX and TPMPA, prominent sIPSCs remained in both WT PV0 and TRHR RGCs (Figure 5.9Aii). These sIPSC were completely blocked by strychnine (Figure 5.9Aiii). The isolated glycinergic sIPSC recorded from WT PV0 RGCs displayed a slow mean $\mathrm{T}_{\text {Decay }}(21.4 \pm 0.5 \mathrm{~ms})$, which was similar to the

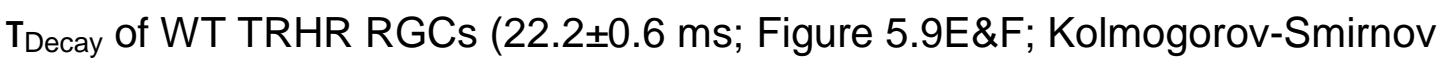
test, $p>0.05$ ). The majority ( $85 \%$ ) of glycinergic sIPSCs in both PV0 and TRHR had a $\mathrm{T}_{\text {Decay }}$ between 10 to $30 \mathrm{~ms}$ (Figure 5.9E). In addition to their morphological 
similarities, my results indicate that PVO and TRHR RGCs also receive the same glycienrgic sIPSCs.
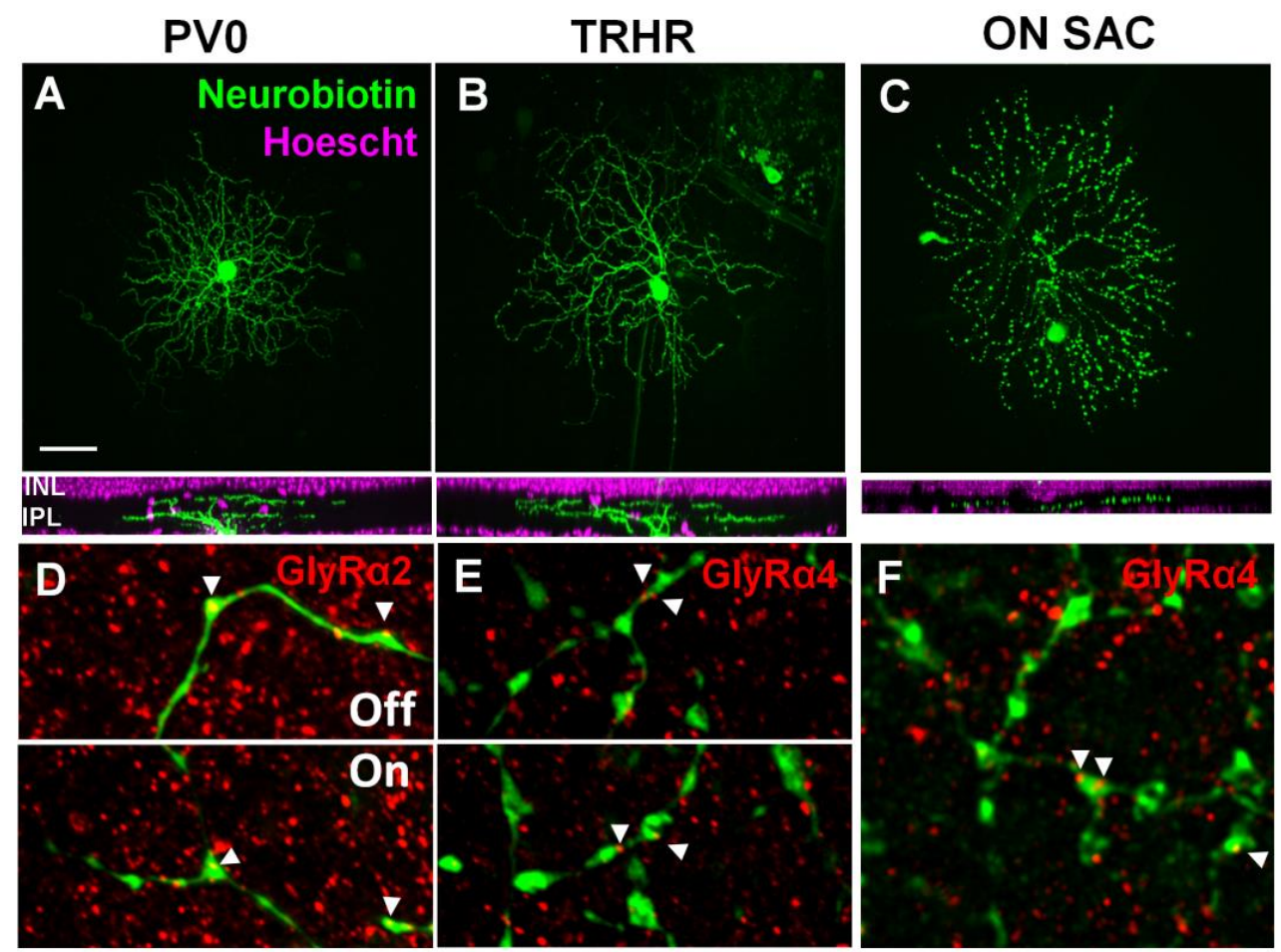

Gi

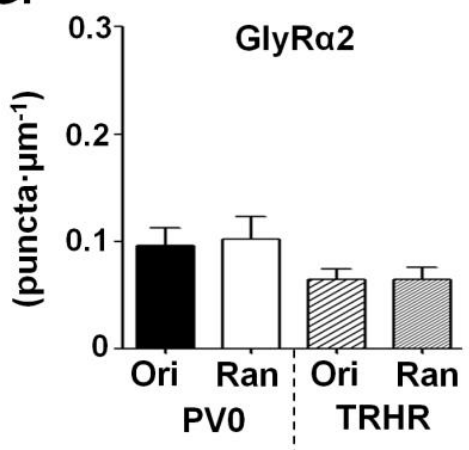

$\mathrm{Gii}$
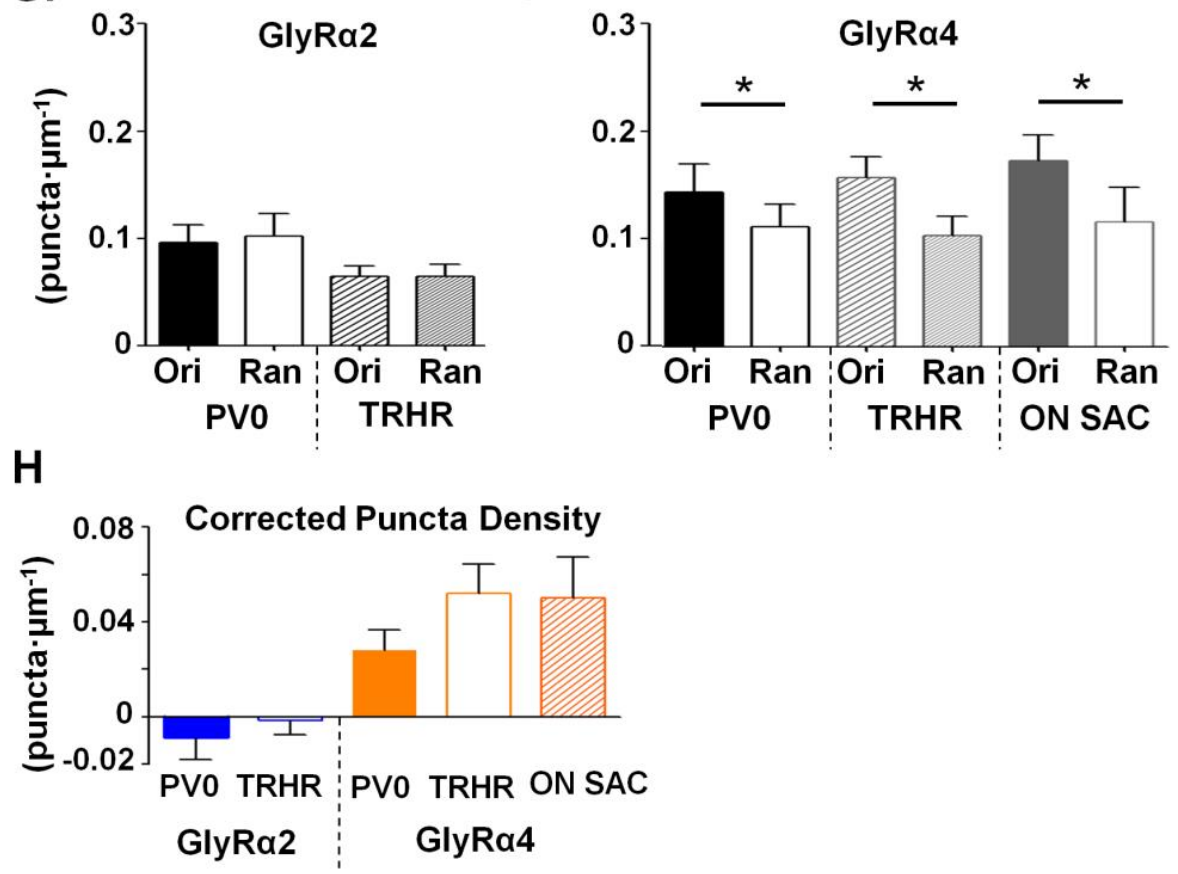
Figure 5.8 The morphology of PV0 and TRHR RGCs and expression of different GlyRa subunits on PV0 dendrites. (A-C) Top, representative en face wholemount confocal images of neurobiotin labeled (green) PV0, TRHR RGC and ON SAC ; Bottom, rotated view of the same RGCs that show the bi-stratified dendritic lamination pattern within the inner plexiform layer defined by Hoescht nuclear staining (magenta). (D-F) Representative images of double labeling of GlyRa2 (D) or GlyRa4 (E, red) with neurobiotin labeled PV0 RGC dendrites (green) in the On and Off sublamini, and GlyRa4 with ON SAC dendrites (F). The white arrows indicate subsets of co-localized GlyRa puncta on the dendrites of cells. (G) Comparisons of the original (Ori) and random density (Ran) of colocalized GlyRa2 puncta (Gi) in PV0 ( $n=6$ cells, $N=8$ areas) and TRHR ( $n=3$, $\mathrm{N}=5) \mathrm{RGCs}$, and GlyRa4 puncta (Gii) in PV0 ( $\mathrm{n}=3, \mathrm{~N}=6)$, TRHR ( $\mathrm{n}=3, \mathrm{~N}=5)$ RGCs and ON SACs $(n=2, N=3)$. (H) The corrected puncta density of GlyRa2 on the dendrites of PVO and TRHR RGCs, and GlyRa4 on the dendrites of PV0, TRHR RGCs and ON SACs. Abbreviations: INL, inner nuclear layer; IPL, inner plexiform layer. Scale bar $=40 \mu \mathrm{m}$ in A-C; $5 \mu \mathrm{m}$ in D\&E; $3 \mu \mathrm{m}$ in $\mathrm{F}$.

Because of the slow decay of their sIPSCs, either GlyRa2 or/and GlyRa4 are the likely candidates in PV0/TRHR RGCs. To determine whether PV0 and TRHR RGCs express GlyRa2 and/or GlyRa4, I recorded and characterized sIPSCs in GIra2 ${ }^{-/}$PV0 and TRHR RGCs and Glra4 ${ }^{-/}$TRHR RGCs. The absence of GlyRa2 expression in Glra2 ${ }^{-/}$PV0 and TRHR RGCs did not alter the $\mathrm{T}_{\text {Decay }}$ or 
frequency of glycinergic sIPSCs compared to WT (Figure 5.9D-F). The $\mathrm{T}_{\text {Decay }}$ of glycinergic sIPSCs was slower in the absence of GlyRa4 expression in Glra4 ${ }^{-1-}$ TRHR RGCs compared to WT (Figure 5.9F; Kolmogorov-Smirnov test, $p=0.02$ ). However, the frequency of sIPSCs were the same with WT (Figure 5.5D). This suggests that these RGCs express other GlyR a subunits. I recorded the glycinergic sIPSCs from ON SACs, which predominantly express GlyRa4mediated synaptic currents (Majumdar et al., 2009). I used the distribution of $\mathrm{T}_{\text {Decay }}$ in ON SACs to compare to the PV0 and TRHR RGCs. The mean $\mathrm{T}_{\text {Decay }}$ measured from my ON SACs glycinergic sIPSC was faster than previously reported (Majumdar et al., 2009), most likely due to the physiologically recording temperature in my study $\left(28.0 \pm 0.7 \mathrm{~ms}\right.$ at $34^{\circ} \mathrm{C}$ vs. Majumdar et al., $66.2 \pm 90 \mathrm{~ms}$ at $25^{\circ} \mathrm{C}$; Figure $\left.5.9 \mathrm{E} \& \mathrm{~F}\right)$. The $\mathrm{T}_{\text {Decay }}$ of sIPSCs in ON SACs were significantly slower compared to PV0 and TRHR RGCs and the distribution had more events $>30$ ms (Figure 5.9E; Kolmogorov-Smirnov test, both $p<0.001$ ). These results suggest that GlyRa4 contributes to the synaptic input in WT PV0 and TRHR RGCs, but is not the only GlyR a subunit responsible for glycinergic synaptic input. The other GlyRa subunit that also could be expressed is likely to be GlyRa2 and not GlyRa1 or GlyRa3, because 1) GlyRa1 or GlyRa3 mediate much faster currents (Weiss et al., 2008; mean $\mathrm{T}_{\text {Decay }} \leq 11 \mathrm{~ms}$ ) while the majority of events in WT PV0 and TRHR were around 15-20 ms (Figure 5.9E); 2) sIPSCs with slow kinetics persist in G/ra3 ${ }^{-/}$TRHR RGCs and have the same frequency and mean $\mathrm{T}_{\text {Decay }}$ compared to WT (Figure 5.9D\&F); 3) the immunostaining of 
GlyRa1 (N=2) and GlyRa3 (N=2) in PV0 and TRHR RGCs showed no significant co-cocalization (data not shown); 4) previous studies on small/wide field and displaced wide field ACs found glycinergic SIPSC with slow $\mathrm{T}_{\text {Decay }}$ and suggested expression of both GlyRa2 and GlyRa4 on these cells (Lee et al., 2015;

Majumdar et al., 2009; Veruki et al., 2007; Weiss et al., 2008).

The GlyRa2 subunit is expressed evenly throughout the IPL and GlyRa4 is mainly expressed in a band that co-localizes with ON ChAT band (Figure 1.6 \& 1.7). Immunohistochemical analysis showed significant expression of GlyRa4 puncta on both PV0 and TRHR RGC dendrites (Figure 5.8). In contrast, although some GlyRa2 immunoreactive puncta co-localize with their dendrites in both On and Off sublaminae (Figure 5.8D), the original and random coincidence rates were similar (Figure 5.8G\&H). This suggests low expression level of GlyRa2 and my co-localization analysis may not be sufficiently sensitive to show a difference. 
Ai

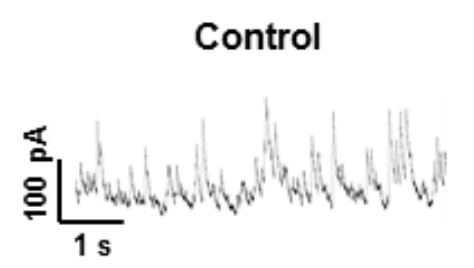

Aii

PTX+TPMPA

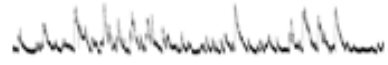

B

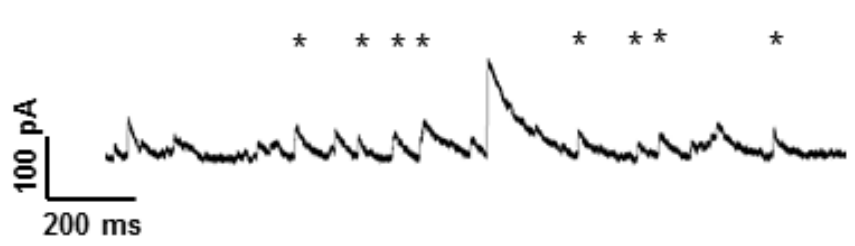

Aiii

PTX+TPMPA

+strychnine
D

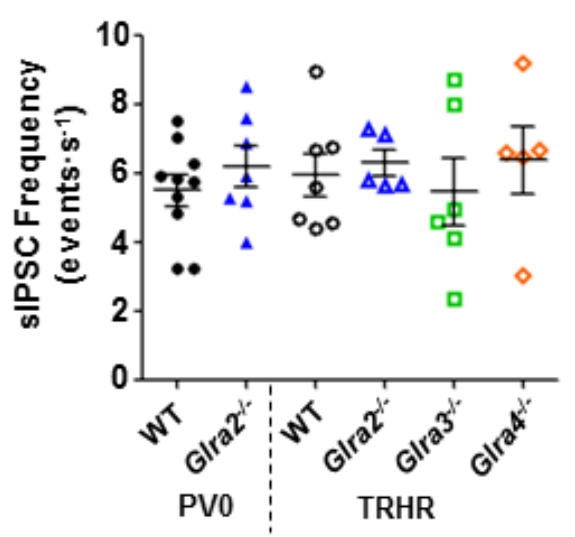

E

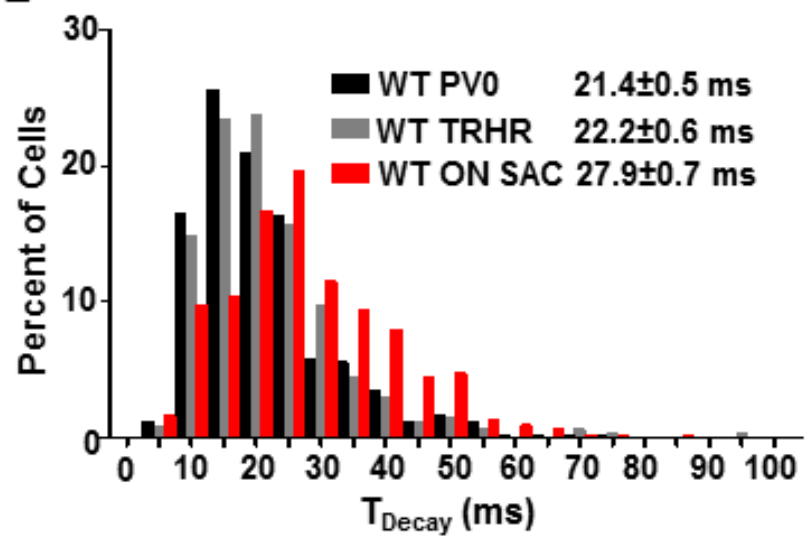

$\mathbf{F}$

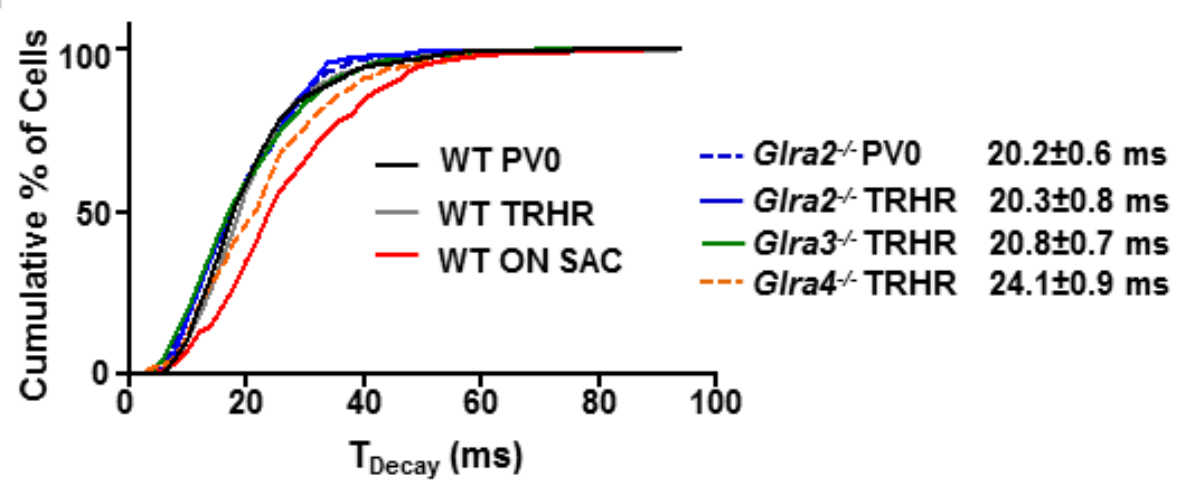

Figure 5.9 PV0 and TRHR RGCs receive sIPSC with slow decay constant. (A)

Representative sIPSCs recorded from a PVO RGC under control condition (Ai), in presence of picrotoxin (PTX, $15 \mu \mathrm{M})$ and TPMPA $(50 \mu \mathrm{M})$ without (Aii) and with (Aiii) strychnine $(10 \mu \mathrm{M})$. (B) sIPSCs recorded from the same cell in Aii at 
higher temporal resolution. Stars indicate sIPSCs that met my criterion (see methods) and were used in the analysis. (C) Average waveform of the sIPSCs from the same recording in Aii that met the criterion (90 events). The decay constant was 20.1 ms with a single exponential fitting (10-90\% of decay). The 10$90 \%$ of peak rise time was $1.8 \mathrm{~ms}$. (D) The frequency of the glycinergic sIPSCs in PV0, TRHR RGCs in WT and GlyRa knockout mouse retina. (E) The frequency distribution of the $T_{\text {Decay }}$ in sIPSCs of WT PV0 ( $n=10$ cells, $N=435$ events), TRHR ( $n=7, N=338)$ RGCs and ON SACs ( $n=8, N=444)$. (F) Cumulative frequency distribution of the $\mathrm{T}_{\text {Decay }}$ in PV0, TRHR RGCs and ON SACs in WT and GlyRa knockout mouse retina. Glra2 ${ }^{-/}$PV0, $n=7, N=322 ;$ Glra2 ${ }^{-/}$TRHR, $n=5$, $\mathrm{N}=172$; Glra3 ${ }^{-/}$TRHR, $\mathrm{n}=6, \mathrm{~N}=298$; Glra4 ${ }^{-/}$TRHR, $\mathrm{n}=5, \mathrm{~N}=195$.

\section{Discussion}

\section{Diversity in Glycinergic Synaptic Input to Morphologically Identified RGC}

\section{Types}

In the mammalian retina, subunit-specific glycinergic synaptic inputs have been observed in various classes of cells including BCs (Ivanova et al., 2006), ACs (Frech et al., 2001; Gill et al., 2006; Majumdar et al., 2009; Veruki et al., 2007; Weiss et al., 2008) and RGCs (Majumdar et al., 2007). My study extends these previous surveys across more morphologically identified RGCs and I find that all four GlyR a subunits mediate synaptic glycinergic input in subsets of 
RGCs (Table 5.2). Previous studies in rats (Protti et al., 1997) and mice (Tian et al., 1998) suggest that only half of the recorded RGCs receive glycinergic sIPSC. The difference between the previous findings and my results could be due to 1) the type of sampled RGCs might be different, although it can not be confirmed since the RGC types in previous studies were not reported; 2) the recording tempareture is different $\left(25 \mathrm{vs} .34^{\circ} \mathrm{C}\right)$ and the spontaneous release of neurotransmitter within the retina might be more active at physiological temperature in my study; 3) TPMPA was used in my study to block $G A B A_{c} R s$, which could result in more active spontaneous release of glutamate from BCs and increased downstream AC inhibitory output. Therefore, the estimated frequency of the glycinergic sIPSC in my study might not represent the actual level of spontaneous glycinergic input in the RGCs and I only compared the frequency between WT and GlyR KO mice to determine whether any specific GlyR $\alpha$ subunit contributes to the synaptic glycinergic input in RGCs. Nevertheless, my results are consistent with the study in rats where all the RGCs receive glycine-evoked IPSCs, indicating expression of GlyRs across different RGCs (Protti et al., 1997).

The GlyRa1 subunit is the most widely expressed in the central nervous system (Dutertre et al., 2012). Similarly more than half of the PV RGCs (PV1, 2, 4, 5 and 6) express GlyRa1 on their dendrites and this subunit mediates the majority of their glycinergic sIPSCs. Although GlyRa1 is expressed more densely in the Off than On IPL sublamina, the puncta density on RGC dendrites is not correlated with this expression pattern. For example, PV1 (ON) RGCs have 
similar GlyRa1 density to PV6 (OFF) RGCs. Instead the higher density of GlyRa1 in the Off sublamina is more likely related to its expression on the axon terminals of all of the OFF cone BCs.

The GlyRa3 subunit is expressed and sIPSCs with moderaly fast $\mathrm{T}_{\text {Decay }}$ are found predominantly in PV4 and PV7 (OFF) RGCs. In GlyR KO mice, the absence of its expression lowered the frequency of glycinergic sIPSC in both RGC types. The remaining sIPSCs had either faster $\mathrm{T}_{\text {Decay }}$ in PV4 RGCs or slower $_{\text {Decay }}$ in PV7 RGCs compared to WT. The decrease in frequency could be due to a decrease in presynaptic tonic glycine release and/or number of postsynaptic GlyRa3 receptors. The changes in the decay kinetics of sIPSCs indicate a change in subunit composition of the postsynaptic GlyRs.

The GlyRa4 subunit is expressed on PV0 and on TRHR RGCs. Consistent with this finding, the glycinergic sIPSCs of these RGCs had slow $\mathrm{T}_{\text {Decay }}$ and there were significant co-localization of GlyRa4 with their dendrites. My recordings suggest that GlyRa2 also could be co-expressed with GlyRa4 in PV0 and TRHR RGCs and with GlyRa3 in PV7 RGCs. This idea is not supported by my immunostaining results that said I could not demonstrate GlyRa2 co-localization with the dendrites of any of these PV RGCs. This negative result may indicate that GlyRa2 is not expressed in these RGCs, but more likely is due to the low expression level of GlyRa2 on the RGC dendrites, and/or high uniform density of GlyRa2 throughout the IPL. This results in a high random puncta coincidence rate that is difficult to distinguish from the original coincidence rate. Another possibility is that unlike the other GlyR a subunits, GlyRa2 is unevenly distributed 
across the RGC dendritic field and that makes it difficult to assess without a complete examination of the dendritic arbor.

Table 5.2 Summary of GlyR subunit-specific sIPSC properties in PV RGCs

\begin{tabular}{|c|c|c|c|c|c|}
\hline & RGC Cell Type & $\begin{array}{l}\text { Rise Time } \\
\text { (ms) }\end{array}$ & $\begin{array}{l}T_{\text {Decay }} \\
\text { (ms) }\end{array}$ & $\begin{array}{c}\text { Amplitude } \\
\text { (pA) }\end{array}$ & $\begin{array}{c}\text { GlyRa } \\
\text { Subunit }\end{array}$ \\
\hline TRHR & ON-OFF DS & $3.2 \pm 0.07$ & $22.2 \pm 0.6$ & $54.4 \pm 1.6$ & GlyRa2, a4 \\
\hline PVO & ON-OFF DS & $2.9 \pm 0.06$ & $21.4 \pm 0.5$ & $55.7 \pm 1.5$ & GlyRa2, $\alpha 4$ \\
\hline PV1 & Sustained ONa & $1.2 \pm 0.02$ & $3.0 \pm 0.1$ & $41.6 \pm 1.1$ & GlyRa1 \\
\hline PV2 & Transient ON & $1.0 \pm 0.02$ & $2.9 \pm 0.1$ & $53.4 \pm 1.9$ & GlyRa1 \\
\hline PV4 & Transient OFF & $1.6 \pm 0.03$ & $5.2 \pm 0.1$ & $33.0 \pm 0.8$ & GlyRa1, $\alpha 3$ \\
\hline PV5 & Transient OFF $\alpha$ & $1.2 \pm 0.03$ & $2.9 \pm 0.1$ & $76.8 \pm 4.7$ & GlyRa1 \\
\hline PV6 & Sustained OFFa & $1.2 \pm 0.03$ & $2.7 \pm 0.1$ & $129.3 \pm 5.9$ & GlyRa1 \\
\hline PV7 & Transient OFF & $1.6 \pm 0.02$ & $6.6 \pm 0.1$ & $32.4 \pm 0.6$ & GlyRa2, a3 \\
\hline
\end{tabular}

\section{Homomeric and Heteromeric GlyRs in RGCs}

Two types of GlyRs has been discovered in mammalian CNS: the homomeric GlyRs that contain five $\alpha$ subunits and heteromeric GlyRs that contain two $\alpha$ subunits and three $\beta$ subunits (Betz and Laube et al., 2006; Dutertre et al., 2012; Lynch et al., 2009). The glycinergic sIPSCs that I recorded are most likely mediated by heteromeric glycine receptors, because 1) 
eliminating the expression of gephyrin, a protein that binds to GlyR $\beta$ subunits to localize GlyRs on the membrane, prevents clustering of synaptic GlyRs, suggesting $\beta$ subunits are present in all the synaptic GlyRs (Feng et al., 1998; Fischer et al., 2000); 2) homomeric GlyRs that contain only $\alpha 1$, $\alpha 2$ or $\alpha 3$ subunits are much more sensitive to picrotoxin than the heteromeric GlyRs containing both $\alpha$ and $\beta$ subunits (Pribilla et al., 1992; Yang et al., 2007). With the concentration of PTX used in my study $(15 \mu \mathrm{M})$, the majority of the currents mediated by homomeric GlyRs containing ( $\alpha 1, \alpha 2$ or $\alpha 3$ ), should be completely blocked, leaving all the recorded sIPSCs mediated by heteromeric GlyRs; 3) an immunohistochemical study using GlyR subunit-specific antibodies showed that more than $90 \%$ of the GlyRa1, 2 and 3 clusters were also GlyR $\beta$-immunoreactive (Weltzien et al., 2012). In contrast, in the same analysis 50\% of the synaptically localized GlyRa4 puncta in layer 3/4 of the IPL, where the ON ChAT band is located and GlyRa4 had the highest density, was found to lack GlyR $\beta$ and gephyrin immunostaining. To date the sensitivity of homomeric a4 GlyRs to picrotoxin remains unclear. Therefore, there are likely to be both homomeric and heteromeric GlyRa4 receptors mediating synaptic currents in my study.

\section{Homogeneous and Heterogeneous Composition of GlyRa Subunit in RGCs}

In the mouse retina, some retinal cells predominantly express one type of GlyR a subunit. For example, polyaxonal amacrine-S4, wide-field amacrine (WA)-S3 and WA-multi cells express GlyRa2 (Majumdar et al., 2009). Rod BCs and all types of OFF BCs and A-type RGCs express GlyRa1 (Ivanova et al., 2006; Majumdar et al., 2007). Similarly in my study, there are PV RGCs 
predominantly expressing GlyRa1 (PV1, 2, 5, and 6). This study does not exclude the possibility of very low expression level of other GlyR a subunits in these RGCs. However, RNAi of GlyRa1 in PV5 RGCs eliminated almost all glycinergic synaptic input (Zhang et al., 2014) and knockout of GlyRa1 in immature A-type RGCs (most likely, PV1, 5 and 6) eliminated 93\% of glycinergic sIPSCs (Majumdar et al., 2007). Taken together the data suggest homogeneous expression of $\alpha 1 \beta$ receptors in these RGCs. Although PV1, 2, 5 and 6 RGCs all have large soma size (Figure 5.1B), they represent ON and OFF, sustained and transient response classes. Whether there is any correlation between RGC morphology/function and GlyR subunit-specific expression requires more investigation.

On the other hand, the composition of GlyRs is more complex in other retinal cells; some express more than one type of GlyR $\alpha$ subunit. For example, type 5, 6, 7 and A8 ACs express both GlyRa2 and GlyRa4 subunits (Lee et al., 2015; Weiss et al., 2008). Although ON SACs receive GlyRa4-predominant glycinergic synaptic input, the absence of GlyRa2 expression reduced half of their glycine-induced current, suggesting a co-expression of GlyRa2 extrasynaptically (Majumdar et al., 2009). In All ACs, the absence of GlyRa3 expression eliminated all spontaneous synaptic input but only part of glycineinduced currents, suggesting co-expression of other GlyR a subunits (Weiss et al., 2008). Similarly, some PV RGCs (PV0, 4 and 7) express multiple GlyR $\alpha$ subunits, as their glycinergic sIPSCs could not be eliminated in any single GlyR $\alpha$ subunit KO mouse. PV0 RGCs might express both GlyRa2 and GlyRa4, which is 
the same case for several types of ACs (Lee et al., 2015; Majumdar et al., 2009; Weiss et al., 2008). PV4 RGCs are likely to express both GlyRa1 and GlyRa3 given the shift to faster mean decay constants in the absence of GlyRa3 expression. PV7 RGCs seem to primarily express GlyRa3, while GlyRa2 also appears to mediate glycinergic sIPSCs.

\section{Scenarios for the Heterogeneous Composition of GlyR a Subunit in RGCs}

There are several possible scenarios for the heterogeneous composition of GlyRs in PV0, 4 and 7 RGCs. First, the RGC expresses two types of GlyRs containing different $\alpha$ subunits at different postsynaptic dendritic sites and receive different presynaptic input. Alternatively, these GlyRs could be expressed at the same postsynaptic site and receive the same presynaptic input. Finally, although not discovered in retina yet, functional recombinant GlyRs containing different $\alpha$ subunits will form in Xenopus oocytes (Kuhse et al., 1993). Therefore, there might be glycinergic channels that contain different GlyR $\alpha$ subunits expressed in RGCs, which have different decay kinetics compared to GlyRs containing single type of $\alpha$ subunit. The first scenario is the most likely in PV4 RGCs, since GlyRa1 and GlyRa3 rarely co-localized in the retina (Haverkamp et al., 2003). The second or third scenario is the more likely in PV0 and PV7, since there are many GlyRa2 receptors expressed throughout the IPL and localize close to GlyRa3 and GlyRa4 (Haverkamp et al., 2004; Heinze et al., 2007; Weiss et al., 2008).

A heterogeneous composition of GlyR $\alpha$ subunits makes it difficult to estimate receptor decay constant because it depends on not only the average, but also the proportion of each a subunit in the cell or receptors. For example, by 
using GlyR $\alpha 1-3$ subunit single $\mathrm{KO} /$ mutants, the decay constant of $\alpha 4$ containing GlyRs was estimated to be $\sim 66 \mathrm{~ms}$ in some small field ACs and ON SACs (Heinze et al., 2007; Majumdar et al., 2009). However, in several types of displaced ACs that present GlyRa2 kinetics of their sIPSCs (24ms), the absence of GlyRa2 expression did not alter the mean $\mathrm{T}_{\text {Decay }}$ in $\mathrm{Glra2}^{-/}$mice $(26 \mathrm{~ms})$, and these ACs were also hypothesized to express GlyRa4 (Majumdar et al., 2009). It was the similar case for PV0 and TRHR RGCs in my study. These evidence suggest that either 1) there are homomeric and heteromeric synaptic a 4 GlyRs that results in different kinetics of sIPSCs in different types of RGCs; or 2) GlyRa4 could be exclusively expressed in a GlyR and/or co-expressed with $\alpha 2$ within the same GlyR, creating variety in the kinetics of GlyRa4-mediated currents.

Although $\alpha 2 \beta$ GlyRs have much slower kinetics than $\alpha 3 \beta$ GlyRs (Zhang et al., 2015), the absence of GlyRa2 and GlyRa3 expression in PV7 RGCs unexpectedly resulted in same slower kinetics in the remaining sIPSCs. This result suggests that either 1) there are other GlyR a subunits that also contributes to synaptic inputs in PV7; or 2) although less likely, up-regulation of other GlyR a subunits in the absence of GlyRa2 or GlyRa3 could create complexity to estimate the GlyR composition in PV7 RGCs. Therefore, a different approach is required to solve this question. For example, AAV-RNAi could be used in adult WT mice to knockdown one or more types of GlyRs within specific groups of RGCs to isolate single type GlyR-mediated currents and avoid 
potential compensation during development, although there is little precedence in the literature for compensation in glycinergic subunits (Zhang et al., 2014).

In conclusion, the large diversity in the GlyR composition across RGC type results in various kinetics of the glycinergic synaptic inputs that indicate specific inhibitory regulation of RGC visual responses (Figure 5.10).

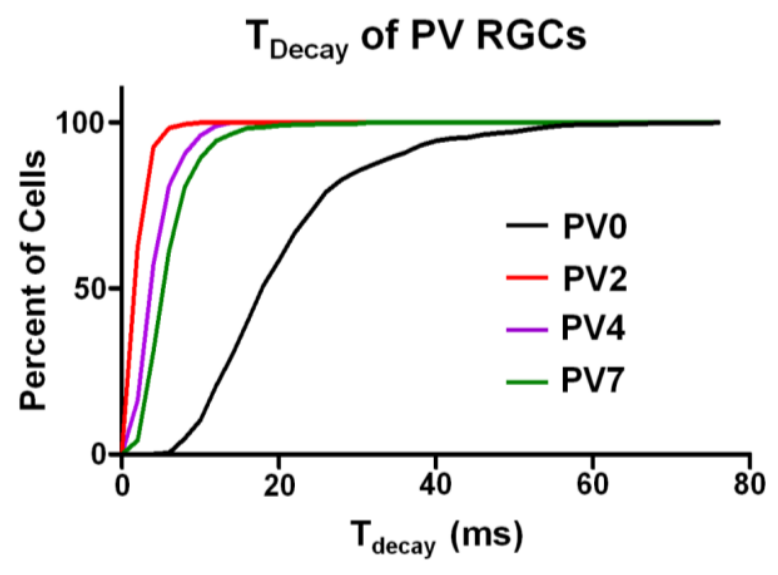

Figure 5.10. Cumulative frequency distribution of $T_{\text {decay }}$ of glycinergic sIPSCs in PV RGCs. The kinetics of tonic synaptic glycinergic input in PVO (black), PV2 (red), PV4 (purple) and PV7 (green) are different from each other (Kolmogorov-Smirnov test, all $p<0.001$ ). 


\section{CHAPTER VI \\ GENERAL DISCUSSION AND FUTURE DIRECTIONS}

Four GlyR a subunits are expressed in the mammalian CNS and together with a single type of $\beta$ subunit compose GlyRs with distinct decay kinetics (Dutertre et al., 2012; Wässle et al., 2009). The diversity in GlyRs raises the question that whether this is simply genetic redundancy or necessary for specific modulation of neuronal activities. The mouse retina expresses all four a subunits, and given our extensive knowledge of its cell classes and functional circuits this makes it the optimal model to explore this question. Overwhelming anatomical and physiological evidence are in favor of the hypothesis of subunit-specific modulation, as each of these four GlyR $\alpha$ subunits is selectively expressed across different retinal cells (Gill et al., 2006; Majumdar et al., 2007, 2009; Ivanova et al., 2006; Veruki et al., 2007; Weiss et al., 2008). What is less clear is the GlyR $\alpha$ subunit-specific inhibitory modulations at the functional level within the retinal pathways. My study addressed this question in 3 separate experiments. My results show 1) a distinct role of GlyRa1 in an identified type of RGC (Chapter IV), 2) selective roles for GlyRa2 and GlyRa3 (Chapter III) in tuning the visual activities within the On and Off pathway, and 3) a survey of the GlyR subunit expression across identified RGC types (Chapter V). 
Together with our previous study that describes the roles of GlyRa2 and GlyRa3 on RF center response properties of RGCs, my in vivo study using GlyR a subunit KO mice (Glra2 ${ }^{-/}$, Glra3 ${ }^{-/}$and Glra2/3 ${ }^{-/}$) in Chapter III showed that:

1. GlyRa2 and GlyRa3 play different roles in modulating RGC visual responses.

2. GlyRs containing GlyRa2 contribute to both the On and Off retinal pathways. In WT, GlyRa2-mediated inhibition enhances the RF center response of ON RGCs; while enhancing both the center and surround response of OFF RGCs.

3. GlyRs containing GlyRa3 contribute to only the On retinal pathway. In WT, GlyRa3-meidated inhibition enhances the RF center response of ON RGCs.

4. Different $A C$ circuits participate in GlyR subunit-specific inhibitory modulation.

These results provide strong evidence supporting the distinct functional roles of different GlyR $\alpha$ subunit, while leaving more questions that are crucial to be answered. Previously we suggested that GlyRa2 is expressed in WT OFF RGCs to mediate On-to-Off crossover inhibitory circuit that modulated the RF center responses (Nobles et al., 2014), and the RGCs are most likely to be Atype RGCs which have large axons (Boycott and Wässle, 1974; Nobles et al., 2012). However, A-type OFF RGCs predominantly express GlyRa1 (Majumdar et al., 2007; Zhang et al., 2014), not GlyRa2. There are two explanations for this discrepancy: 1) either the OFF RGCs that are under the GlyRa2-mediated 
modulation are not A-type, or 2) GlyRa2 indirectly mediates the RF center response of these OFF RGCs via other ACs, instead of a direct On-to-Off crossover circuit. One type of bistratified ACs (A8) was recently found to express GlyRa2 and make synaptic contacts with OFF BCs (Lee et al., 2015), suggesting a candidate cell to mediate On-to-Off crossover inhibition onto the OFF RGCs and receive GlyRa2-mediated inhibition which indirectly modulates RF center responses. To continue to enhance our understanding we need to identify the exact RGC types that are subject to GlyRa2/GlyRa3-mediated modulation and responsible AC circuits. We need to examine the underlying excitatory and inhibitory currents using whole cell patch clamp recording and post-recording immunohistochemistry (Chapter IV and V). The results will allow the correlation of the physiology and morphology of the RGCs. Transgenic mouse lines that have specific type of RGCs labeled by fluorescence proteins, such as PV cre (Farrow et al., 2013), could be used to further focus the study. Once the types of RGCs are defined, the recombinant G protein-deleted rabies virus could be used to retrogradely trace the presynaptic partners of the infected RGCs (Farrow et al., 2013; Wall et al., 2010). Also, the retinal connectome constructed using electron microscopy data could be explored for candidates of the proposed circuits (Anderson et al., 2011; Marc et al., 2013).

In Chapter IV, I designed and used AAV-mediated RNAi to perform subunit- and synapse-specific investigation of the role of GlyRa1 in an identified RGC type. Compared to my in vivo study using GlyR KO mouse lines, my in vitro study had following advantages: 1) it used whole cell patch clamp recording and 
targeted at specific RGCs using a reporter mouse line. This focused my study to a single type of RGCs, the OFF $\left.\alpha_{\text {Transient }} R G C s ; 2\right)$ the AAVs generate shRNAs that specifically and almost completely eliminated expression of a single type of GlyR a subunit, GlyRa1. An added benefit of this approach was that a corresponding $\mathrm{KO}$ mouse or subunit selective antagonist was not available ; 3) the AAVs were injected into dLGN to specifically infect RGCs, leaving expression of GlyRa1 intact in the rest of the retina. This localized the study to a specific synaptic level-the direct input onto the identified RGCs. With this AAV-RNAi approach, the results showed that:

1. The application of AAV-RNAi almost completely eliminated GlyRa1 expression in infected OFF $\alpha_{\text {Transient }}$ RGCs.

2. GlyRa1 is responsible for the majority of glycinergic synaptic and visually evoked inputs in OFF $\alpha_{\text {Transient }}$ RGCs, as the AAV-RNAi eliminated most of the glycinergic currents.

3. The elimination of glycinergic input not only directly demonstrated a 'push-pull' mechanism of On-to-Off crossover inhibition onto OFF RGCs, which had been suggested by several previous studies, but also revealed two feedforward GABAergic inhibitory circuits that participate in the modulation of OFF $\alpha_{T r a n s i e n t} R G C$ RF center response.

In general, the AAV-RNAi approach allows for much more thorough study of retinal circuitry, which could be applied in future work. First of all, it could be used to eliminate GlyRa1 input to other types of RGCs that significantly express 
GlyRa1 (Chapter V) to compare the role that GlyRa1 plays to shape visual responses. This can lead to a better understanding of whether there is a specific role of GlyRa1 in retinal processing. In addition, similar experiments could examine identified RGCs that express other GlyR a subunits (see Chapter V). More advanced AAV vectors could be constructed to knockdown specific subunit in other identified retinal cell classes. The AAV vectors could perform Cre-driven generation of shRNA targeting at desired genes in transgenic mouse lines where specific groups of retinal cells express Cre recombinase (Zhu et al., 2014). This could also be a follow-up project for my in vivo study to examine the effect on RGC visual activities while eliminating GlyRa2 and/or GlyRa3 expression in specific ACs that potentially modulate the responses of identified RGCs. Finally, this AAV-RNAi approach could also be used to study any other circuits of interest.

My survey in Chapter V showed the subunit-specific expression of GlyRs in different types of RGCs that could be related to specific visual function:

1. PV1, PV2, PV5 and PV6 RGCs predominately express the GlyRa1 subunit which mediates SIPSCs with fast kinetics.

2. PV0 and TRHR RGCs (DSGCs) are likely to co-express GlyRa2 and GlyRa4 which mediate sIPSCs with slow kinetics.

3. PV7 RGCs predominantly express GlyRa3 which mediate moderately fast sIPSCs.

4. PV4 RGCs are likely to co-express both GlyRa1 and GlyRa3 which create a different distribution of decay kinetics compared to other PV RGCs. 
With these data, subunit-specific AAV-RNAi (Chapter IV) could be performed to examine each type of RGC to further understand the GlyR subunitspecific modulation. Furthermore, the study suggests co-expression of two different GlyR a subunits in the same type of RGC, such as GlyRa2 and GlyRa4 in PV0/TRHR RGCs. GlyRa2 is suggested to be predominantly expressed in mammalian CNS during embryonic stage to mediate the majority of the glycinergic transmission, given the slow kinetics of synaptic glycinergic currents (Becker et al., 1988; Hoch et al., 1989; Singer et al., 1998). Surprisingly, the elimination of GlyRa2 in Glra2 ${ }^{-/}$is not lethal and the mice are phenotypically normal (Young-Pearse et al., 2006). This suggests other subunits also could be expressed in the embryo. If slow currents are required, GlyRa4 would be a good candidate and is found to be transcribed in chicken embryonic spinal cord and sympathetic nervous system (Harvey et al., 2000; Majumdar et al., 2009). Similarly, in the absence of either GlyRa2 or GlyRa4 expression, the kinetics of synaptic glycinergic currents in TRHR RGCs did not change significantly compared to WT. On the other hand, the results from my in vivo study in G/ra2 ${ }^{-1}$ show a significant change in ON and OFF RGCs visual activities, which suggest that the absence of GlyRa2-mediated inhibition cannot be simply compensated. Therefore, three questions remain to be addressed: 1) whether GlyRa4 is significantly expressed in embryonic WT and/or G/ra2 ${ }^{-/}$mouse retina; 2) if both GlyRa2 and GlyRa4 is are present in mouse embryonic retina, is this coexpression a compensation mechanism during development or 3) are they both expressed for the purpose of diversity in glycinergic modulation. 
Immunohistochemistry using GlyRa4 antibody in mouse embryonic retina could address the first question. To address the second and third questions, AAV-RNAi could be performed to knockdown the expression of GlyRa2 subunit in mature retina to examine the effect on PV0/TRHR RGCs, which avoids any possible developmental compensation in transgenic KO mice.

In summary, to have a full picture of glycinergic inhibition in the retina, many more questions need to be answered. There are approximately 30 types of ACs and 20 types of RGCs in the mammalian retina that differ in their morphology, and/or response profiles to visual stimuli (MacNeil and Masland, 1998; MacNeil et al., 1999; Masland, 2001; Lin and Masland, 2006; Sun et al., 2002; Rockhill et al., 2002; Wässle, 2004). Previous studies (Majumdar et al., 2007, 2009; Weiss et al., 2008) together with my survey (Chapter V) have only examined the GlyR subunit-specific expression in $50 \%$ of the ACs and $30 \%$ of the RGCs. Therefore, a larger scale survey needs to be performed to cover all the ACs and RGCs. To complete the puzzle of subunit-specific glycinergic circuitry, the connectivity between different ACs and RGCs needs to be examined. To understand the functional roles of these subunit-specific glycinergic circuits, genetic manipulation and electrophysiological approach needs to be combined to serve this purpose. Given there is transition of predominant subunit GlyR a subunit (GlyRa2 to GlyRa1) from embryonic/neonatal to postnatal stage in the other area in the CNS (Dutertre et al., 2012), the retina needs to be examined to determine whether it also experiences such transition and whether GlyRa3 and GlyRa4 subunit also vary in 
the expression level during the development. Whether the transition in subunits plays significant functional roles in the maturation of retinal connectivity will be intriguing question to be answered. 


\section{REFERENCES}

Anderson, J.R., Jones, B.W., Watt, C.B., Shaw, M.V., Yang, J.H., Demill, D., Lauritzen, J.S., Lin, Y., Rapp, K.D., Mastronarde, D., et al. (2011). Exploring the retinal connectome. Molecular vision 17, 355-379.

Awatramani, G.B., and Slaughter, M.M. (2000). Origin of transient and sustained responses in ganglion cells of the retina. JNeurosci 20, 7087-7095.

Azeredo da Silveira, R., and Roska, B. (2011). Cell types, circuits, computation. Current opinion in neurobiology 21, 664-671.

Badea, T.C., and Nathans, J. (2004). Quantitative analysis of neuronal morphologies in the mouse retina visualized by using a genetically directed reporter. J Comp Neurol 480, 331-351.

Beaudoin, D.L., Manookin, M.B., and Demb, J.B. (2008). Distinct expressions of contrast gain control in parallel synaptic pathways converging on a retinal ganglion cell. J Physiol 586, 5487-5502.

Becker, C.M., Hoch, W., and Betz, H. (1988). Glycine receptor heterogeneity in rat spinal cord during postnatal development. The EMBO journal 7, 37173726.

Betz, H., and Laube, B. (2006). Glycine receptors: recent insights into their structural organization and functional diversity. JNeurochem 97, 16001610.

Bloomfield, S.A., and Xin, D. (2000). Surround inhibition of mammalian All amacrine cells is generated in the proximal retina. J Physiol 523 Pt 3 , 771-783.

Borel, F., Kay, M.A., and Mueller, C. (2014). Recombinant AAV as a platform for translating the therapeutic potential of RNA interference. Molecular therapy : the journal of the American Society of Gene Therapy 22, 692701.

Borghuis, B.G., Looger, L.L., Tomita, S., and Demb, J.B. (2014). Kainate receptors mediate signaling in both transient and sustained OFF bipolar cell pathways in mouse retina. $\mathrm{J}$ Neurosci 34, 6128-6139.

Boycott, B.B., and Wässle, H. (1974). The morphological types of ganglion cells of the domestic cat's retina. J Physiol 240, 397-419.

Buffelli, M., Burgess, R.W., Feng, G., Lobe, C.G., Lichtman, J.W., and Sanes, J.R. (2003). Genetic evidence that relative synaptic efficacy biases the outcome of synaptic competition. Nature 424, 430-434.

Buldyrev, I., Puthussery, T., and Taylor, W.R. (2012). Synaptic pathways that shape the excitatory drive in an OFF retinal ganglion cell. J Neurophysiol 107, 1795-1807. 
Buldyrev, I., and Taylor, W.R. (2013). Inhibitory mechanisms that generate centre and surround properties in ON and OFF brisk-sustained ganglion cells in the rabbit retina. J Physiol 591, 303-325.

Buldyrev, I., and Taylor, W.R. (2013). Inhibitory mechanisms that generate centre and surround properties in ON and OFF brisk-sustained ganglion cells in the rabbit retina. J Physiol 591, 303-325.

Cafaro, J., and Rieke, F. (2013). Regulation of spatial selectivity by crossover inhibition. J Neurosci 33, 6310-6320.

Caldwell, J.H., and Daw, N.W. (1978). Effects of picrotoxin and strychnine on rabbit retinal ganglion cells: changes in centre surround receptive fields. J Physiol 276, 299-310.

Cascio, M. (2006). Modulating inhibitory ligand-gated ion channels. The AAPS journal 8, E353-361.

Chen, X., Hsueh, H.A., Greenberg, K., and Werblin, F.S. (2010). Three forms of spatial temporal feedforward inhibition are common to different ganglion cell types in rabbit retina. JNeurophysiol 103, 2618-2632.

Chen, X., Hsueh, H.A., and Werblin, F.S. (2011). Amacrine-to-amacrine cell inhibition: Spatiotemporal properties of GABA and glycine pathways. Vis Neurosci 28, 193-204.

Cleland, B.G., Dubin, M.W., and Levick, W.R. (1971). Sustained and transient neurones in the cat's retina and lateral geniculate nucleus. J Physiol 217, 473-496.

Cleland, B.G., Levick, W.R., and Sanderson, K.J. (1973). Properties of sustained and transient ganglion cells in the cat retina. J Physiol 228, 649-680.

Cook, P.B., and McReynolds, J.S. (1998). Modulation of sustained and transient lateral inhibitory mechanisms in the mudpuppy retina during light adaptation. JNeurophysiol 79, 197-204.

Cruz-Martin, A., El-Danaf, R.N., Osakada, F., Sriram, B., Dhande, O.S., Nguyen, P.L., Callaway, E.M., Ghosh, A., and Huberman, A.D. (2014). A dedicated circuit links direction-selective retinal ganglion cells to the primary visual cortex. Nature 507, 358-361.

Daw, N.W., Jensen, R.J., and Brunken, W.J. (1990). Rod pathways in mammalian retinae. Trends in neurosciences 13, 110-115.

Demb, J.B., and Singer, J.H. (2012). Intrinsic properties and functional circuitry of the All amacrine cell. Vis Neurosci 29, 51-60.

DeVries, S.H. (2000). Bipolar cells use kainate and AMPA receptors to filter visual information into separate channels. Neuron 28, 847-856

Dong, C.J., and Hare, W.A. (2003). Temporal modulation of scotopic visual signals by $\mathrm{A} 17$ amacrine cells in mammalian retina in vivo. $\mathrm{J}$ Neurophysiol 89, 2159-2166.

Dutertre, S., Becker, C.M., and Betz, H. (2012). Inhibitory glycine receptors: an update. The Journal of biological chemistry 287, 40216-40223.

Eggers, E.D., and Lukasiewicz, P.D. (2010). Interneuron circuits tune inhibition in retinal bipolar cells. JNeurophysiol 103, 25-37. 
Eggers, E.D., and Lukasiewicz, P.D. (2011). Multiple pathways of inhibition shape bipolar cell responses in the retina. VisNeurosci 28, 95-108.

Eggers, E.D., McCall, M.A., and Lukasiewicz, P.D. (2007). Presynaptic inhibition differentially shapes transmission in distinct circuits in the mouse retina. JPhysiol 582, 569-582.

Enroth-Cugell, C., and Robson, J.G. (1966). The contrast sensitivity of retinal ganglion cells of the cat. J Physiol 187, 517-552.

Enz, R. (2001). GABA(C) receptors: a molecular view. Biological chemistry 382, 1111-1122.

Famiglietti, E.V., Jr., and Kolb, H. (1975). A bistratified amacrine cell and synaptic cirucitry in the inner plexiform layer of the retina. Brain research 84, 293-300.

Farrow, K., Teixeira, M., Szikra, T., Viney, T.J., Balint, K., Yonehara, K., and Roska, B. (2013). Ambient illumination toggles a neuronal circuit switch in the retina and visual perception at cone threshold. Neuron 78, 325338.

Feng, G., Tintrup, H., Kirsch, J., Nichol, M.C., Kuhse, J., Betz, H., and Sanes, J.R. (1998). Dual requirement for gephyrin in glycine receptor clustering and molybdoenzyme activity. Science 282, 1321-1324.

Fischer, F., Kneussel, M., Tintrup, H., Haverkamp, S., Rauen, T., Betz, H., and Wässle, H. (2000). Reduced synaptic clustering of GABA and glycine receptors in the retina of the gephyrin null mutant mouse. J Comp Neurol 427, 634-648.

Flores-Herr, N., Protti, D.A., and Wässle, H. (2001). Synaptic currents generating the inhibitory surround of ganglion cells in the mammalian retina. $J$ Neurosci 21, 4852-4863.

Frech, M.J., Perez-Leon, J., Wässle, H., and Backus, K.H. (2001). Characterization of the spontaneous synaptic activity of amacrine cells in the mouse retina. J Neurophysiol 86, 1632-1643.

Ghosh, K.K., Bujan, S., Haverkamp, S., Feigenspan, A., and Wässle, H. (2004). Types of bipolar cells in the mouse retina. JComp Neurol 469, 70-82.

Gill, S.B., Veruki, M.L., and Hartveit, E. (2006). Functional properties of spontaneous IPSCs and glycine receptors in rod amacrine (All) cells in the rat retina. J Physiol 575, 739-759.

Greenberg, K.P., Pham, A., and Werblin, F.S. (2011). Differential targeting of optical neuromodulators to ganglion cell soma and dendrites allows dynamic control of center-surround antagonism. Neuron 69, 713-720.

Grudzinska, J., Schemm, R., Haeger, S., Nicke, A., Schmalzing, G., Betz, H., and Laube, B. (2005). The beta subunit determines the ligand binding properties of synaptic glycine receptors. Neuron 45, 727-739.

Harvey, R.J., Depner, U.B., Wässle, H., Ahmadi, S., Heindl, C., Reinold, H., Smart, T.G., Harvey, K., Schutz, B., bo-Salem, O.M., et al. (2004). GlyR alpha3: an essential target for spinal PGE2-mediated inflammatory pain sensitization. Science 304, 884-887. 
Harvey, R.J., Schmieden, V., Von Holst, A., Laube, B., Rohrer, H., and Betz, H. (2000). Glycine receptors containing the alpha4 subunit in the embryonic sympathetic nervous system, spinal cord and male genital ridge. The European journal of neuroscience 12, 994-1001.

Haverkamp, S., Muller, U., Zeilhofer, H.U., Harvey, R.J., and Wässle, H. (2004). Diversity of glycine receptors in the mouse retina: localization of the alpha2 subunit. J Comp Neurol 477, 399-411.

Hecht, S., Shlaer, S., and Pirenne, M.H. (1941). Energy at the Threshold of Vision. Science 93, 585-587.

Heinze, L., Harvey, R.J., Haverkamp, S., and Wässle, H. (2007). Diversity of glycine receptors in the mouse retina: localization of the alpha4 subunit. JComp Neurol 500, 693-707.

Helmstaedter, M., Briggman, K.L., Turaga, S.C., Jain, V., Seung, H.S., and Denk, W. (2013). Connectomic reconstruction of the inner plexiform layer in the mouse retina. Nature 500, 168-174.

Hippenmeyer, S., Vrieseling, E., Sigrist, M., Portmann, T., Laengle, C., Ladle, D.R., and Arber, S. (2005). A developmental switch in the response of DRG neurons to ETS transcription factor signaling. PLoS biology 3, e159.

Hirasawa, H., and Kaneko, A. (2003). pH changes in the invaginating synaptic cleft mediate feedback from horizontal cells to cone photoreceptors by modulating Ca2+ channels. The Journal of general physiology 122, 657671.

Hoch, W., Betz, H., and Becker, C.M. (1989). Primary cultures of mouse spinal cord express the neonatal isoform of the inhibitory glycine receptor. Neuron 3, 339-348.

Hsueh, H.A., Molnar, A., and Werblin, F.S. (2008). Amacrine-to-amacrine cell inhibition in the rabbit retina. JNeurophysiol 100, 2077-2088.

Huberman, A.D., Wei, W., Elstrott, J., Stafford, B.K., Feller, M.B., and Barres, B.A. (2009). Genetic identification of an On-Off direction-selective retinal ganglion cell subtype reveals a layer-specific subcortical map of posterior motion. Neuron 62, 327-334.

Ivanova, E., Muller, U., and Wässle, H. (2006). Characterization of the glycinergic input to bipolar cells of the mouse retina. EurJNeurosci 23, 350-364.

Kamermans, M., and Spekreijse, H. (1999). The feedback pathway from horizontal cells to cones. A mini review with a look ahead. Vision research 39, 2449-2468.

Kaneko, A., and Saito, T. (1983). Ionic mechanisms underlying the responses of off-center bipolar cells in the carp retina. II. Studies on responses evoked by transretinal current stimulation. The Journal of general physiology 81 , 603-612.

Karten, H.J., and Brecha, N. (1983). Localization of neuroactive substances in the vertebrate retina: evidence for lamination in the inner plexiform layer. Vision research 23, 1197-1205.

Kastner, D.B., and Baccus, S.A. (2013). Spatial segregation of adaptation and predictive sensitization in retinal ganglion cells. Neuron 79, 541-554. 
Kay, J.N., De la Huerta, I., Kim, I.J., Zhang, Y., Yamagata, M., Chu, M.W., Meister, M., and Sanes, J.R. (2011). Retinal ganglion cells with distinct directional preferences differ in molecular identity, structure, and central projections. J Neurosci 31, 7753-7762.

Ke, J.B., Wang, Y.V., Borghuis, B.G., Cembrowski, M.S., Riecke, H., Kath, W.L., Demb, J.B., and Singer, J.H. (2014). Adaptation to background light enables contrast coding at rod bipolar cell synapses. Neuron $81,388-$ 401.

Kemmler, R., Schultz, K., Dedek, K., Euler, T., and Schubert, T. (2014). Differential regulation of cone calcium signals by different horizontal cell feedback mechanisms in the mouse retina. J Neurosci 34, 11826-11843.

Kim, I.J., Zhang, Y., Meister, M., and Sanes, J.R. (2010). Laminar restriction of retinal ganglion cell dendrites and axons: subtype-specific developmental patterns revealed with transgenic markers. J Neurosci 30, 1452-1462.

Kim, I.J., Zhang, Y., Yamagata, M., Meister, M., and Sanes, J.R. (2008). Molecular identification of a retinal cell type that responds to upward motion. Nature 452, 478-482.

Kirby, A.W. (1979). The effect of strychnine, bicuculline, and picrotoxin on $X$ and $Y$ cells in the cat retina. The Journal of general physiology 74, 71-84.

Kirby, A.W., and Enroth-Cugell, C. (1976). The involvement of gammaaminobutyric acid in the organization of cat retinal ganglion cell receptive fields. A study with picrotoxin and bicuculline. The Journal of general physiology 68, 465-484.

Klaassen, L.J., Sun, Z., Steijaert, M.N., Bolte, P., Fahrenfort, I., Sjoerdsma, T., Klooster, J., Claassen, Y., Shields, C.R., Ten Eikelder, H.M., et al. (2011). Synaptic transmission from horizontal cells to cones is impaired by loss of connexin hemichannels. PLoS biology 9, e1001107.

Kneussel, M., Haverkamp, S., Fuhrmann, J.C., Wang, H., Wässle, H., Olsen, R.W., and Betz, H. (2000). The gamma-aminobutyric acid type A receptor (GABAAR)-associated protein GABARAP interacts with gephyrin but is not involved in receptor anchoring at the synapse. Proceedings of the National Academy of Sciences of the United States of America 97, 8594-8599.

Koike, C., Obara, T., Uriu, Y., Numata, T., Sanuki, R., Miyata, K., Koyasu, T., Ueno, S., Funabiki, K., Tani, A., et al. (2010). TRPM1 is a component of the retinal ON bipolar cell transduction channel in the mGluR6 cascade. Proceedings of the National Academy of Sciences of the United States of America 107, 332-337.

Krol, J., Busskamp, V., Markiewicz, I., Stadler, M.B., Ribi, S., Richter, J., Duebel, J., Bicker, S., Fehling, H.J., Schubeler, D., et al. (2010). Characterizing light-regulated retinal microRNAs reveals rapid turnover as a common property of neuronal microRNAs. Cell 141, 618-631.

Kuffler, S.W. (1953). Discharge patterns and functional organization of mammalian retina. JNeurophysiol 16, 37-68. 
Lee, S.C., Meyer, A., Schubert, T., Huser, L., Dedek, K., and Haverkamp, S. (2015). Morphology and connectivity of the small bistratified A8 amacrine cell in the mouse retina. $\mathrm{J}$ Comp Neurol.

Liang, Z., and Freed, M.A. (2010). The ON pathway rectifies the OFF pathway of the mammalian retina. J Neurosci 30, 5533-5543.

Lin, B., and Masland, R.H. (2006). Populations of wide-field amacrine cells in the mouse retina. J Comp Neurol 499, 797-809.

Lukasiewicz, P.D., and Werblin, F.S. (1990). The spatial distribution of excitatory and inhibitory inputs to ganglion cell dendrites in the tiger salamander retina. J Neurosci 10, 210-221.

Lukasiewicz, P.D., and Werblin, F.S. (1994). A novel GABA receptor modulates synaptic transmission from bipolar to ganglion and amacrine cells in the tiger salamander retina. J Neurosci 14, 1213-1223.

MacNeil, M.A., Heussy, J.K., Dacheux, R.F., Raviola, E., and Masland, R.H. (1999). The shapes and numbers of amacrine cells: matching of photofilled with Golgi-stained cells in the rabbit retina and comparison with other mammalian species. J Comp Neurol 413, 305-326.

MacNeil, M.A., and Masland, R.H. (1998). Extreme diversity among amacrine cells: implications for function. Neuron 20, 971-982.

Majumdar, S., Heinze, L., Haverkamp, S., Ivanova, E., and Wässle, H. (2007). Glycine receptors of A-type ganglion cells of the mouse retina. VisNeurosci 24, 471-487.

Majumdar, S., Weiss, J., and Wässle, H. (2009). Glycinergic input of widefield, displaced amacrine cells of the mouse retina. JPhysiol 587, 3831-3849.

Manookin, M.B., Beaudoin, D.L., Ernst, Z.R., Flagel, L.J., and Demb, J.B. (2008). Disinhibition combines with excitation to extend the operating range of the OFF visual pathway in daylight. JNeurosci 28, 4136-4150.

Marc, R.E., Jones, B.W., Watt, C.B., Anderson, J.R., Sigulinsky, C., and Lauritzen, S. (2013). Retinal connectomics: towards complete, accurate networks. Progress in retinal and eye research 37, 141-162.

Marc, R.E., and Liu, W. (2000). Fundamental GABAergic amacrine cell circuitries in the retina: nested feedback, concatenated inhibition, and axosomatic synapses. J Comp Neurol 425, 560-582.

Marco, S.D., Protti, D.A., and Solomon, S.G. (2013). Excitatory and inhibitory contributions to receptive fields of alpha-like retinal ganglion cells in mouse. J Neurophysiol 110, 1426-1440.

Margolis, D.J., and Detwiler, P.B. (2007). Different mechanisms generate maintained activity in ON and OFF retinal ganglion cells. J Neurosci 27, 5994-6005.

Margolis, D.J., Gartland, A.J., Euler, T., and Detwiler, P.B. (2010). Dendritic calcium signaling in ON and OFF mouse retinal ganglion cells. $J$ Neurosci 30, 7127-7138.

Masland, R.H. (2001). Neuronal diversity in the retina. Current opinion in neurobiology 11, 431-436.

Masu, M., Iwakabe, H., Tagawa, Y., Miyoshi, T., Yamashita, M., Fukuda, Y., Sasaki, H., Hiroi, K., Nakamura, Y., Shigemoto, R., et al. (1995). Specific 
deficit of the ON response in visual transmission by targeted disruption of the mGluR6 gene. Cell 80, 757-765.

McCall, M.A., Lukasiewicz, P.D., Gregg, R.G., and Peachey, N.S. (2002). Elimination of the rho1 subunit abolishes $\mathrm{GABA}(\mathrm{C})$ receptor expression and alters visual processing in the mouse retina. J Neurosci 22, 41634174.

McClements, M.E., and MacLaren, R.E. (2013). Gene therapy for retinal disease. Translational research : the journal of laboratory and clinical medicine 161, 241-254.

McClements, M.E., and MacLaren, R.E. (2013). Gene therapy for retinal disease. Translational research : the journal of laboratory and clinical medicine 161, 241-254.

Menger, N., Pow, D.V., and Wässle, H. (1998). Glycinergic amacrine cells of the rat retina. J Comp Neurol 401, 34-46.

Menger, N., and Wässle, H. (2000). Morphological and physiological properties of the A17 amacrine cell of the rat retina. Vis Neurosci 17, 769-780.

Molnar, A., Hsueh, H.A., Roska, B., and Werblin, F.S. (2009). Crossover inhibition in the retina: circuitry that compensates for nonlinear rectifying synaptic transmission. JComputNeurosci 27, 569-590.

Molnar, A., and Werblin, F. (2007). Inhibitory feedback shapes bipolar cell responses in the rabbit retina. JNeurophysiol 98, 3423-3435.

Morgans, C.W., Zhang, J., Jeffrey, B.G., Nelson, S.M., Burke, N.S., Duvoisin, R.M., and Brown, R.L. (2009). TRPM1 is required for the depolarizing light response in retinal ON-bipolar cells. Proceedings of the National Academy of Sciences of the United States of America 106, 19174-19178.

Münch, T.A., da Silveira, R.A., Siegert, S., Viney, T.J., Awatramani, G.B., and Roska, B. (2009). Approach sensitivity in the retina processed by a multifunctional neural circuit. Nature neuroscience 12, 1308-1316.

Murphy, G.J., and Rieke, F. (2006). Network variability limits stimulus-evoked spike timing precision in retinal ganglion cells. Neuron 52, 511-524.

Murphy, G.J., and Rieke, F. (2008). Signals and noise in an inhibitory interneuron diverge to control activity in nearby retinal ganglion cells. Nature neuroscience 11, 318-326.

Nelson, R., Famiglietti, E.V., Jr., and Kolb, H. (1978). Intracellular staining reveals different levels of stratification for on- and off-center ganglion cells in cat retina. J Neurophysiol 41, 472-483.

Nikolaev, A., Leung, K.M., Odermatt, B., and Lagnado, L. (2013). Synaptic mechanisms of adaptation and sensitization in the retina. Nature neuroscience 16, 934-941.

Nobles, R.D., Zhang, C., Muller, U., Betz, H., and McCall, M.A. (2012). Selective glycine receptor alpha2 subunit control of crossover inhibition between the on and off retinal pathways. J Neurosci 32, 3321-3332.

O'Brien, B.J., Richardson, R.C., and Berson, D.M. (2003). Inhibitory network properties shaping the light evoked responses of cat alpha retinal ganglion cells. VisNeurosci 20, 351-361. 
Pang, J.J., Gao, F., and Wu, S.M. (2003). Light-evoked excitatory and inhibitory synaptic inputs to ON and OFF alpha ganglion cells in the mouse retina. JNeurosci 23, 6063-6073.

Perez De Sevilla Muller, L., Shelley, J., and Weiler, R. (2007). Displaced amacrine cells of the mouse retina. J Comp Neurol 505, 177-189.

Pourcho, R.G., and Goebel, D.J. (1983). Neuronal subpopulations in cat retina which accumulate the GABA agonist, $(3 \mathrm{H})$ muscimol: a combined Golgi and autoradiographic study. J Comp Neurol 219, 25-35.

Pribilla, I., Takagi, T., Langosch, D., Bormann, J., and Betz, H. (1992). The atypical M2 segment of the beta subunit confers picrotoxinin resistance to inhibitory glycine receptor channels. The EMBO journal 11, 43054311.

Protti, D.A., Gerschenfeld, H.M., and Llano, I. (1997). GABAergic and glycinergic IPSCs in ganglion cells of rat retinal slices. J Neurosci 17, 6075-6085.

Protti, D.A., Di Marco, S., Huang, J.Y., Vonhoff, C.R., Nguyen, V., and Solomon, S.G. (2014). Inner retinal inhibition shapes the receptive field of retinal ganglion cells in primate. J Physiol 592, 49-65.

Puller, C., Ivanova, E., Euler, T., Haverkamp, S., and Schubert, T. (2013). OFF bipolar cells express distinct types of dendritic glutamate receptors in the mouse retina. Neuroscience 243, 136-148

Raviola, E., and Gilula, N.B. (1973). Gap junctions between photoreceptor cells in the vertebrate retina. Proceedings of the National Academy of Sciences of the United States of America 70, 1677-1681.

Rivlin-Etzion, M., Zhou, K., Wei, W., Elstrott, J., Nguyen, P.L., Barres, B.A., Huberman, A.D., and Feller, M.B. (2011). Transgenic mice reveal unexpected diversity of on-off direction-selective retinal ganglion cell subtypes and brain structures involved in motion processing. J Neurosci 31, 8760-8769.

Rockhill, R.L., Daly, F.J., MacNeil, M.A., Brown, S.P., and Masland, R.H. (2002). The diversity of ganglion cells in a mammalian retina. J Neurosci 22, 3831-3843.

Rodieck, R.W., and Stone, J. (1965). Analysis of receptive fields of cat retinal ganglion cells. J Neurophysiol 28, 832-849.

Roska, B., Molnar, A., and Werblin, F.S. (2006). Parallel processing in retinal ganglion cells: how integration of space-time patterns of excitation and inhibition form the spiking output. JNeurophysiol 95, 3810-3822.

Roska, B., Nemeth, E., Orzo, L., and Werblin, F.S. (2000). Three levels of lateral inhibition: A space-time study of the retina of the tiger salamander. JNeurosci 20, 1941-1951.

Russell, T.L., and Werblin, F.S. (2010). Retinal synaptic pathways underlying the response of the rabbit local edge detector. JNeurophysiol 103, 27572769.

Sagdullaev, B.T., DeMarco, P.J., and McCall, M.A. (2004). Improved contact lens electrode for corneal ERG recordings in mice. DocOphthalmol 108, 181184. 
Sagdullaev, B.T., and McCall, M.A. (2005). Stimulus size and intensity alter fundamental receptive-field properties of mouse retinal ganglion cells in vivo. VisNeurosci 22, 649-659.

Sahel, J.A., and Roska, B. (2013). Gene therapy for blindness. Annual review of neuroscience 36, 467-488.

Saito, T., and Kaneko, A. (1983). Ionic mechanisms underlying the responses of off-center bipolar cells in the carp retina. I. Studies on responses evoked by light. The Journal of general physiology 81, 589-601.

Schaefer, N., Vogel, N., and Villmann, C. (2012). Glycine receptor mutants of the mouse: what are possible routes of inhibitory compensation? Front Mol Neurosci 5, 98.

Shields, C.R., Tran, M.N., Wong, R.O., and Lukasiewicz, P.D. (2000). Distinct ionotropic GABA receptors mediate presynaptic and postsynaptic inhibition in retinal bipolar cells. J Neurosci 20, 2673-2682.

Singer, J.H., Talley, E.M., Bayliss, D.A., and Berger, A.J. (1998). Development of glycinergic synaptic transmission to rat brain stem motoneurons. J Neurophysiol 80, 2608-2620.

Snellman, J., Kaur, T., Shen, Y., and Nawy, S. (2008). Regulation of ON bipolar cell activity. Progress in retinal and eye research 27, 450-463.

Soucy, E., Wang, Y., Nirenberg, S., Nathans, J., and Meister, M. (1998). A novel signaling pathway from rod photoreceptors to ganglion cells in mammalian retina. Neuron 21, 481-493.

Sterling, P. (1995). Vision. Tuning retinal circuits. Nature 377, 676-677.

Stone, C., and Pinto, L.H. (1992). Receptive field organization of retinal ganglion cells in the spastic mutant mouse. J Physiol 456, 125-142.

Stone, C., and Pinto, L.H. (1993). Response properties of ganglion cells in the isolated mouse retina. Vis Neurosci 10, 31-39.

Strettoi, E., Dacheux, R.F., and Raviola, E. (1990). Synaptic connections of rod bipolar cells in the inner plexiform layer of the rabbit retina. J Comp Neurol 295, 449-466.

Sun, W., Li, N., and He, S. (2002). Large-scale morphological survey of mouse retinal ganglion cells. J Comp Neurol 451, 115-126.

Thibos, L.N., and Werblin, F.S. (1978). The response properties of the steady antagonistic surround in the mudpuppy retina. J Physiol 278, 79-99.

Thoreson, W.B., and Mangel, S.C. (2012). Lateral interactions in the outer retina. Progress in retinal and eye research 31, 407-441.

Tian, N., Hwang, T.N., and Copenhagen, D.R. (1998). Analysis of excitatory and inhibitory spontaneous synaptic activity in mouse retinal ganglion cells. $J$ Neurophysiol 80, 1327-1340.

Tsukamoto, Y., Morigiwa, K., Ueda, M., and Sterling, P. (2001). Microcircuits for night vision in mouse retina. J Neurosci 21, 8616-8623.

van Genderen, M.M., Bijveld, M.M., Claassen, Y.B., Florijn, R.J., Pearring, J.N., Meire, F.M., McCall, M.A., Riemslag, F.C., Gregg, R.G., Bergen, A.A., et al. (2009). Mutations in TRPM1 are a common cause of complete congenital stationary night blindness. American journal of human genetics $85,730-736$. 
van Wyk, M., Wässle, H., and Taylor, W.R. (2009). Receptive field properties of ON- and OFF-ganglion cells in the mouse retina. VisNeurosci 26, 297308.

Vaney, D.I. (1991). Many diverse types of retinal neurons show tracer coupling when injected with biocytin or Neurobiotin. Neuroscience letters 125, 187-190.

Vannier, C., and Triller, A. (1997). Biology of the postsynaptic glycine receptor. International review of cytology 176, 201-244.

Venkataramani, S., and Taylor, W.R. (2010). Orientation selectivity in rabbit retinal ganglion cells is mediated by presynaptic inhibition. J Neurosci 30 , 15664-15676.

Veruki, M.L., Gill, S.B., and Hartveit, E. (2007). Spontaneous IPSCs and glycine receptors with slow kinetics in wide-field amacrine cells in the mature rat retina. J Physiol 581, 203-219.

Volgyi, B., Chheda, S., and Bloomfield, S.A. (2009). Tracer coupling patterns of the ganglion cell subtypes in the mouse retina. J Comp Neurol 512, 664687.

Volgyi, B., Deans, M.R., Paul, D.L., and Bloomfield, S.A. (2004). Convergence and segregation of the multiple rod pathways in mammalian retina. $J$ Neurosci 24, 11182-11192.

Vroman, R., Klaassen, L.J., and Kamermans, M. (2013). Ephaptic communication in the vertebrate retina. Frontiers in human neuroscience 7, 612 .

Wall, N.R., Wickersham, I.R., Cetin, A., De La Parra, M., and Callaway, E.M. (2010). Monosynaptic circuit tracing in vivo through Cre-dependent targeting and complementation of modified rabies virus. Proceedings of the National Academy of Sciences of the United States of America 107, 21848-21853.

Wässle, H., Heinze, L., Ivanova, E., Majumdar, S., Weiss, J., Harvey, R.J., and Haverkamp, S. (2009). Glycinergic transmission in the Mammalian retina. Front MolNeurosci 2, 6.

Weiss, J., O'Sullivan, G.A., Heinze, L., Chen, H.X., Betz, H., and Wässle, H. (2008). Glycinergic input of small-field amacrine cells in the retinas of wildtype and glycine receptor deficient mice. MolCell Neurosci 37, 40-55.

Werblin, F. (1991). Synaptic connections, receptive fields, and patterns of activity in the tiger salamander retina. A simulation of patterns of activity formed at each cellular level from photoreceptors to ganglion cells [the Friendenwald lecture]. Investigative ophthalmology \& visual science 32, 459-483.

Werblin, F.S. (1974). Control of retinal sensitivity. II. Lateral interactions at the outer plexi form layer. The Journal of general physiology 63, 62-87.

Werblin, F.S. (2010). Six different roles for crossover inhibition in the retina: correcting the nonlinearities of synaptic transmission. VisNeurosci 27, 18.

Werblin, F.S. (2011). The retinal hypercircuit: a repeating synaptic interactive motif underlying visual function. J Physiol 589, 3691-3702. 
Werblin, F.S., and Dowling, J.E. (1969). Organization of the retina of the mudpuppy, Necturus maculosus. II. Intracellular recording. J Neurophysiol 32, 339-355.

Wiesel, T.N. (1959). Recording inhibition and excitation in the cat's retinal ganglion cells with intracellular electrodes. Nature 183, 264-265.

Yang, Z., Cromer, B.A., Harvey, R.J., Parker, M.W., and Lynch, J.W. (2007). A proposed structural basis for picrotoxinin and picrotin binding in the glycine receptor pore. Journal of neurochemistry 103, 580-589.

Yonehara, K., and Roska, B. (2013). Motion detection: neuronal circuit meets theory. Cell 154, 1188-1189.

Yoshida, K., Watanabe, D., Ishikane, H., Tachibana, M., Pastan, I., and Nakanishi, S. (2001). A key role of starburst amacrine cells in originating retinal directional selectivity and optokinetic eye movement. Neuron 30 , 771-780.

Young-Pearse, T.L., Ivic, L., Kriegstein, A.R., and Cepko, C.L. (2006). Characterization of mice with targeted deletion of glycine receptor alpha 2. MolCell Biol 26, 5728-5734.

Zaghloul, K.A., Boahen, K., and Demb, J.B. (2003). Different circuits for ON and OFF retinal ganglion cells cause different contrast sensitivities. J Neurosci 23, 2645-2654.

Zhang, C., and McCall, M.A. (2012). Receptor targets of amacrine cells. Vis Neurosci 29, 11-29.

Zhang, C., Rompani, S.B., Roska, B., and McCall, M.A. (2014). Adenoassociated virus-RNAi of GlyRalpha1 and characterization of its synapse-specific inhibition in OFF alpha transient retinal ganglion cells. J Neurophysiol 112, 3125-3137.

Zhang, J., Jung, C.S., and Slaughter, M.M. (1997). Serial inhibitory synapses in retina. VisNeurosci 14, 553-563.

Zhang, Y., Dixon, C.L., Keramidas, A., and Lynch, J.W. (2015). Functional reconstitution of glycinergic synapses incorporating defined glycine receptor subunit combinations. Neuropharmacology 89, 391-397.

Zhou, C., and Dacheux, R.F. (2004). All amacrine cells in the rabbit retina possess AMPA-, NMDA-, GABA-, and glycine-activated currents. Vis Neurosci 21, 181-188.

Zhu, Y., Xu, J., Hauswirth, W.W., and DeVries, S.H. (2014). Genetically targeted binary labeling of retinal neurons. J Neurosci 34, 7845-7861. 


\title{
CURRICULUM VITAE
}

\author{
Chi Zhang \\ Department of Anatomical Sciences and Neurobiology \\ University of Louisville School of Medicine \\ 301 E Muhammad Ali Blvd, Rm 407 \\ Louisville, KY 40202 \\ Phone: 502-852-0162 (lab), 502-526-3502 (home) \\ Email: valeringme@gmail.com
}

\section{EDUCATION}

University of Louisville, Louisville, KY (2008-2015)

- Ph.D. in Anatomical Sciences and Neurobiology

- Thesis: Glycine receptor alpha subunit (GlyRa) specific inhibition contributes to ganglion cell signaling in mouse retina

Xiamen University, Xiamen, Fujian, China (2002-2006)

- B. S. in Biology

- Thesis: ANT3 plays a critical role in TNF-induced cell death

\section{RESEARCH EXPERIENCE}

Graduate Research Department of Anatomical Sciences and Neurobiology, University of Louisville, KY (2010-present)

Supervisor: Maureen McCall, Ph.D.

- Examination of Glycine receptor alpha subunit-specific mediated inhibition in transgenic knockout mouse models using optic nerve recordings in vivo and whole-cell patch clamp techniques in vitro.

- Determination of protein expression in retinas using molecular biological methods.

- Performed immunohistochemistry and visualized retinal ganglion cells using confocal microscopy. 
- Maintained mouse colony, including creating new mice lineages. Graduate Research Neural Circuit Laboratories, Friedrich Miescher Institute for Biomedical Research, Switzerland (2012-2013)

Supervisors: Botond Roska, M.D., Ph.D. and Maureen McCall, Ph.D.

- Retrograde tracing of neuronal pathway of vision system using recombinant $\mathrm{AAV}$ and rabies virus.

- AAV-RNAi of specific Glycine receptor alpha subunit and functional study of subunit-specific circuit.

- Two-photon calcium imaging of retinal ganglion cell visual activities.

Graduate Research Department of Anatomical Sciences and Neurobiology, University of Louisville, KY (2008-2010)

Supervisor: Robert Lundy, Ph.D.

- Examination of neural circuits responsible for central taste processing using retrograde/anterograde tracers and confocal microscopy.

- Construction of adeno- and lenti-virus vectors designed to selectively express reporter genes in taste-related neurons.

Research Assistant Department of Biochemistry and Molecular Biology, Xiamen University, Xiamen, Fujian, China (2006-2008)

Supervisors: Jiahuai Han, Ph.D. and Huizhe Huang, Ph.D.

- Examination of genes related to TNF-induced cell death using molecular and cell biological methods.

- Construction of plasmids designed to selectively express reporter genes in gap junctions of zebrafish.

- Performed micro-injection of nucleic acids in zebrafish embryo.

\section{TEACHING EXPERIENCE}

Teaching Assistant Department of Anatomical Sciences and Neurobiology, University of Louisville, KY (2010-2011)

- Neural anatomy lab for medical students: demo presentation and preparation of exams.

\section{AWARDS AND GRANT}

First Place Winner, Graduate Students Poster Competition at Society for Neuroscience, Louisville Chapter (April 2014) 
- Poster title: "AAV-RNAi Characterization of Subunit- and Synapsespecific Glycinergic Inhibition in A-type OFF RGCs."

\section{European Molecular Biology Organization (EMBO) Short Term Fellowship (Oct 2012) \\ - Proposal title: "Define the local circuit of identified Grik4-cre retinal ganglion cells in cultured mouse retina."}

Sigma Xi Grant-in-Aid of Research (Jan 2012)

- Proposal title: "Glycine receptor alpha 2 subunit specific inhibition in mouse retina".

\section{PUBLICATIONS}

Zhang, C., Rompani, S.B., Roska, B. and McCall, M.A. "Adeno-Associated Virus (AAV)-RNAi of GlyRa1 and Characterization of Its Synapse-specific Inhibition in OFF alpha Transient Retinal Ganglion Cells." Journal of Neurophysiol. 2014 Dec 15;112(12):3125-37.

Nobles, R.D., Zhang, C., Müller, U., Betz, H. and McCall, M.A. "Selective Glycine Receptor a2 Subunit Control of Crossover Inhibition between the On and Off Retinal Pathways." Journal of Neuroscience. 2012 Mar 7; 32(10): 3321-32. Zhang, C. and McCall, M.A. "Receptor targets of amacrine cells." Visual Neuroscience. 2012 Jan; 29(1): 11-29.

Zhang, C., Kang, Y., Lundy, R.F. "Terminal field specificity of forebrain efferent axons to the pontine parabrachial nucleus and medullary reticular formation." Brain Research 2011 Jan 12; 1368: 108-18.

\section{PRESENTATIONS}

Zhang, C. and McCall, M.A. "Subunit specific expression of glycine receptor in mouse retinal ganglion cells." Poster Presentation at Society for Neuroscience in Washington, DC (Nov 2014).

Zhang, C., Rompani, S.B., Roska, B. and McCall, M.A. "Subunit specific expression of glycine receptor in mouse retinal ganglion cells." Poster Presentation at Federation of American Societies for Experimental Biology (FASEB) in Saxtons River, VT (June 2014).

Zhang, C., Nobles, R.D., and McCall, M.A. "Distinct Roles for Glycine Receptor Subunits $\alpha 2$ and $\alpha 3$ in Retinal Signaling under Light- and Dark-adapted Conditions." Poster Presentation at Federation of American Societies for Experimental Biology (FASEB) in Steamboat Springs, CO (June 2012). 
Zhang, C., Nobles, R.D., Schmid, H. and McCall, M.A. "A Shared role for glycine receptor subunits $\alpha 2$ and $\alpha 3$ in retinal On- but not Off-pathway signaling." Poster Presentation at Association for Research in Vision and Ophthamology (ARVO) in Fort Lauderdale, FL (May 2011). 